\title{
ANZSSA Heads of Counselling Services \\ HOCS Benchmarking Survey 2018 Summary Report
}

Annie Andrews

\begin{abstract}
The ANZSSA Heads of Counselling Services Benchmarking Survey 2018 is the third survey of the Heads of Counselling Services (HOCS) conducted for and by University Counselling Service Managers in collaboration with the Australian and New Zealand Student Services Association Pty Ltd (ANZSSA ww.anzssa.org). This report summarises the 2018 HOCS survey. The primary aims of the survey are to explore benchmarking and identify emerging issues related to counselling services in the post-secondary and higher education sectors by comparing: staffing, administrative processes, service delivery activities, student service users, data generated by the service, and data pertaining to the institution in which each service operates.
\end{abstract}

\section{Introduction}

During 2017 those employed in a role that served as a 'head of the counselling service' (HOCS) within universities located within Australia and New Zealand were contacted via email to construct a list of current HOCS. Subsequently, managers of counselling services in post-secondary education in Australia and New Zealand were invited to attend a HOCS conference and planning meeting held over two days at UNSW Sydney, in September 2017.

During the HOCS meeting those assembled discussed the changes, similarities and differences that are evident between university counselling services and the impact on service delivery models. The HOCS Benchmarking Survey conducted in 2013 (Andrews, 2016) was discussed and all were in agreement that the data was now outdated. The assembled HOCS requested that the HOCS Benchmarking Survey be conducted again as early as possible in 2018.

The ANZSSA Heads of Counselling Services Benchmarking Survey 2018 is the third survey of the Heads of Counselling Services (HOCS) conducted for and by University Counselling Service Managers in collaboration with the Australian and New Zealand Student Services Association Pty Ltd (ANZSSA ww.anzssa.org). Earlier HOCS benchmarking surveys were conducted in 2010 and 2013 with reports published in JANZSSA.

The primary aims of the HOCS Benchmarking survey are to explore the similarities and differences, report on best practice and to identify emerging issues and initiatives being undertaken across counselling services in post-secondary education. The survey aims to benchmark staffing and service delivery approaches and identify emerging issues related to counselling services in the post-secondary and higher education sectors. The survey gathers data available to the HOCS and explores: staffing, administrative processes, service delivery activities, student service users, data generated by the service, and data pertaining to the institution in which each service operates.

\section{Collaborative partnership}

The 2018 HOCS Benchmarking survey was undertaken as a collaborative partnership with contributions from: Gerard Hofmann (Manager Student Academic Services (Counselling) Victoria University of Wellington, New Zealand, and Convenor - ANZSSA Counsellors Special Interest Group - New Zealand), Jeremy Cass (Manager, RMIT Counselling Service, RMIT University, Victoria, Australia and Convenor, ANZSSA Counsellors Special Interest Group, Australia), Dr Sean Murray (Head of Service, Counselling, Disability and Wellbeing Services, Curtin University, Perth, Western Australia) and Annie Andrews (Director, Counselling and Psychological Services, UNSW Sydney, Sydney, Australia, ANZSSA Fellow and Co-editor, JANZSSA). 


\section{Funding}

This benchmarking exercise was not funded via any grant. The survey construction, distribution, data analysis, report writing and report distribution was supported by pro bono services provided by the lead author, with support from collaborative team colleagues as named. Some 'in kind' support was provided by several universities namely: UNSW Sydney, RMIT and Curtin University and ANZSSA the peak body for student support services in Australia and New Zealand.

\section{Survey Instrument used}

The survey was conducted online using an online survey tool (Survey Monkey) used for both prior surveys. The 2018 survey contained 142 questions. The 2018 HOCS Benchmarking Survey was deployed early in 2018 and responders were invited to return their completed surveys prior to 29 March. The date was subsequently extended until end of May 2018. Questions used in the 2018 survey were primarily based on service and institution data collected during 2017 making the surveys easily comparable.

Survey sections:

Questions 1 to 10: Questions 11 to 18 :

Counselling Service manager information

Compensation for additional hours worked and additional hours worked; services reporting to role and functions of those services; proportion of role devoted to service delivery management, clinical supervision, clinical services etc; deputy to role.

Questions 19 to 21: Budget and funding.

Questions 22 to 36: $\quad$ Professional and administrative roles including staff to student ratio and additional services provided by institution that offer student support.

Questions 37 to 56: Information about the institution, enrolment numbers, policies etc.

Questions 57 to 76: $\quad$ Service activity information.

Questions 77 to 78: Use of 'wait' lists or 'unmet demand' lists.

Questions 79 to 81: Use of screening tools.

Questions 82 to 84: Use of telephone and VOIP for counselling service delivery, after hours service provision and $24 / 7$ service provision.

Questions 85 to 90: $\quad$ Strategies used to maximise service delivery.

Questions 91 to 93: Therapeutic alliances, use of outcome measures, use of pre/post assessment/screening tools.

Questions 94 to 97: Use of consumer reference group, methods used to seek feedback and engage service users.

Questions 98 to 99: $\quad$ Service research.

Questions 100 to 103: Fees and service charges.

Questions 104 to 107: Government subsidised mental health services (Medicare etc) Australia only.

Questions 108 to 111: Services provided under MOU.

Questions 112 to 139: Counselling Service activity data.

Questions 140 to 141: Data about students who access counselling services.

Question 142:

Survey feedback.

\section{Survey distribution}

To establish the distribution channels, existing networks, including the HOCS e-list serve, the list of participants who attended the HOCS Conference held at UNSW Sydney in September 2017, and the ANZSSA member networks were utilised to ensure that the survey reached as many heads of counselling services as possible. Further efforts were made to obtain email contact details for any recently appointed HOCS by directly telephoning all university counselling services without an identified contact person. The HOCS e-list was then updated and became the primary distribution 
mechanism for the HOCS Benchmarking Survey. Surveys were sent to members HOCS list serve linking HOCS across the Australian and New Zealand post-secondary sector. Any bounced emails were then identified and efforts were made to distribute to an appropriate alternative at the identified institution. HOCS receiving the email with the information about the survey were also invited to forward to any HOCS colleague who they thought might not be aware of the survey.

To reach HOCS employed at universities and polytechnic colleges in New Zealand the survey invitation and link was sent personally by the ANZSSA Counselling Professional Interest Group coordinator for New Zealand so as to ensure that the local established network was utilised effectively and all NZ HOCS were invited to respond to the survey.

Reminders to complete the survey were sent via the primary distribution e-list and a notice of the extended dead line was also communicated. Any HOCS requesting extra time was assured that the survey would not be closed until they had submitted all their available data. Because of the length of the survey instructions were issued to print the PDF of the survey questions supplied via the email, gather the data and then complete the survey responses. HOCS were instructed that provided the survey questions were answered from the same IP address the survey responses could be paused and returned to at a later time to complete.

\section{Ethics approval}

The survey is a benchmarking exercise, conducted periodically and was instigated at the request of members of ANZSSA who manage a counselling service. Participation in the survey was by invitation only and the survey was completed on a voluntary basis. All data, and the analysis of the data, was de-identified prior to analysing, reporting and publishing. Because of these factors institutional ethics approval was not sought for this survey. It is recommended that any subsequent HOCS Benchmarking Survey be deployed with 'ethics approval'.

\section{Responses}

Australia hosts forty-three (43) universities ${ }^{1}$ and New Zealand offers thirty-six (36) universities ${ }^{2}$ and colleges. In total HOCS from thirty-eight (38) institutions responded being comprised of twenty-nine (29) Australian institutions and seven (7) New Zealand institutions and two (2) HOCS from institutions identified as located in both Australia and New Zealand. Two Australian and one New Zealand based responder/s began the survey twice, yielding twenty-seven (27) responders from Australia and six (6) responders from New Zealand. Thus the over all response was forty-two percent $(42 \%)$ if it is assumed that the survey reached all institutions. However, it is unlikely that the reach was that successful. It is more probable that the survey reached thirty-nine (39) Australian universities plus two (2) vocational education colleges and eight (8) universities in New Zealand. Based on the likely reach of the survey the overall return rate was more probably closer to sixtyseven percent $(67 \%)$.

\section{Questions and responses provided in order of the questions appearance on the survey}

Not all responders provided data for every question. Therefore, the number of responses received varied per question.

\section{Q1 Country in which the participating institution is located}

Prior to accounting for duplicate entries responses were received from thirty-eight universities (38). The responders included Australian universities (29-2 duplicate entries) and New Zealand universities ( $7-1$ duplicate entry). Two (2) universities identified as being both Australian and New Zealand based.

\footnotetext{
${ }^{1}$ https://www.studyinaustralia.gov.au/english/australian-education/universities-and-higher-education/list-of-australianuniversities

${ }^{2}$ https://www.university-list.net/New-Zealand/universities-1000.htm
} 


\section{Q2 Name and contact details of the person completing the survey}

Name and contact details of the person completing the survey has been withheld.

The participating institutions (alphabetical order): Auckland University of Technology; Australian National University; Canberra Institute of Technology; Central Queensland University; Charles Darwin University; Curtin University; Flinders University; Edith Cowan University; Federation University Australia; James Cook University; La Trobe University; Lincoln University; Macquarie University; Massey University (Auckland); Monash University; RMIT UNIVERSITY; Swinburne University; TAFE NSW; The University of Melbourne; University of Adelaide; University of Auckland; University of Newcastle; University of New England; University of Notre Dame Fremantle; University of New South Wales Canberra; University of Queensland; University of South Australia; University of Southern Queensland; University of Sydney; University of Tasmania; UNSW Sydney; Victoria University; Victoria University of Wellington; University of Western Australia.

\section{Q3 Respondent - Years Employed in Sector}

The majority of survey responders had been employed within the post-secondary/higher education sector for at least 6 years or more $(71 \%)$. These managers identified employment within the sector as: $6-10$ years $(18.41 \%) ; 11-15$ years $(18.41 \%) ; 15-120$ years $(18.41 \%) ;>20$ years $(15.78 \%)$. A number of managers $(\mathrm{n}=11)$ reported being employed within the sector within the last 4 years. Twenty-nine percent (28.93\%) reported being employed in the sector for $0-5$ years.

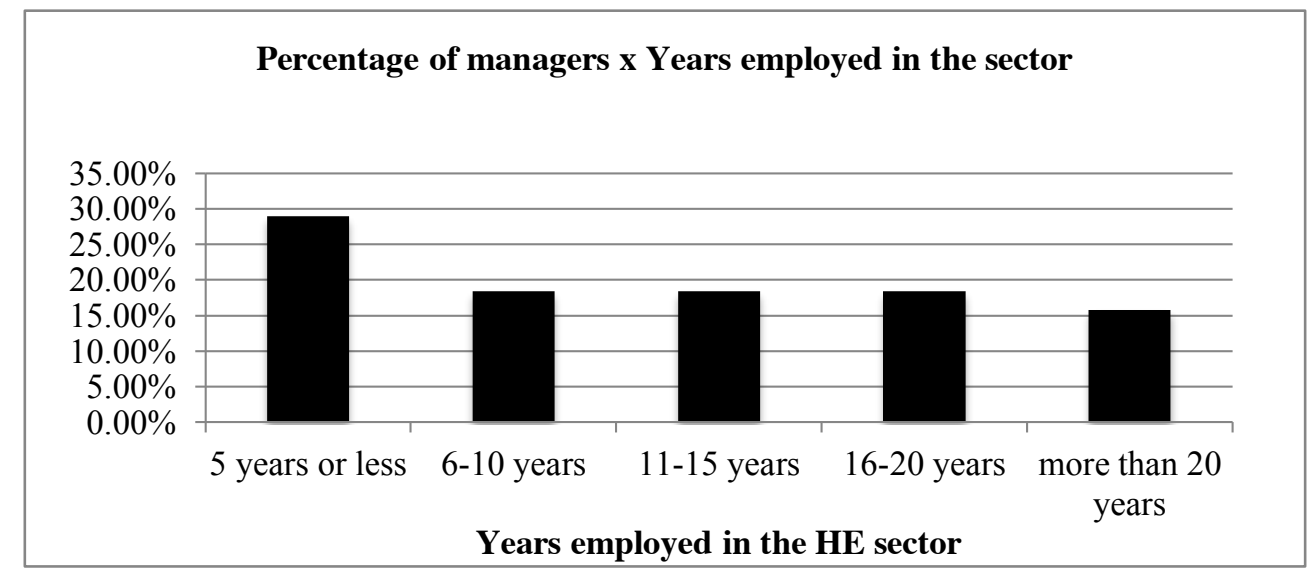

Figure 1

\section{Q4 Name of portfolio/service}

There was considerable variation within familiar themes. Counselling and Psychological Services was the most popular. The word cloud below provides a flavour of the words used within portfolio naming.

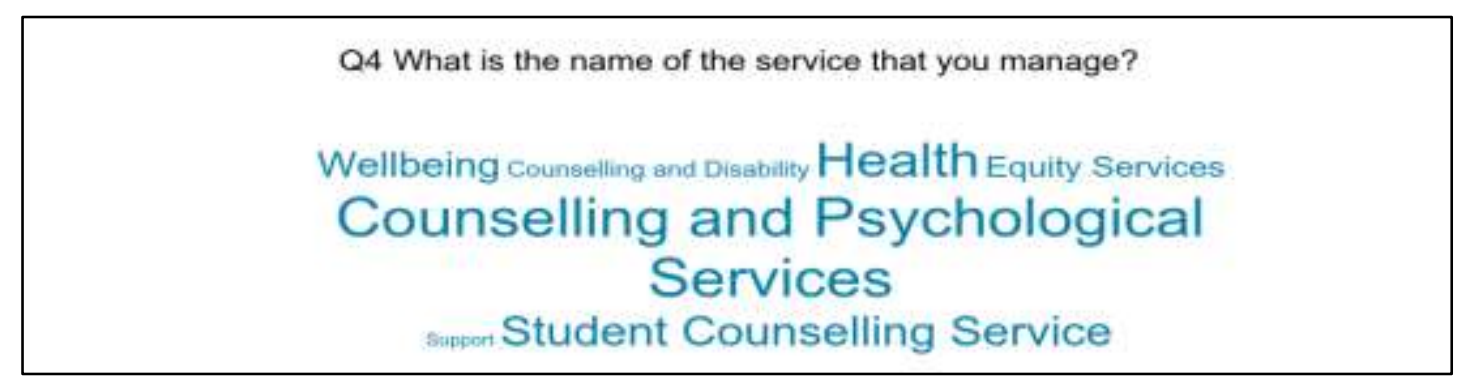

Q5 Responders professional role

- Counselling Service Manager (some counselling/clinical role) (41\%). 
- Counselling Service Manager (some counselling/clinical role) plus manager of one or more other service areas $(28.2 \%)$.

- Counselling Service Manager (no counselling/clinical role) plus manager of one or more other service areas $(10.25 \%)$.

- Other specified (10.25\%).

- Counselling Service Manager (no counselling/clinical role) $(5.12 \%)$.

- Senior Counsellor (administrative plus counselling/clinical role) (5.12\%).

\section{Q6 Manager Professional background}

Counselling Service managers were: psychologists (63\%), social workers (18.4\%), university administrators (5.2\%), the category 'other' identified as: 'registered counsellor; counsellor; mediator/conflict resolution communications $(7.89 \%)$, medical practitioner $(2.6 \%)$ and nurse $(2.6 \%)$.

$Q 7 \& Q 8$ Does the role require postgraduate qualifications and professional registration

The majority of counselling service manager positions required postgraduate qualifications (78.95\%) and professional registration $(81.58 \%)$.

Q9 Extra hours worked beyond (employment) agreement to satisfy the demands of the role

The majority of managers indicated that they work hours beyond those required by their (employment) agreement in order to satisfy the demands of their role (86.84\%).

Few managers reported not working additional hours (13.15\%).

Q10 A manager/senior counsellor delegated the responsibility to be contactable for 24/7 for emergency/crisis situations or for emergency support of students

The majority of institutions reported that a manager/senior counsellor was delegated the responsibility to be contactable $24 / 7$ for emergency/crisis situations or for emergency support of students $(60.5 \%)$.

The remaining institutions did not provide a manager/senior counsellor to be contactable 24/7 for emergency/crisis situations or for emergency support of students (39.5\%).

Q11 Pay or time-in-lieu for additional hours worked

More than half of the managers were not able to claim payment or time-in lieu for additional hours worked $(66.67 \%)$.

Only $33.33 \%$ of managers reported they could claim payment or time-in-lieu for additional hours worked.

Q12 Average hours of overtime worked

Slightly less than half (48.48\%) of the respondents worked 5 hours or less of 'overtime' per week. A similar percentage worked 6-10 additional hours per week (42.42\%) and less than 10\% worked and additional 11 hours or more on average (9.09\%). 


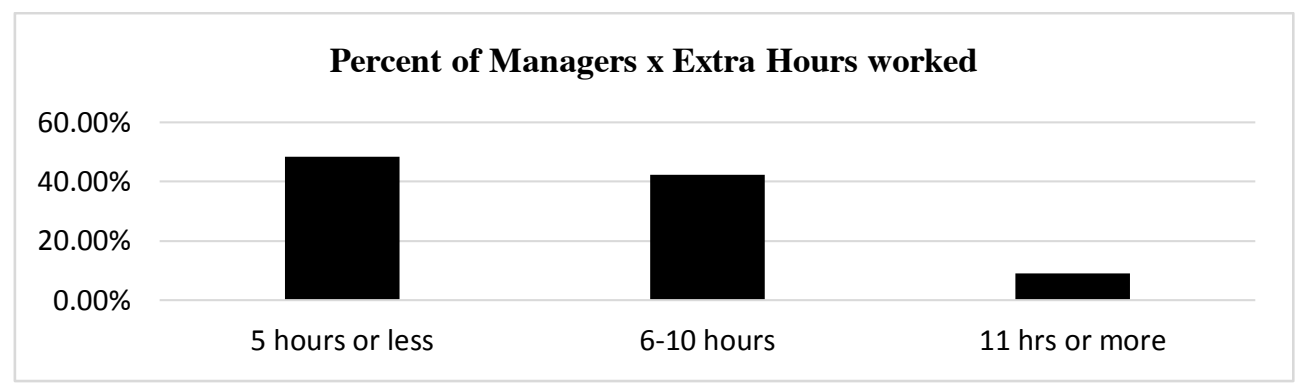

Figure 2

Q13 Number of additional services/units reporting included in managers portfolio

A number of managers $(n=17)$ focused solely on the counselling service (44.74\%). Managers were most often responsible for up to two other services in addition to the counselling service: one additional service (21\%); 2 or 3 additional services (26.3\%) and 4 additional services $(5.3 \%)$; one manager held responsibility for more than 5 services.

Q14 Functions of additional services/units in manager's portfolio

The names of additional services reported as being within in the manager's portfolio give an indication of the types of student services associated via line management with counselling services.

The HOCS portfolios were varied and for those with more than one additional service, included combinations of the following grouped services:

- Administrator/Receptionists service across at least two services

- Administration Counsellor and training coordinator

- Careers and Employment

- Disability Support Service

- National Disability Coordination Officer and ADCET Coordinator Officer

- AccessAbility Services

- Equity Services

- Equity, Diversity and Disability Service

- Student Equal Opportunity

- Equity Contact Officers Network

- LGBTI Ally program

- Health service (GPs and Nurses)

- Triage / Emergency Care (24/7 response to emergency incidents)

- Halls of Residence Wellbeing Advisors

- Student Living Health \& Wellbeing Coordinator (based in residences)

- Off-Campus Accommodation

- Health Promotion team

- Chaplaincy Program

- Multi-faith Centre 
- International (student) pastoral support

- Wellbeing Services

- Student support coordinators team

- Complaints and Conflict Resolution Support

- Student Financial Support Officer

- Financial literacy and material aid

- Sexual Assault and Harassment project officer

- Mentor Program

- Student Engagement/FUSA - student association

Q15 Proportion of manager's role focused on service delivery management

Twenty-one managers reported the proportion of their FTE used for service management was 0.6 1 FTE.

FTE 0.6 and FTE 0.8 were the two categories with the most responses gaining seven (7) (i.e. $33.33 \%$ ) and nine (9) (i.e. $42.85 \%$ ) responses respectively.

Table 1

\begin{tabular}{|c|c|}
\hline $\begin{array}{c}\text { Estimated FTE used for management } \\
\text { of service delivery }\end{array}$ & Number of Institutions \\
\hline 0.1 & 1 \\
\hline 0.2 & 2 \\
\hline 0.3 & 4 \\
\hline 0.4 & 5 \\
\hline 0.5 & 3 \\
\hline 0.6 & 7 \\
\hline 0.65 & 1 \\
\hline 0.7 & 1 \\
\hline 0.75 & 1 \\
\hline 0.8 & 9 \\
\hline 0.9 & 1 \\
\hline 1 & 1 \\
\hline
\end{tabular}

\section{Number of Institutions x FTE utilised for service management}

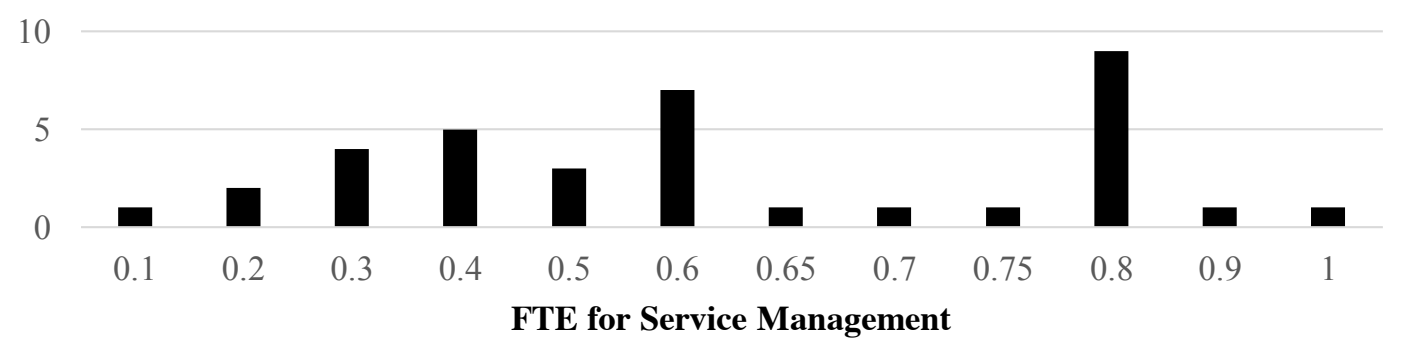

Figure 3

Q16 The proportion of manager's FTE used for clinical supervision

FTE focused on the provision of Clinical supervision was provided by all managers except for $\mathrm{N}=4$. 
The FTE allocated by the majority of respondents $(62.16 \%)$ was FTE $0.1(\mathrm{~N}=12)$ or FTE 0.2 $(\mathrm{N}=11)$.

\section{Table 2}

\begin{tabular}{|c|c|}
\hline FTE Clinical Supervision & Number of Institutions \\
\hline 0 & 4 \\
\hline 0.1 & 12 \\
\hline 0.2 & 10 \\
\hline 0.3 & 5 \\
\hline 0.4 & 11 \\
\hline 0.5 & 3 \\
\hline 0.6 & 1 \\
\hline
\end{tabular}

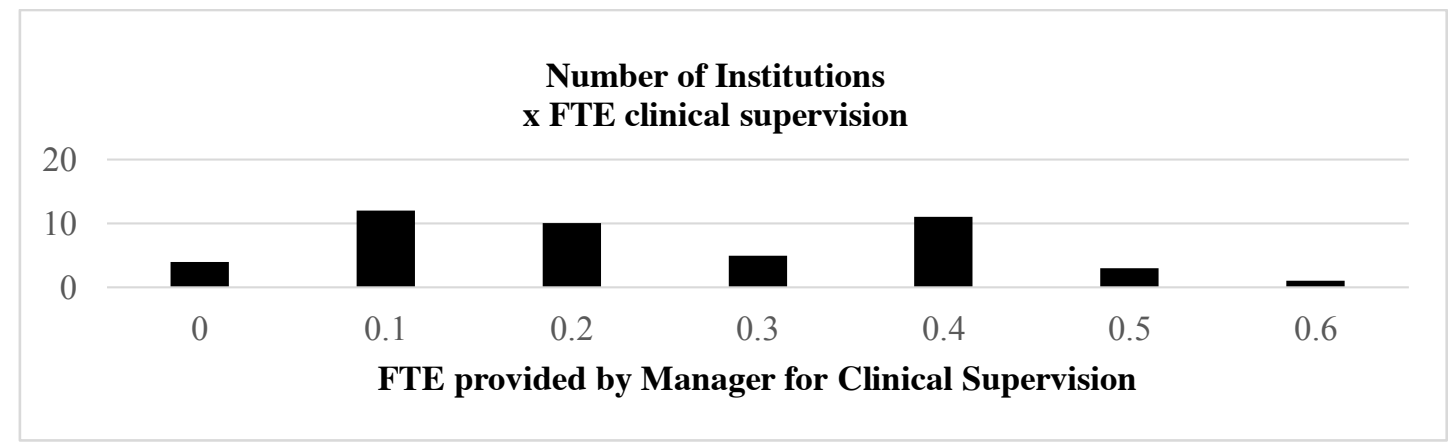

Figure 4

Q17 Proportion of the manager's FTE allocated to direct service delivery such as counselling students or facilitating workshops or preparing online resources etc

Four (4) managers did not provide FTE for direct service delivery (counselling students or facilitating workshops or preparing online resources).

Eleven (11) managers (28.94\%) dedicated FTE 0.4 of their role FTE to direct service delivery.

Table 3

\begin{tabular}{|c|c|}
\hline Manager FTE Direct Service Delivery & Number of Institutions \\
\hline 0 & 4 \\
\hline 0.1 & 8 \\
\hline 0.2 & 5 \\
\hline 0.25 & 1 \\
\hline 0.3 & 6 \\
\hline 0.4 & 11 \\
\hline 0.6 & 1 \\
\hline 0.7 & 1 \\
\hline 0.8 & 1 \\
\hline
\end{tabular}




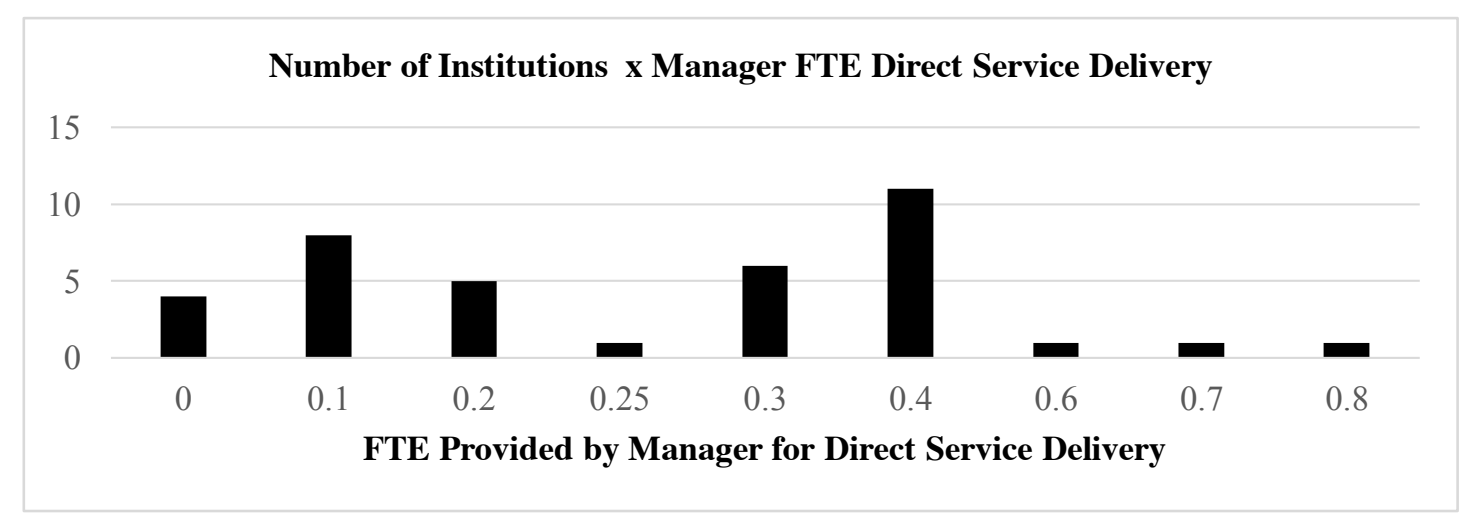

Figure 5

Q18 Deputy leader identified as support for head of service

Just over half of the mangers (18) had a member of staff who functioned as a deputy manager $(52.63 \%)$.

The remainder (47.37\%) reported that they did not have a staff member who acted as a deputy.

Q19 Source of operating funds for the counselling service

During 2017 Counselling Service financial resources were primarily tied to the institution's operating funds.

More than sixty percent $(60.53 \%)$ of survey respondents reported: Service budget allocated as part of the institution's operating budget allocation - different sources of funding for the institution are not apparent at this level.

Almost forty percent (39.47\%) of survey respondents reported: Budget supported by a compulsory student services/amenities fees paid at enrolment.

Over thirty percent $(31.58 \%)$ of survey respondents reported: Rolling allocation based on previous year's budget (may have adjustment up or down by \% imposed on all budget areas).

Over thirteen percent (13.16\%) of survey respondents indicated: Government Funds for targeted programs to provided services to students in equity groups have been made available for counselling service activities.

More than ten percent $(10.53 \%)$ of survey respondents indicated: budget supported by a voluntary student services/amenities fees paid at enrolment; budget allocation based on yearly submissions that are competitive with other services.

Counselling Service resourcing was linked to: None of the above identified funding sources.

Comments about sources of budget listed below demonstrate the, at times, opaque and the frequent complexity of funding models used for counselling services in post-secondary education:

- In the counselling and wellbeing space, funding is as follows: - Operating costs of unit - as part of institution's operating budget allocation - 2FTE Psychologists - as part of institution's operating budget allocation - 1 FTE Psychologist - Higher Education Participation and Partnership Program (HEPPP) - 2 FTE Student Wellbeing Counsellors - Higher Education Participation and Partnership Program - 1 FTE Student Wellbeing Counsellor - Student Services and Amenities Fee (SSAF) - 2 FTE Reception - Higher Education Participation and Partnership Program. 
- Combined with student health overall funding for student health comes from student levy, PHO capitation and patient paying for both counselling and health.

- $50 \%$ funded by the university $50 \%$ self funded (revenue earned from private practitioners).

- Community Service Obligation Funding under Smart and Skilled VET Funding model.

- We are funded entirely by SSAF. My budget is given to me. I'm not consulted with but must maintain the budget for all costs associated with service provision.

- Income generation.

- Some international students' insurance bulk billing.

- I am unsure of funding input.

Q20 Additional budget allocation as a consequence of increases in overall enrolment

More than eighty-nine percent $(89.47 \%$ ) of managers reported that the counselling service had not received additional funding in line with increased enrolment.

Over ten percent $(10.53 \%)$ of managers reported that the counselling service had received additional funding in line with increased enrolment.

Comments about additional funding included two comments indicating the size of funding increased by less than one percent being 0.14 and 0.2 percent respectively.

Another indicated the funding increase was due to a new campus being opened.

Q21 Given additional funds provided on the basis of increased enrolment numbers, is funding now rolling into 2018

All services $(\mathrm{N}=4)$ that received additional funding due to enrolment increases indicated that the additional funding was now recurrent.

\section{Q22 Line Management Direct Reports}

The spread of direct reports to the counselling service manager encompassed none to more than 30 . The variation between institutions was considerable. There were twenty-two (22) variations in the number of direct reports across thirty (30) responding managers. Three (3) managers reported direct line management for nine (9) staff and another three managers reported direct line management for seventeen (17) staff.

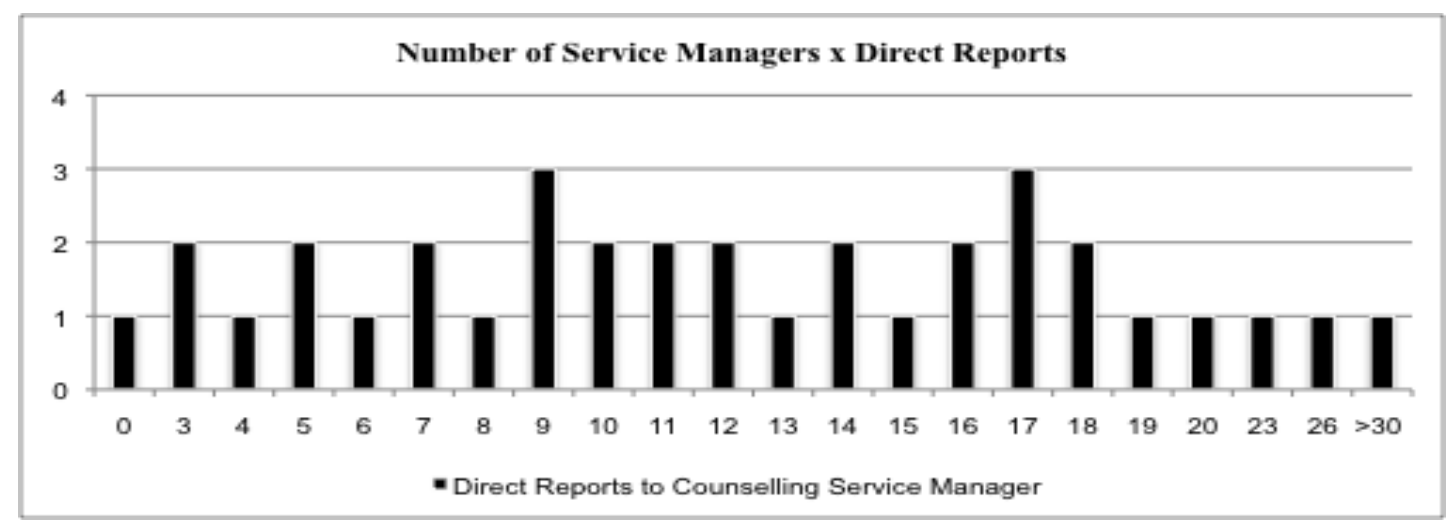

Figure 6

Q23 Professionals employed within the Counselling Service

The most frequent responses received were psychologist and psychologist with endorsed specialty 
area, social workers and counsellors.

Table 4

\begin{tabular}{|l|c|c|}
\hline Professionals employed within Counselling Services & $\begin{array}{c}\text { Percentage of } \\
\text { Responses }\end{array}$ & $\begin{array}{c}\text { Number of } \\
\text { Responses }\end{array}$ \\
\hline Psychologist/s (generalist) & $77.14 \%$ & 27 \\
\hline $\begin{array}{l}\text { Psychologists with endorsed specialty areas (via National } \\
\text { Registration) }\end{array}$ & $62.86 \%$ & 22 \\
\hline Social worker/s & $62.86 \%$ & 22 \\
\hline Psychiatrist/s & $8.57 \%$ & 3 \\
\hline Mental health worker/s & $8.57 \%$ & 3 \\
\hline $\begin{array}{l}\text { Counsellor (background other than psychologist or social } \\
\text { worker) }\end{array}$ & $37.14 \%$ & 13 \\
\hline Mental health educator/s & $5.71 \%$ & 2 \\
\hline Psychiatric nurse/s & $5.71 \%$ & 2 \\
\hline Nurse/s with experience in health, mental health treatment & $8.57 \%$ & 3 \\
\hline General Practitioners with mental health expertise & $2.86 \%$ & 1 \\
\hline Other (please specify) & $25.71 \%$ & 9 \\
\hline
\end{tabular}

Other category responses specified: Occupational Therapist; Psychotherapists; Educators and Learning Designers; Doctors and nurses located in a Health Service working alongside Counselling Service staff.

Q24 Number of direct reports to manager role, not engaged in counselling, who undertake administrative or project work within the counselling service (across all campuses).

Thirteen (13) managers (37.14\%) reported having 2 staff providing administrative or project work. Eight (8) managers (22.86\%) reported having zero (0) staff engaged in administrative or project work.

Seven (7) managers (20\%) reported having one (1) staff member engaged in administrative or project work.

Three (3) managers (8.57\%) reported having three (3) staff members engaged in administrative or project work.

Two (2) managers (5.71\%) reported having five (5) staff members employed in administrative or project work.

One (1) manager (2.86\%) reported having seven (7) staff members employed in administrative or project work.

Table 5

\begin{tabular}{|c|c|c|}
\hline $\begin{array}{c}\text { Number employed in non- } \\
\text { counselling roles }\end{array}$ & $\begin{array}{c}\text { Percentage of } \\
\text { managers }\end{array}$ & $\begin{array}{c}\text { Number of } \\
\text { managers }\end{array}$ \\
\hline 0 & $22.86 \%$ & 8 \\
\hline 1 & $20.00 \%$ & 7 \\
\hline 2 & $37.14 \%$ & 13 \\
\hline 3 & $8.57 \%$ & 3 \\
\hline 4 & $2.86 \%$ & 2 \\
\hline 5 & $5.71 \%$ & 1 \\
\hline 7 & $2.86 \%$ & \\
\hline
\end{tabular}

Other Specified:

- All are admin staff who provide a service to all professionals within the combined Centre.

- Wellness co-ordinator.

- 2 part-time reception staff. 
Q25 Established full time effective (FTE) professional staffing load for counselling service provision across all campuses

Thirty-five (35) different responses for this question were reported. There is considerable variation in the counsellor FTE provided across institutions. The effectiveness of the counsellor FTE in the delivery of individual counselling services arguably has a relationship to the institution's enrolment numbers. So to enhance understanding of the benchmarking a comparison of the data provided for institution's enrolment numbers alongside the FTE for counselling staff as reported is provided in Table 6. Missing data is identified as 'not available' (N/A).

Table 6

\begin{tabular}{|c|c|c|c|c|}
\hline $\begin{array}{l}\text { FTE - excluding } \\
\text { manager role }\end{array}$ & $\begin{array}{l}\text { Frequency of } \\
\text { Response }\end{array}$ & $\begin{array}{c}\text { Institution \# } 1 \\
\text { Number } \\
\text { enrolled March } \\
2017 \\
\end{array}$ & $\begin{array}{c}\text { Institution \# } 2 \\
\text { Number } \\
\text { enrolled March } \\
2017 \\
\end{array}$ & $\begin{array}{c}\text { Institution \# 3 } \\
\text { Number } \\
\text { enrolled March } \\
2017 \\
\end{array}$ \\
\hline & 1 & $300-400$ & & \\
\hline 0.9 & 1 & 3,000 & & \\
\hline 1 & 1 & 9,507 & & \\
\hline 1.4 & 1 & $\mathrm{~N} / \mathrm{A}$ & & \\
\hline 2 & 1 & 6,000 & & \\
\hline 2.9 & 1 & 23,847 & & \\
\hline 3 & 2 & 18,887 & 26,547 & \\
\hline 3.4 & 1 & 25,841 & & \\
\hline 5 & 3 & 17,853 & 21,371 & 37,788 \\
\hline 5.1 & 1 & 20,898 & & \\
\hline 5.2 & 1 & N/A- & & \\
\hline 6.8 & 1 & N/A & & \\
\hline 7 & 1 & 42,208 & & \\
\hline 7.4 & 2 & 23,109 & 30,767 & \\
\hline 7.9 & 2 & 26,975 & 24,891 & \\
\hline 8 & 1 & 28,000 & & \\
\hline 8.8 & 1 & 52,331 & & \\
\hline 10 & 3 & 23,000 & N/A & N/A \\
\hline 10.82 & 1 & 51,338 & & \\
\hline 11.2 & 1 & 36,746 & & \\
\hline 11.6 & 1 & $\mathrm{~N} / \mathrm{A}$ & & \\
\hline 11.8 & 1 & 70,000 & & \\
\hline 12 & 1 & 67,000 & & \\
\hline 12.4 & 1 & 58,210 & & \\
\hline 12.6 & 1 & 66,928 & & \\
\hline 13 & 1 & 38,000 & & \\
\hline 13.4 & 1 & 40,209 & & \\
\hline 18 & 1 & 73,807 & & \\
\hline
\end{tabular}

Excluding the service manager eighteen (18) was the highest FTE for professional staff providing counselling services servicing 73,807 enrolled students. At the other end of the range no additional FTE was provided beyond the counsellor managing the service at one institution where the enrolment was less than 500 students.

Between the bookends of the range for FTE (counsellors) 0 to18 there is considerable variation. Some of that variation could be accounted for by the institution's enrolment load. Table 7 highlights the FTE variation for different enrolment loads reported by managers across the sector. Three (3) institutions satisfied the International AUCCCD survey benchmark of 1 counsellor to < 3,000 students. Twenty-three (23) institutions satisfied the ANZSSA recommended benchmark of 1 
counsellor to 6,000 students when considering additional student support services readily available. More needs to be understood about the factors influencing this variation in resourcing the FTE for counsellor staffing across the sector.

Table 7

\begin{tabular}{|c|c|c|c|}
\hline $\begin{array}{c}\text { Enrolment } \\
\text { load low to } \\
\text { higher }\end{array}$ & $\begin{array}{l}\text { Counsellor } \\
\text { FTE in } \\
\text { addition to } \\
\text { the } \\
\text { manager } \\
\text { role }\end{array}$ & $\begin{array}{c}\text { Satisfies the recommended } \\
\text { International AUCCCD } \\
\text { benchmark for FTE at } 1 \\
\text { counsellor to }<3,000 \\
\text { enrolled students }\end{array}$ & $\begin{array}{l}\text { Satisfies the recommended } \\
\text { ANZSSA benchmark minimum } \\
\text { FTE of } 1 \text { counsellor to } 6,000 \\
\text { enrolled students if multiple } \\
\text { other student support services } \\
\text { provided }\end{array}$ \\
\hline $300-400$ & 0 & $x$ & $\checkmark$ \\
\hline 3,000 & 0.9 & $x$ & $\checkmark$ \\
\hline 6,000 & 2 & $x$ & $\checkmark$ \\
\hline 9,507 & 1 & $x$ & $x$ \\
\hline 17,853 & 5 & $x$ & $\checkmark$ \\
\hline 18,887 & 3 & $x$ & $x$ \\
\hline 20,898 & 5.1 & $x$ & $\checkmark$ \\
\hline 21,371 & 5 & $x$ & $\checkmark$ \\
\hline 23,000 & 10 & $\checkmark$ & $\checkmark$ \\
\hline 23,109 & 7.4 & $x$ & $\checkmark$ \\
\hline 23,847 & 2.9 & $x$ & $x$ \\
\hline 24,891 & 7.9 & $x$ & $\checkmark$ \\
\hline 25,841 & 3.4 & $x$ & $x$ \\
\hline 26,547 & 3 & $x$ & $x$ \\
\hline 26,975 & 7.9 & $x$ & $\checkmark$ \\
\hline 28,000 & 8 & $x$ & $\checkmark$ \\
\hline 30,767 & 7.4 & $x$ & $\checkmark$ \\
\hline 36,746 & 11.2 & $x$ & $\checkmark$ \\
\hline 37,788 & 5 & $x$ & $x$ \\
\hline 38,000 & 13 & $\checkmark$ & $\checkmark$ \\
\hline 40,209 & 13.4 & $\checkmark$ & $\checkmark$ \\
\hline 42,208 & 7 & $x$ & $\checkmark$ \\
\hline 51,338 & 10.82 & $x$ & $\checkmark$ \\
\hline 52,331 & 8.8 & $x$ & $\checkmark$ \\
\hline 58,210 & 12.4 & $x$ & $\checkmark$ \\
\hline 66,928 & 12.6 & $x$ & $\checkmark$ \\
\hline 67,000 & 12 & $x$ & $\checkmark$ \\
\hline 70,000 & 11.8 & $x$ & $\checkmark$ \\
\hline 73,807 & 18 & $x$ & $\checkmark$ \\
\hline
\end{tabular}

FTE identified for institutions where enrolment data was not available

\begin{tabular}{|l|c|c|c|}
\hline N/A & 1.4 & \\
\cline { 1 - 1 } N/A & 5.2 & \multirow{3}{*}{ Not able to be determined } & \multirow{3}{*}{ Not able to be determined } \\
\cline { 1 - 2 } N/A & 6.8 & \\
\hline N/A & 10 & \\
\hline N/A & 10 & \\
\hline N/A & 11.6 &
\end{tabular}

See also reporting on the counsellor to student ratio in Q26-29.

Three (3) managers reported an FTE of 0 to 1 . Enrolments reported for 2 (two) were $<3,000$ and the other was $<10,000$. 
Two (2) managers reported counsellor FTE between 1 and 2. Enrolment load reported was N/A and $>9,500$.

Three (3) managers reported counsellor FTE between 2 and 3. Enrolment loads were 18,887, 23,847 and 26,547 .

One (1) manager reported a counsellor FTE between 3 and 3.5. The enrolment load reported was 25,841 .

Five (5) managers reported a counsellor FTE between 5 and 5.2. Enrolment loads were reported as N/A, 17,853, 20,898, 21,371 and 37,788.

One (1) manager reported a counsellor FTE of 6.8. Enrolment load data was N/A.

Five (5) managers reported a counsellor FTE between 7 and 7.9. Enrolment loads were reported as $23,109,24,891,26,975,30,767$ and 42,208.

Two (2) managers reported counsellor FTE as 8 to 8.8 . Enrolment loads reported were 28,000 and 52,331 .

Two (2) managers reported counsellor FTE as 10 to 10.82. Enrolment loads reported were 23,000 and 51,338 .

Three (3) managers reported counsellor FTE as 11.2 to 11.8. Enrolment loads reported were N/A, 36,746 and 70,000 .

Three (3) managers reported counsellor FTE as 12 to 12.6. Enrolment loads reported were 58,210, 66,928 and 67,000 .

Two (2) managers reported counsellor FTE as 13 to 13.4. Enrolment loads reported were 38,000 and 40,209.

One (1) manager reported the counsellor FTE as 18 . The enrolment load was reported as 73,807.

The range for number of students enrolled at March 2017 is 400 to 73,800.

Six (6) managers reported enrolment data as N/A.

Four (4) managers reported enrolments of $<10,000$.

Eight (8) managers reported enrolments between 15,000 and 25,000 students.

Five (5) managers reported enrolments between $>25,000$ to 35,000 .

Five (5) managers reported enrolments between $>35,000$ to 45,000 .

Two (2) managers reported enrolments between $>45,000$ to 55,000 .

Five (5) managers reported enrolments of $>55,000$.

Q26 Established administrative and project staffing FTE load is there within your counselling service (inclusive of all campuses)

The most commonly reported ( $\mathrm{N}=10)$ staffing for administrative and project staff was 1 FTE.

Six (6) respondents reported 0 FTE for administrative and project work.

Four (4) respondents reported 2 FTE and two (2) respondents reported 3 FTE.

Other respondents reported FTE across a broad range (0.2 FTE - 8 FTE). 


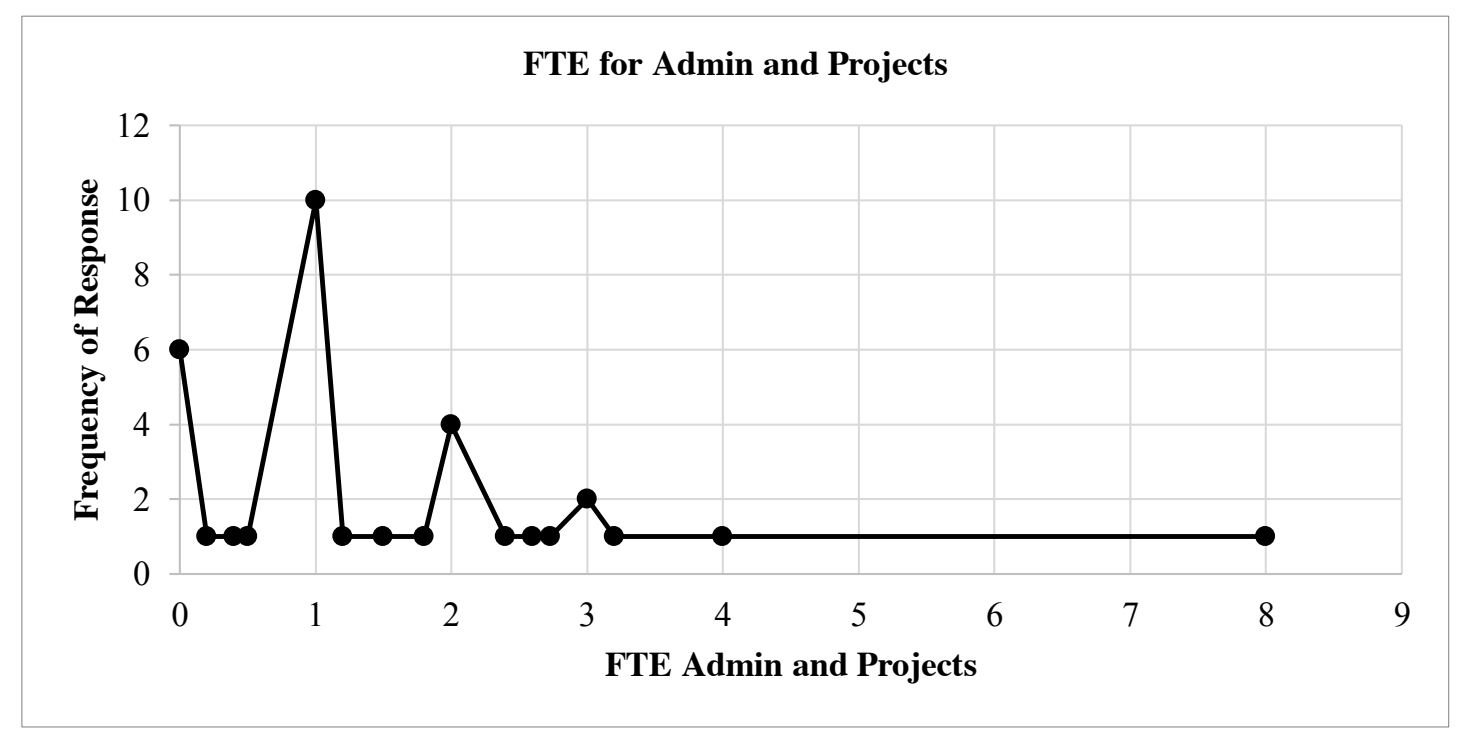

Figure 7

Q27 Professional staff to student ratio provided by your service for the calendar year of 2016

Seven (7) respondents reported a professional staff to student ratio of 1 to less than 3,000.

Ten (10) respondents reported a professional staff to student ratio of 1to 3,001- 4,000.

Six (6) respondents reported a professional staff to student ratio of 1 to 4,001-5,000.

Six (6) respondents reported a professional staff to student ratio of 1 to 5,001-6,000.

One (1) respondent reported a professional staff to student ratio of 1 to 6,001-7,000.

Three (3) respondents reported a professional staff to student ratio of 1 to 7,001-8,000.

Two (2) respondents reported a professional staff to student ratio of 1 to $11,000-12,000$.

Three (3) respondents did not provide a professional staff to student ratio. 
2016 Counsellor to Student Ratio x Frequency of Response

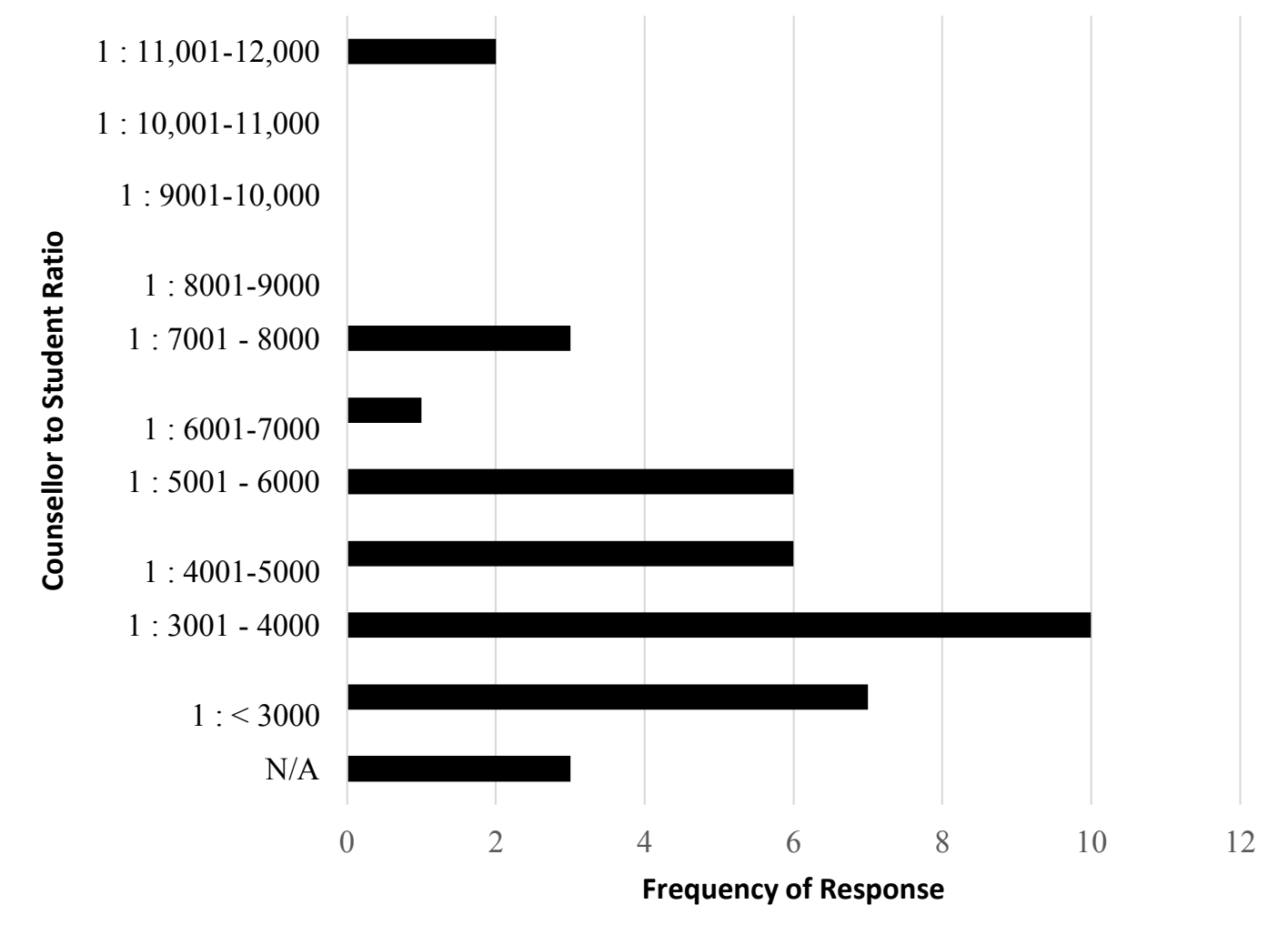

Figure 8

Q28 Professional staff to student ratio provided by your service for the calendar year of 2017

Ten (10) respondents reported a professional staff to student ratio of 1 to less than 3000.

Nine (9) respondents reported a professional staff to student ratio of 1 to 3001- 4000.

Six (6) respondents reported a professional staff to student ratio of 1 to 5001- 6000.

Four (4) respondents reported a professional staff to student ratio of 1 to $4001-5000$.

Three (3) respondents reported a professional staff to student ratio of 1 to $7001-8000$.

One (1) respondent reported a professional staff to student ratio of 1 to $6001-7000$.

One (1) respondent reported a professional staff to student ratio of 1 to $8001-9000$.

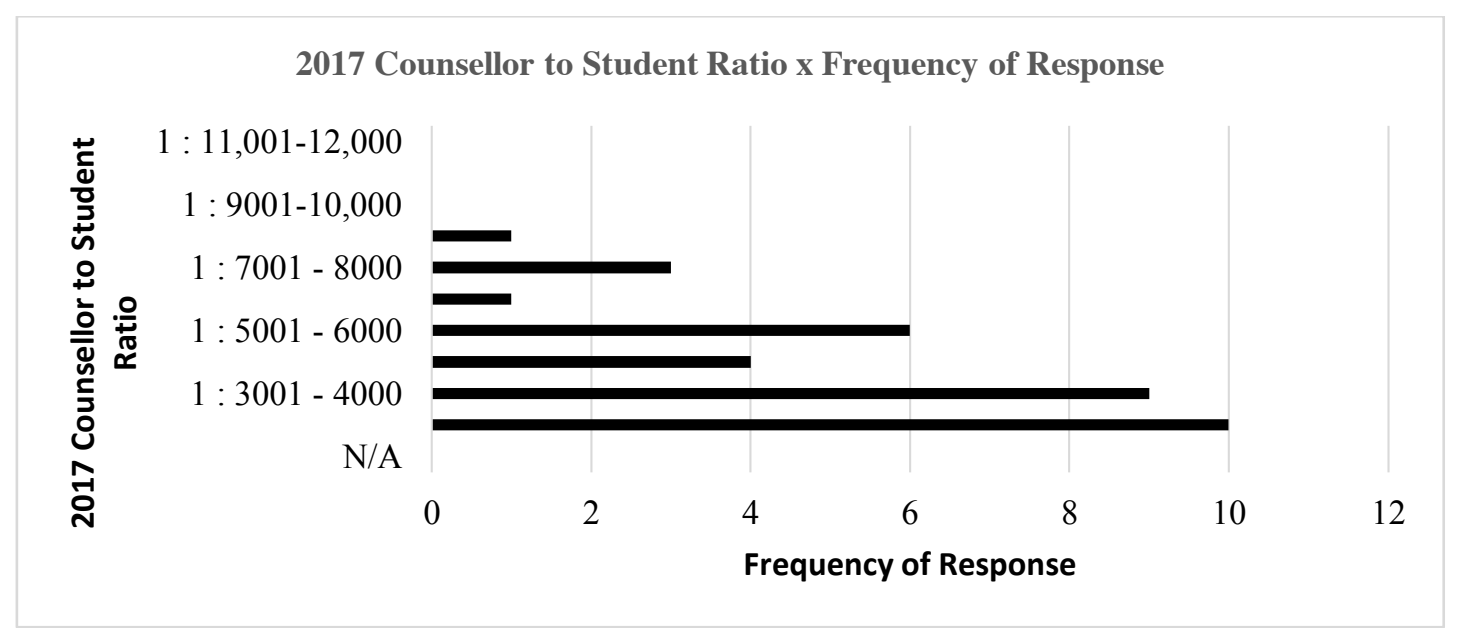

Figure 9 
Q29 Was the staffing to student ratio provided in 2017 considered adequate for the expected/core service delivery

Twenty-five (25) of responding managers $(71.43 \%)$ indicated that the staffing to student ratio provided for the counselling service during 2017 was not adequate for expected core service delivery.

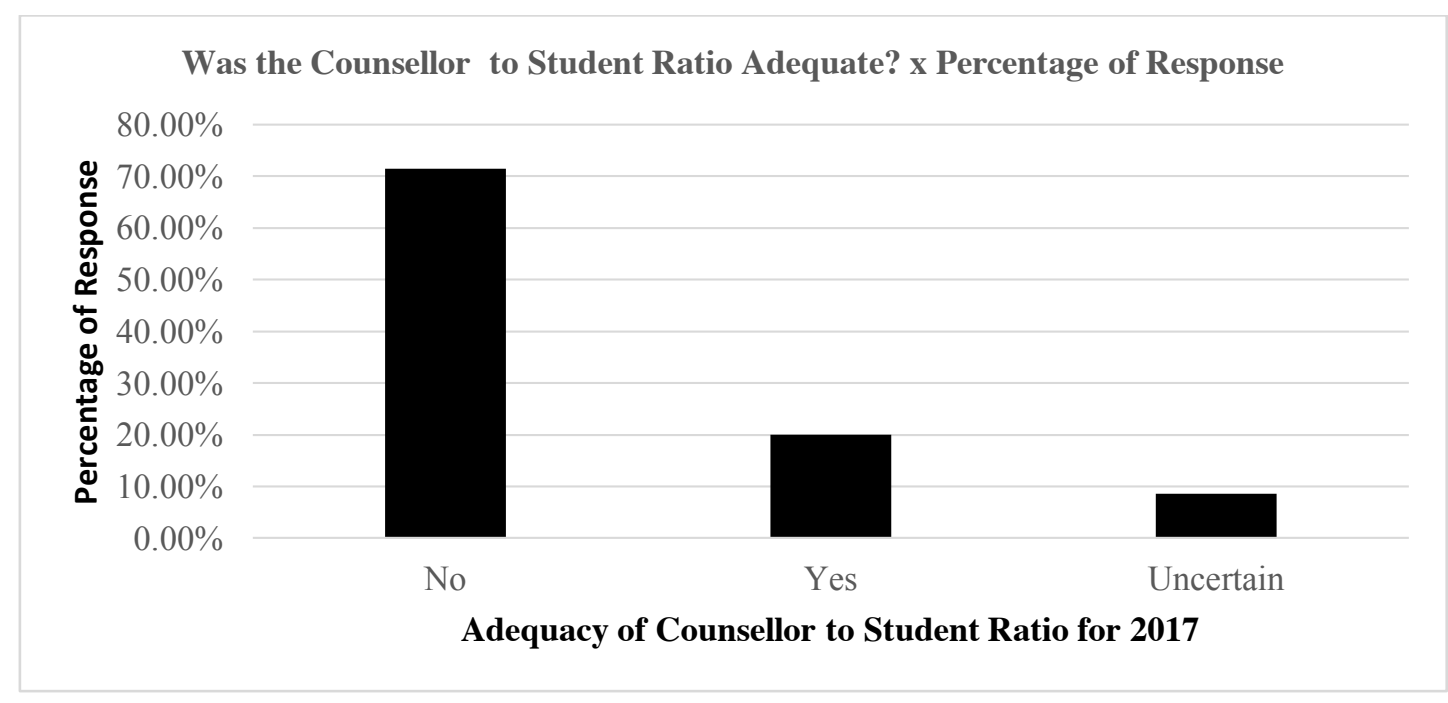

Figure 10

Managers were asked to comment on what they thought would be a reasonable staff to student ratio to address service delivery adequately at their institution. The majority who provided a ratio identified a ratio between 1500-2500 students per staffing FTE.

Table 8

\begin{tabular}{|c|c|c|}
\hline Collapsed groupings & Suggested ratio & Frequency of response \\
\hline \multirow{3}{*}{1 counsellor to: } & $1500-2500$ & 11 \\
\cline { 2 - 3 } & $3000-3500$ & 4 \\
\cline { 2 - 3 } & $4000-5000$ & 2 \\
\hline & $\begin{array}{c}\text { Don't know/Uncertain/ } \\
\text { Comment but no figure given }\end{array}$ & 4 \\
\hline
\end{tabular}

Q30 International benchmarking information from International Association of Counselling Standards (IACS., n.d.) in the Standards for University and College Counselling Services (IACS., 2010 , amended $2013 \& 2016)$

The 2015-2016 AUCCCD Survey (n.d) reports the professional staff to student ratio across the academic year in universities (both public and private with enrolments ranging from 15,001 to $>35,001)$ as:

15,001-20,000 = average of 2,139 students to 1 x paid professional staff member 20,001-25,000 = average 2,402 students to $1 x$ paid professional staff member 25,001-30,000 = average 2,567 students to $1 \times$ paid professional staff member 30,001-35,000 = average 2,471 students to $1 \times$ paid professional staff member $>35,001$ = average 2,624 students to 1 xaid professional staff member.

The International Association of Counselling Standards (IACS) (n.d.) in the Standards for University and College Counselling Services states on page 13:

V.C.1. Every effort should be made to maintain minimum staffing ratios $n$ the range of one FTE professional staff member (excluding trainees) for every 1,000-1500 students, depending on services offered and other campus mental health agencies.

Managers were then asked to choose the professional staff to student ratio they considered adequate 
for the Australian/New Zealand tertiary education context.

Twenty-one (21) managers $(61.76 \%)$ indicated that the staff to student ratio considered ideal for the Australian/New Zealand Higher Education context lay between the values of 1 to 1,500 - 2,500.

One other response:

Unsure - perhaps 1:2000??

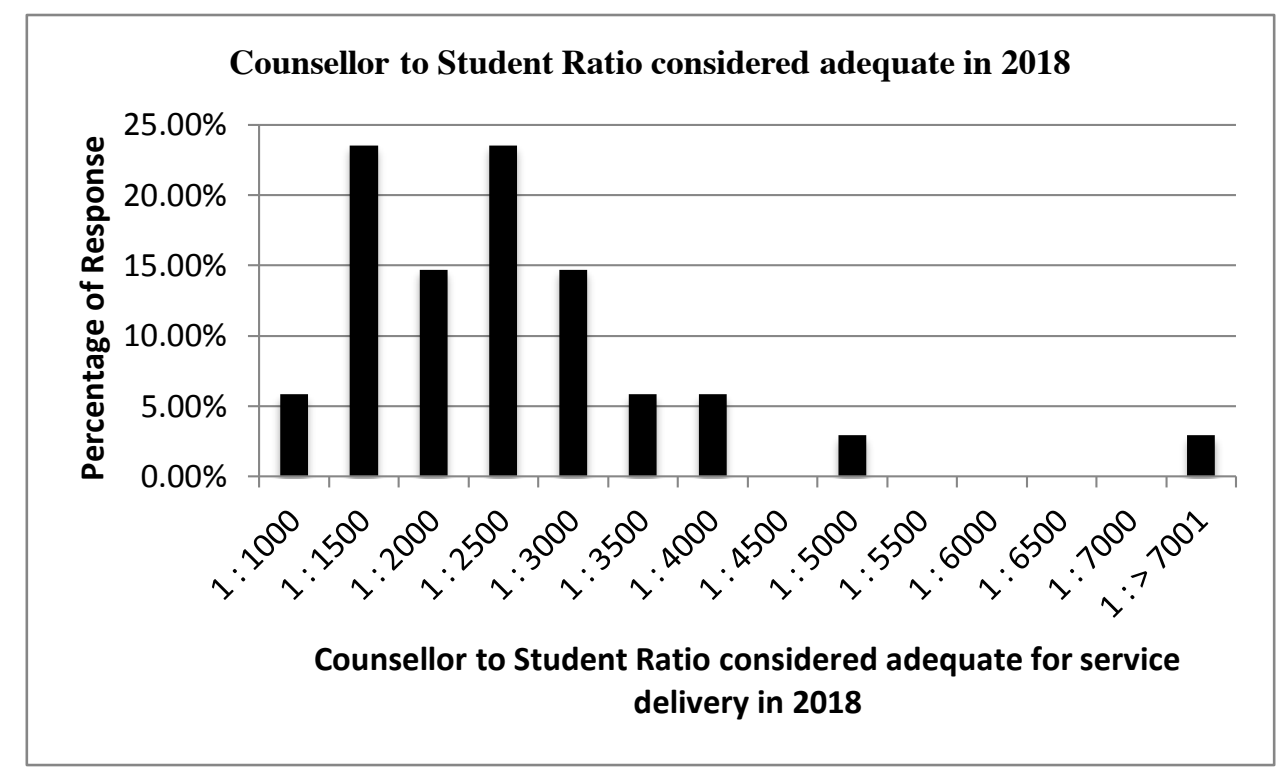

Figure 11

Twenty-nine (29) managers' responses $(82.6 \%)$ indicated that an adequate staff to student ratio would be equal to or less than 1 to 3,000 .

Collapsed categories: Adequate staff to student ratio x Number of Responses in 2018

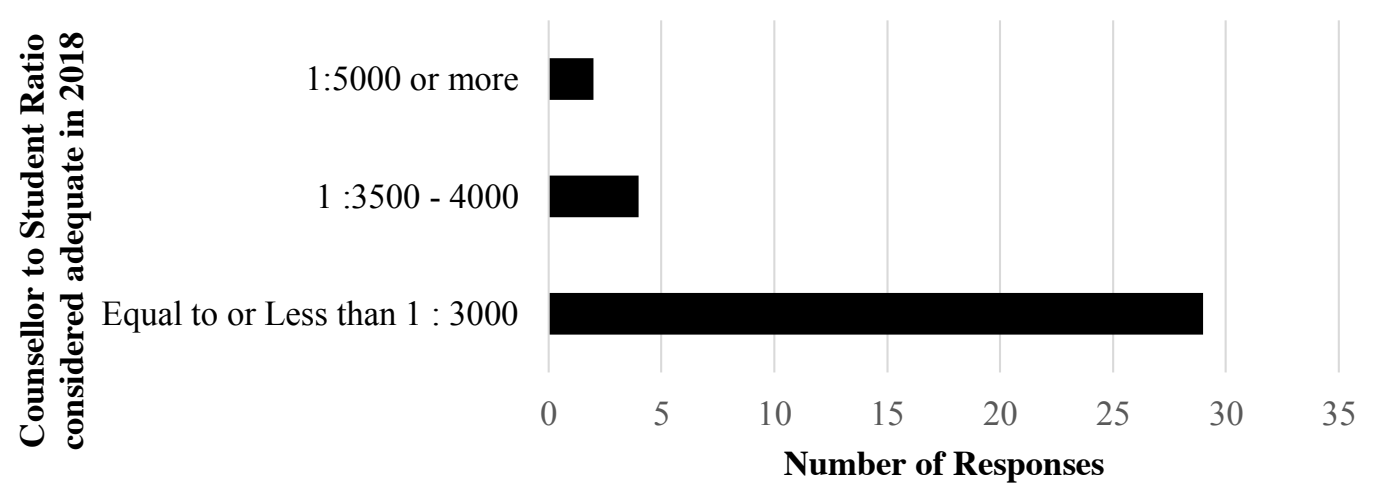

Figure 12

Q31 Professional staff to student ratio true of the counselling service in manager's institutional context

The current ratio range for professional staff per student across institutions reported by respondents is $1: 1000$ to 1 to $>7000$.

Approximately $15 \%(15.15 \%)$ of respondents reported equally for three different ratios being: 1 to 3500 ; 1 to 5500 ; and 1 to $>7000$. 
Table 9

\begin{tabular}{|c|c|}
\hline $\begin{array}{c}\text { Current Professional staff to Student } \\
\text { Ratio }\end{array}$ & Number of Institutions \\
\hline 1 to 1000 & 1 \\
\hline 1 to 1500 & 0 \\
\hline 1 to 2000 & 1 \\
\hline 1 to 2500 & 4 \\
\hline 1 to 3000 & 4 \\
\hline 1 to 3500 & 5 \\
\hline 1 to 4000 & 3 \\
\hline 1 to 4500 & 2 \\
\hline 1 to 5000 & 2 \\
\hline 1 to 5500 & 5 \\
\hline 1 to 6000 & 1 \\
\hline 1 to 6500 & 0 \\
\hline 1 to 7000 & 0 \\
\hline 1 to $>7001$ & 5 \\
\hline
\end{tabular}

Q32 Types of professionals that managers would like to add (wish list) or increase FTE representation within their service team

There were six (6) professional roles most desired as team additions by the respondents: psychologists (generalists); psychologist (with endorsed specialty area); social workers; psychiatrists; mental health educators; mental health workers.

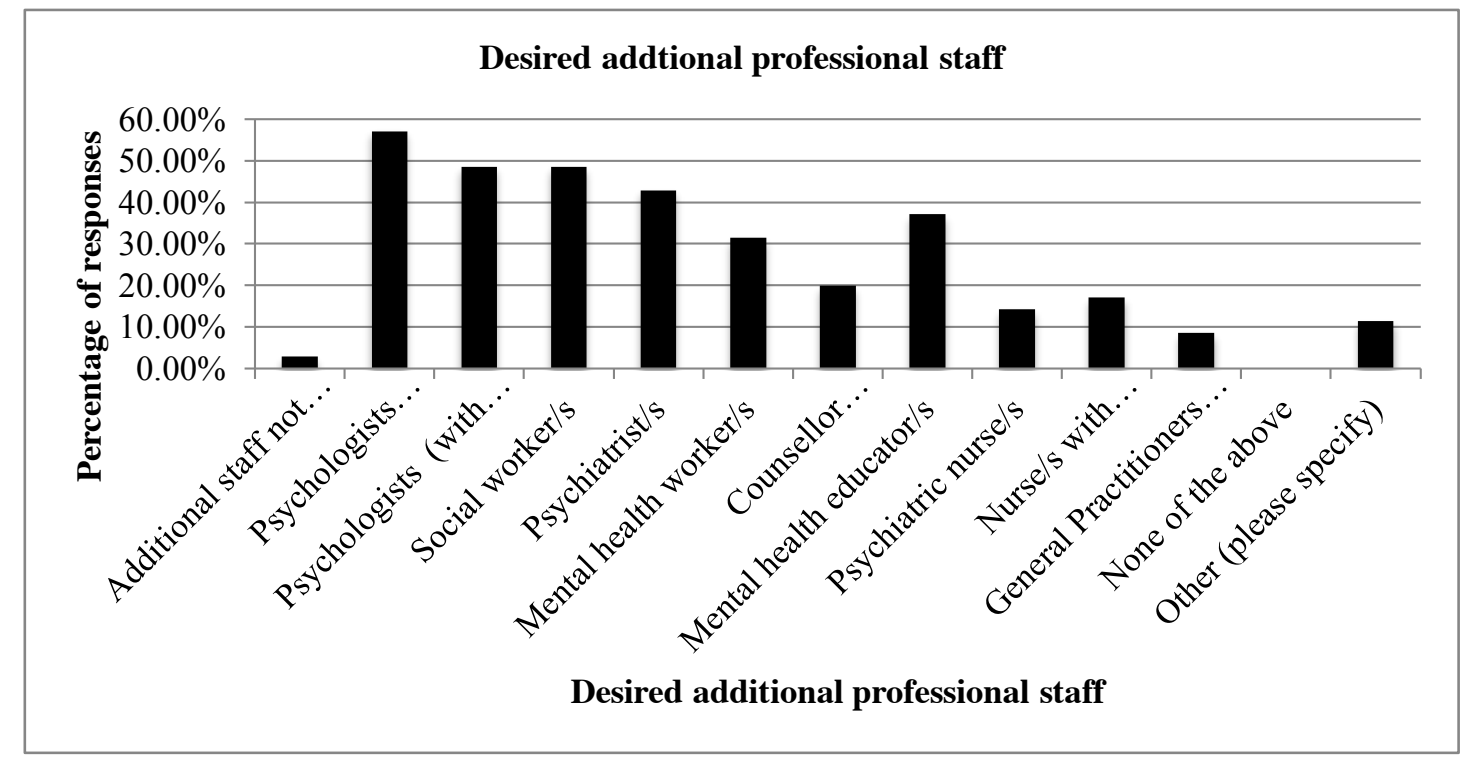

Figure 13

Over half of the managers (57.14\%) responded that they would like to add psychologists (generalists) to their team.

Forty-eight percent $(48.57 \%)$ of managers indicated they wanted to add psychologists (endorsed speciality) and social workers to their team.

Slightly more than forty-two percent $(42.86 \%)$ of managers indicated that they wanted psychiatrists and approximately thirty-seven percent $(37.14 \%)$ wanted mental health educators.

Mental health workers were desired by slightly more than thirty-one percent (31.43\%) of managers. 
Q33 Percentage of time utilised by service delivery areas:

Some service delivery areas were common to all respondents $(\mathrm{N}=33)$ inclusive of:

- Individual counselling appointments.

- Urgent/emergency appointments.

- Consultation with university staff about specific students of concern.

- Mental health awareness lectures/workshops (to staff or students) including Mental Health First Aid were provided by almost all counselling services $(\mathrm{N}=30)$.

- Twenty-nine (29) managers indicated that services included psycho-educational workshops;

- Twenty-six (26) managers offered workshops or presentations in scheduled class time; and Twenty-three (23) indicated that counselling service activities included projects or programs designed to target ALL enrolled students;

- Fewer that twenty $(<20)$ managers reported offering the following: Projects or programs designed to target prospective students (17); and other activities not linked to categories listed above (16).

Managers were not asked to identify the other activities.

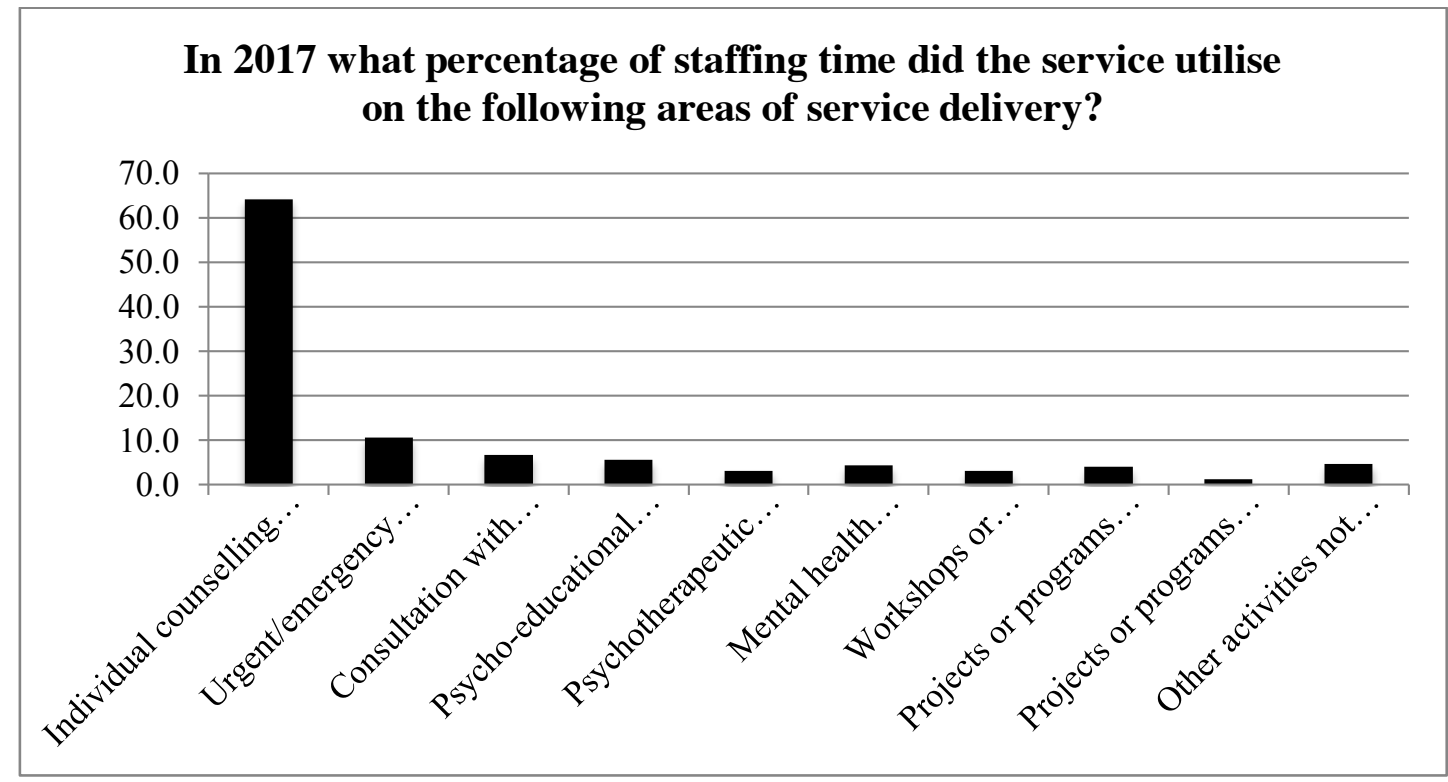

Figure 14

\section{a) Individual counselling}

Institutional responses aggregated into four groupings (percentage of time utilised on individual counselling) highlight that the majority $(72.72 \%)$ utilise between sixty $(60 \%)$ and seventy-nine (79\%) percent of service delivery time on individual counselling. Only two (2) institutions (6\%) allocated more service delivery time to individual counselling and just seven (7) (21.21\%) offered less.

Table 10
\begin{tabular}{|c|c|}
\hline \multicolumn{2}{|c|}{ Individual counselling } \\
\hline Number of Responses & Percentage of time \\
\hline 2 & $80-89$ \\
\hline 11 & $70-79$ \\
\hline 13 & $60-69$ \\
\hline 7 & $50-59$ \\
\hline
\end{tabular}




\section{b) Percentage of staff time utilised by Urgent/Emergency appointments}

The range of responses was two (2) percent to twenty-five (25) percent. The most frequent response was ten (10) percent of staff time provided by twelve (12) managers.

Table 11

\begin{tabular}{|c|c|}
\hline \multicolumn{2}{|c|}{ Urgent/Emergency Appointments } \\
\hline Number of Responses & Percentage of time \\
\hline 2 & 2 \\
\hline 1 & 3 \\
\hline 1 & 4 \\
\hline 2 & 5 \\
\hline 1 & 6 \\
\hline 12 & 7 \\
\hline 5 & 10 \\
\hline 1 & 15 \\
\hline 2 & 18 \\
\hline 1 & 20 \\
\hline 1 & 23 \\
\hline
\end{tabular}

\section{c) Percentage of staff time utilised by consultations with staff about students of concern}

The range of responses was one (1) percent to eighteen (18) percent. The most frequent response was five (5) percent of staff time provided by thirteen (13) managers. The second frequent response was ten (10) percent of staff time provided by nine (9) managers.

\section{Table 12}

\begin{tabular}{|c|c|}
\hline \multicolumn{2}{|c|}{ Consultation with university staff about specific students of concern } \\
\hline Percentage of staff time & Number of Responses \\
\hline 1 & 2 \\
\hline 3 & 5 \\
\hline 4 & 1 \\
\hline 5 & 13 \\
\hline 10 & 9 \\
\hline 11 & 1 \\
\hline 15 & 1 \\
\hline 18 & 1 \\
\hline
\end{tabular}

\section{d) Percentage of staff time utilised by psychoeducational workshops}

The range reported was zero (0) to fifteen (15) percent of staff time. The most frequent response was five (5) percent of staff time provided by seventeen (17) managers.

Table 13

\begin{tabular}{|c|c|}
\hline \multicolumn{2}{|c|}{ Psycho-educational workshops } \\
\hline Percentage of time & Number of Responses \\
\hline 0 & 1 \\
\hline 1 & 1 \\
\hline 2 & 1 \\
\hline 3 & 2 \\
\hline 4 & 1 \\
\hline 5 & 17 \\
\hline 6 & 0 \\
\hline 7 & 0 \\
\hline 8 & 1 \\
\hline
\end{tabular}




\begin{tabular}{|c|c|}
\hline \multicolumn{2}{|c|}{ Psycho-educational workshops } \\
\hline Percentage of time & Number of Responses \\
\hline 9 & 0 \\
\hline 10 & 4 \\
\hline 11 & 0 \\
\hline 12 & 0 \\
\hline 13 & 0 \\
\hline 14 & 0 \\
\hline 15 & 1 \\
\hline
\end{tabular}

\section{e) Percentage of staff time utilised by psychotherapeutic workshops}

The range reported was zero (0) to fifteen (15) percent of staff time.

The most frequent response was zero (0) percent of staff time provided by eight (8) managers.

Table 14

\begin{tabular}{|c|c|}
\hline \multicolumn{2}{|c|}{ Psychotherapeutic workshops } \\
\hline Percentage of time & Number of Responses \\
\hline 0 & 8 \\
\hline 1 & 0 \\
\hline 2 & 3 \\
\hline 3 & 2 \\
\hline 4 & 0 \\
\hline 5 & 6 \\
\hline 6 & 0 \\
\hline 7 & 1 \\
\hline 8 & 0 \\
\hline 9 & 0 \\
\hline 10 & 0 \\
\hline 11 & 0 \\
\hline 12 & 0 \\
\hline 13 & 0 \\
\hline 14 & 0 \\
\hline 15 & 1 \\
\hline
\end{tabular}

f) Percentage of staff time utilised by mental health awareness

The range reported was zero $(0)$ to ten $(10)$ percent of staff time.

The most frequent response was five (5) percent of staff time provided by nine (9) managers.

Table 15

\begin{tabular}{|c|c|}
\hline \multicolumn{2}{|c|}{ Mental Health Awareness } \\
\hline Percentage of Time & Number of Responses \\
\hline 0 & 2 \\
\hline 1 & 4 \\
\hline 2 & 5 \\
\hline 3 & 3 \\
\hline 4 & 1 \\
\hline 5 & 9 \\
\hline 6 & 0 \\
\hline 7 & 0 \\
\hline 8 & 1 \\
\hline 9 & 0 \\
\hline 10 & 5 \\
\hline
\end{tabular}

Journal of the Australian and New Zealand Student Services Association: 
g) Percentage of staff time utilised by workshops or presentations scheduled within class time

The range reported was zero $(0)$ to ten (10) percent of staff time.

The most frequent response was five (5) percent of staff time provided by nine (9) managers.

Table 16

\begin{tabular}{|c|c|}
\hline \multicolumn{2}{|c|}{ Workshops or presentations in scheduled class time } \\
\hline Percentage of time & Number of Responses \\
\hline 0 & 3 \\
\hline 1 & 5 \\
\hline 2 & 6 \\
\hline 3 & 1 \\
\hline 4 & 1 \\
\hline 5 & 9 \\
\hline 6 & 0 \\
\hline 7 & 0 \\
\hline 8 & 0 \\
\hline 9 & 0 \\
\hline 10 & 1 \\
\hline
\end{tabular}

h) Percentage of staff time utilised by projects or programs designed to target ALL enrolled students

The range reported was zero $(0)$ to ten $(10)$ percent of staff time.

The most frequent response was five (5) percent of staff time provided by eight (8) managers.

Table 17

\begin{tabular}{|c|c|}
\hline \multicolumn{2}{|c|}{ Projects or programs designed to target ALL enrolled students } \\
\hline Percentage of Staff Time & Number of Responses \\
\hline 0 & 2 \\
\hline 1 & 4 \\
\hline 2 & 4 \\
\hline 3 & 1 \\
\hline 4 & 0 \\
\hline 5 & 8 \\
\hline 6 & 0 \\
\hline 7 & 1 \\
\hline 8 & 0 \\
\hline 9 & 0 \\
\hline 10 & 3 \\
\hline
\end{tabular}

i) Percentage of staff time utilised by projects or programs designed to target prospective students.

The majority of managers reported utilising no time on programs targeting prospective students.

Table 18

\begin{tabular}{|c|c|}
\hline \multicolumn{2}{|c|}{ Projects or programs designed to target prospective students } \\
\hline Percentage of Staff Time & Number of Responses \\
\hline 0 & 9 \\
\hline 1 & 3 \\
\hline 2 & 2 \\
\hline 5 & 3 \\
\hline
\end{tabular}

j) Percentage of staff time utilised by activities other than those identified a) to i) above.

Other activities utilised between zero (0) and twenty-six (26) percent of staff time. The majority of 
services utilised between one (1) and five (5) percent of staff time on other activities.

Table 19

\begin{tabular}{|c|c|}
\hline \multicolumn{2}{|c|}{ Other Activities } \\
\hline Percentage of Staff Time & Number of Responses \\
\hline 0 & 5 \\
\hline 1 & 1 \\
\hline 2 & 2 \\
\hline 3 & 2 \\
\hline 4 & 3 \\
\hline 5 & 1 \\
\hline 20 & 1 \\
\hline 26 & 1 \\
\hline
\end{tabular}

Q34 Are the salaries paid to counselling service staff competitive with other relevant employers?

The majority of managers (27) believed that the salaries offered within their service were competitive with other relevant employers.

Table 20

\begin{tabular}{|c|c|}
\hline \multicolumn{2}{|c|}{ Salaries Competitive } \\
\hline Answer Choices & Number of Institutions \\
\hline Yes & 27 \\
\hline No & 5 \\
\hline Don't know & 3 \\
\hline
\end{tabular}

Q35 Student services centrally funded (full or part) and offered separately

There was a broad range of services additional to the counselling service provided for students by institutions.

Academic skills/information was the additional service most frequently provided by the responding institutions $(\mathrm{N}=34)$.

Other popular services offered by almost all the responding institutions included:

- Disability Services $(\mathrm{N}=32)$

- Services for indigenous students (ATSI or Maori or PI) $(\mathrm{N}=31)$

- A dedicated service for international students $(\mathrm{N}=30)$

- Academic advising services $(\mathrm{N}=30)$

The following services were provided by at least nineteen (19) institutions:

- Scholarships Office ( $\mathrm{N}=29)$

- Peer Mentoring Services coordination and support (N=29)

- Reasonable educational accommodation adjustments for students with disability or learning difficulties $(\mathrm{N}=28)$

- Careers and Employment Service (combined) (N=27)

- Religious Centre/Chaplaincy ( $\mathrm{N}=26)$

- Long stay housing and accommodation services $(\mathrm{N}=24)$

- Transition to university/First Year Experience Coordination/Services (N=24) 
- Student Volunteer Programs Coordination/Services ( $\mathrm{N}=23)$

- English language support services $(\mathrm{N}=22)$

- Equity Services $(\mathrm{N}=22)$

- Budgeting and financial advice for students on low incomes $(\mathrm{N}=20)$

- IT and online learning software support services $(\mathrm{N}=20)$

- Medical/Health Service (N=20)

- No or low interest loans service (education or living costs) ( $\mathrm{N}=19)$

The following services were provided by at least ten (10) institutions:

- Treatment and Assessment Clinic staffed by psychology or social work interns under supervision (part of formal program of studies) $(\mathrm{N}=15)$

- Short stay or urgently needed/emergency housing service $(\mathrm{N}=13)$

- Physiotherapy Service $(\mathrm{N}=13)$

- Retention Programs Coordination/Services (N=13)

- Welfare services $(\mathrm{N}=12)$

- Learning engagement and enhancement services for specific cohorts entering under special access programs (LSES, First in Family etc) $(\mathrm{N}=12)$

- Careers Services $(\mathrm{N}=10)$

The following services were offered by provided by less than nine (9) institutions:

- Case management of students referred to or from specialist external service providers $(\mathrm{N}=8)$

- Psychiatric Services (assessment/medication/treatment/longer term psychotherapy) $(\mathrm{N}=8)$

- Mental Health Educator or support services provided by a psychologist or nurse $(\mathrm{N}=7)$

- Psychological assessment of learning difficulties/disability (offered as separate service) $(\mathrm{N}=7)$

- Employment Services $(\mathrm{N}=5)$

- Dental Service $(\mathrm{N}=5)$

- Psychological assessment of learning difficulties/disability (offered by counselling or disability service) $(\mathrm{N}=5)$

- A dedicated service for international students (includes personal counselling/psychological services) $(\mathrm{N}=2)$

Comments provided $(\mathrm{N}=2)$ :

- Not sure if that (sic my responses to this question) is totally accurate

- Chaplaincy provided but not funded by University. Medical/Health is part of the Counselling Service

Managers were invited to tick all other student services funded centrally that applied. (See Table 21.) 
Table 21

\begin{tabular}{|c|c|}
\hline Student services centrally funded (full or part) & $\begin{array}{l}\text { Number of } \\
\text { Institutions }\end{array}$ \\
\hline Academic skills/information literacy services & 34 \\
\hline $\begin{array}{l}\text { Disability Services - provision of educational adjustments and some } \\
\text { support (excluding welfare or counselling support) }\end{array}$ & 32 \\
\hline Services for indigenous students (ATSI or Maori or PI) & 31 \\
\hline $\begin{array}{l}\text { A dedicated service for international students (does not include personal } \\
\text { counselling/psychological services) }\end{array}$ & 30 \\
\hline Academic advising services & 30 \\
\hline Scholarships Office & 29 \\
\hline Peer Mentoring Services coordination and support & 29 \\
\hline $\begin{array}{l}\text { Reasonable educational accommodation adjustments for students with } \\
\text { disability or learning difficulties }\end{array}$ & 28 \\
\hline Careers and Employment Service (combined) & 27 \\
\hline Religious Centre/Chaplaincy & 26 \\
\hline Long stay housing and accommodation services & 24 \\
\hline Transition to university/First Year Experience Coordination/Services & 24 \\
\hline Student Volunteer Programs Coordination/Services & 23 \\
\hline English language support services & 22 \\
\hline Equity Services & 22 \\
\hline Budgeting and financial advice for students on low incomes & 20 \\
\hline IT and online learning software support services & 20 \\
\hline Medical/Health Service & 20 \\
\hline No or low interest loans service (education or living costs) & 19 \\
\hline $\begin{array}{l}\text { Treatment and Assessment Clinic staffed by psychology or social work } \\
\text { interns under supervision (part of formal program of studies) }\end{array}$ & 15 \\
\hline Short stay or urgently needed/emergency housing service & 13 \\
\hline Physiotherapy Service & 13 \\
\hline Retention Programs Coordination/Services & 13 \\
\hline Welfare services & 12 \\
\hline $\begin{array}{l}\text { Learning engagement and enhancement services for specific cohorts } \\
\text { entering under special access programs (LSES, First in Family etc) }\end{array}$ & 12 \\
\hline Careers Services & 10 \\
\hline $\begin{array}{l}\text { Case management of students referred to or from specialist external } \\
\text { service providers }\end{array}$ & 8 \\
\hline $\begin{array}{l}\text { Psychiatric Services (assessment/medication/treatment/longer term } \\
\text { psychotherapy) }\end{array}$ & 8 \\
\hline $\begin{array}{l}\text { Mental Health Educator or support services provided by a psychologist or } \\
\text { nurse }\end{array}$ & 7 \\
\hline $\begin{array}{l}\text { Psychological assessment of learning difficulties/disability (offered as } \\
\text { separate service) }\end{array}$ & 7 \\
\hline Employment Services & 5 \\
\hline Dental Service & 5 \\
\hline $\begin{array}{l}\text { Psychological assessment of learning difficulties/disability (offered by } \\
\text { counselling or disability service) }\end{array}$ & 5 \\
\hline $\begin{array}{l}\text { A dedicated service for international students (includes personal } \\
\text { counselling/psychological services) }\end{array}$ & 2 \\
\hline Other (please specify) & 2 \\
\hline
\end{tabular}




\section{Q36 Identify student services offered as part of counselling service responsibility or activities}

The majority of counselling services offered the following within their model of service delivery. There would appear to be five (5) key foci in service delivery demonstrated by counselling services in higher education in both New Zealand and Australia:

Counselling/psychological services for both local students $(\mathrm{N}=35)$ and international students $(\mathrm{N}=34)$, and counselling sensitive to the cultural needs of minority and indigenous (ATSI/Maori/PI) students $(\mathrm{N}=34)$.

Fewer counselling services offered case management of students referred to specialist services or external service providers $(\mathrm{N}=18)$ or Mental Health Educator or support services provided by a psychologist or nurse $(\mathrm{N}=11)$.

The responses provided by managers indicated a minority of counselling services providing adjunct services supportive of the student experience.

Table 22

\begin{tabular}{|c|c|}
\hline \multicolumn{2}{|c|}{ Student services offered as part of counselling service responsibility or activities } \\
\hline Answer Choices & $\begin{array}{l}\text { Number of } \\
\text { Institutions }\end{array}$ \\
\hline Counselling/psychological services for local students & 35 \\
\hline Counselling/psychological services for international students & 34 \\
\hline $\begin{array}{l}\text { Counselling/psychological services for minority/indigenous students } \\
\text { (ATSI/Maori/PI) }\end{array}$ & 34 \\
\hline $\begin{array}{l}\text { Case management of students referred to specialist services or external } \\
\text { service providers }\end{array}$ & 18 \\
\hline $\begin{array}{l}\text { Mental Health Educator or support services provided by a psychologist or } \\
\text { nurse }\end{array}$ & 11 \\
\hline Welfare services & 7 \\
\hline $\begin{array}{l}\text { Peer Mentoring Services (could be coordination or services such as mentor } \\
\text { training) }\end{array}$ & 7 \\
\hline $\begin{array}{l}\text { Reasonable educational accommodation adjustments for students with } \\
\text { disability or learning difficulties }\end{array}$ & 6 \\
\hline $\begin{array}{l}\text { Psychiatric Services (assessment/medication/treatment/longer term } \\
\text { psychotherapy) }\end{array}$ & 5 \\
\hline Careers counselling & 4 \\
\hline Academic skills/information literacy services & 4 \\
\hline Other (please specify) & 4 \\
\hline Budgeting and financial advice for students on low incomes & 3 \\
\hline Psychological assessment of learning difficulties/disability & 3 \\
\hline Academic advising services & 3 \\
\hline Transition to university/First Year Experience Coordination/Services & 3 \\
\hline Retention Programs (could be co-ordination or service provision) & 3 \\
\hline Student Volunteer Programs (could be coordination or services) & 3 \\
\hline Leadership development programs (could be coordination or services) & 3 \\
\hline No interest or low interest loans service & 2 \\
\hline Job readiness and employment preparation services & 2 \\
\hline $\begin{array}{l}\text { Learning engagement and enhancement services for specific cohorts } \\
\text { entering under special access programs (LSES, First in Family etc) }\end{array}$ & 2 \\
\hline English language support services & 1 \\
\hline
\end{tabular}

Comments provided by a responding manager identified the limits to therapeutic engagement:

"Longer-term psychotherapy occurs for a small minority where it involves ongoing risk issues and difficulty with engaging other services including crisis service." 
Another manager noted that the "case management position has been approved as a new initiative but not yet filled".

A manager highlighted the provision of "Crisis support, secondary consults, staff support (ad hoc when needed), counselling of staff, and offering Workshops (educational and therapeutic)".

Another manager identified that "Educators and Trainers" were included in the service activities.

Q37 Enrolment figures recorded at your institution across all campuses for the following cohorts (measured at 31 March 2017)

Five (5) survey responders did not make data available for this question.

Three (3) responding managers provided enrolment data as less than 10,000 at 31 March 2017.

Two (2) responding managers provided enrolment data as more than 10,000 and not higher than 20,000 at 31 March 2017.

Ten (10) responding managers provided enrolment data as more than 20,000 and not higher than 30,000 at 31 March 2017.

Four (4) responding managers provided enrolment data as more than 30,000 and not higher than 40,000 at 31 March 2017.

Two (2) responding managers provided enrolment data as more than 40,000 and not higher than 50,000 at 31 March 2017.

Three (3) responding managers provided enrolment data as more than 50,000 and not higher than 60,000 31 March 2017.

Three (3) responding managers provided enrolment data as more than 60,000 and not higher than 70,000 31 March 2017.

One (1) responding manager provided enrolment data as more than 70,000 at 31 March 2017.

Table 23

\begin{tabular}{|c|c|}
\hline Total Enrolment & Number of Institutions \\
\hline Data not available & 5 \\
\hline$<10,000$ & 3 \\
\hline$>10,000-20,000$ & 2 \\
\hline$>20,000-30,000$ & 10 \\
\hline$>30,000-40,000$ & 4 \\
\hline$>40,000-50,000$ & 2 \\
\hline$>50,000-60,000$ & 3 \\
\hline$>60,000-70,000$ & 3 \\
\hline$>70,000$ & 1 \\
\hline
\end{tabular}

Q38 Compared to 31 March 2016 enrolment figures, total student enrolment measured at 31 March 2017 decreased, increased or showed little variation?

Seventeen (17) responding managers reported an increase of enrolment comparing 2016 figures to 2017.

Six (6) responding managers reported a decrease of enrolment comparing 2016 figures to 2017. Nine (9) responding managers reported little variation of enrolment comparing 2016 figures to 2017. 
Table 24

\begin{tabular}{|c|c|}
\hline Enrolment changes & Number of Institutions \\
\hline Decrease & 6 \\
\hline Increased enrolment & 17 \\
\hline Little variation & 9 \\
\hline
\end{tabular}

\section{Q39 Cohort enrolment data}

Counselling Service managers were asked for data on the cohort enrolments at their institution. The demographics of enrolled cohorts vary considerably across the higher education institutions. These varying demographics can have considerable ramifications for counselling service operational planning. Approximately half of the managers provided data on enrolment totals. Many of the managers identified that it is difficult and for some, impossible, to access their institution's cohort data to guide service delivery planning and to anticipate demand for services. Even on basic cohort numbers many managers reported 'no available data'.

Data provided by managers for the enrolment figures for various cohorts and total number and fulltime effective (FTE) are provided below.

The full-time undergraduate coursework student enrolments ranged from $\mathrm{N}=3,792$ to 48,584 .

Table 25

\begin{tabular}{|c|c|}
\hline Full-time undergraduate coursework & Number of Institutions \\
\hline No available data & 15 \\
\hline $3,500-5,000$ & 2 \\
\hline$>5,000-7,000$ & 1 \\
\hline$>7,000-10,000$ & 3 \\
\hline$>10,000-20,000$ & 5 \\
\hline$>20,000-30,000$ & 3 \\
\hline$>30,000-40,000$ & 1 \\
\hline$>40,000$ & 1 \\
\hline
\end{tabular}

The effective fulltime enrolments (FTE) for full-time undergraduate coursework ranged from $\mathrm{N}=$ 4,118-43,636.

Table 26

\begin{tabular}{|c|c|}
\hline $\begin{array}{c}\text { FTE - Full-time undergraduate } \\
\text { coursework }\end{array}$ & Number of Institutions \\
\hline No available data & 15 \\
\hline $3,500-5,000$ & 2 \\
\hline$>5,000-7,000$ & 1 \\
\hline$>7,000-10,000$ & 3 \\
\hline$>10,000-20,000$ & 5 \\
\hline$>20,000-30,000$ & 1 \\
\hline$>30,000-40,000$ & 1 \\
\hline$>40,000$ & 1 \\
\hline
\end{tabular}


For Part-time Undergraduate Coursework the enrolment range reported was $\mathrm{N}=2,937$ to 14,732 .

Table 27

\begin{tabular}{|c|c|}
\hline $\begin{array}{c}\text { Part-time Undergraduate } \\
\text { Coursework }\end{array}$ & Number of Institutions \\
\hline No data available & 18 \\
\hline$>2000-5000$ & 3 \\
\hline$>5000-10000$ & 3 \\
\hline$>10000-15000$ & 4 \\
\hline
\end{tabular}

FTE Part-time Undergraduate Coursework enrolment: By error this data was not requested in survey.

For full-time postgraduate coursework enrolment the Range reported was $\mathrm{N}=815$ to 6,500 .

Table 28

\begin{tabular}{|c|c|}
\hline Full-time postgraduate coursework & Number of Institutions \\
\hline No data available & 16 \\
\hline$<1000-2000$ & 5 \\
\hline$>2000-5000$ & 5 \\
\hline$>5000-8000$ & 4 \\
\hline
\end{tabular}

Enrolment FTE Full-time postgraduate coursework

Range reported was $\mathrm{N}=815$ to 11,879 .

Table 29

\begin{tabular}{|c|c|}
\hline $\begin{array}{c}\text { FTE Full-time postgraduate } \\
\text { coursework }\end{array}$ & Number of Institutions \\
\hline No data available & 16 \\
\hline$<1000-2000$ & 7 \\
\hline$>2000-5000$ & 6 \\
\hline$>5000-8000$ & 0 \\
\hline$>8000$ & 1 \\
\hline
\end{tabular}

Enrolment Part-time Postgraduate Coursework: By error data not requested in Survey

FTE Part-time Postgraduate Coursework Enrolment Range 438-4701

Table 30

\begin{tabular}{|c|c|}
\hline $\begin{array}{c}\text { FTE Part-time Postgraduate } \\
\text { Coursework }\end{array}$ & Number of Institutions \\
\hline No data available & 19 \\
\hline$<2000$ & 8 \\
\hline$>2000-6000$ & 2 \\
\hline
\end{tabular}

Full-time postgraduate Research Enrolment Range reported was $\mathrm{N}=165$ to 2,852.

Table 31

\begin{tabular}{|c|c|}
\hline Full-time postgraduate Research & Number of Institutions \\
\hline No data available & 17 \\
\hline$<200-500$ & 4 \\
\hline$>500-1000$ & 4 \\
\hline$>1000-2000$ & 3 \\
\hline$>2000$ & 2 \\
\hline
\end{tabular}


FTE Full-time postgraduate Research Enrolment range reported was N $=165$ to 3282 .

Table 32

\begin{tabular}{|c|c|}
\hline $\begin{array}{c}\text { FTE Full-time postgraduate } \\
\text { Research }\end{array}$ & Number of Institutions \\
\hline No data available & 17 \\
\hline$<200-500$ & 4 \\
\hline$>500-1000$ & 3 \\
\hline$>1000-2000$ & 4 \\
\hline$>2000$ & 2 \\
\hline
\end{tabular}

FTE Part-time postgraduate research students Enrolment range reported was $\mathrm{N}=77$ to 456 .

Table 33

\begin{tabular}{|c|c|}
\hline $\begin{array}{c}\text { FTE Part-time postgraduate } \\
\text { research students }\end{array}$ & Number of Institutions \\
\hline No data available & 20 \\
\hline$<1000$ & 9 \\
\hline
\end{tabular}

Q40 International and Local student enrolments all data

Total enrolments reported for international and local students

Table 34

\begin{tabular}{|l|c|}
\hline \multicolumn{1}{|c|}{ International and Local enrolments } & Total Number \\
\hline $\begin{array}{l}\text { International Students (on student } \\
\text { visas) - Total head count }\end{array}$ & 168,848 \\
\hline $\begin{array}{l}\text { Local students (includes permanent } \\
\text { residents) - Total head count }\end{array}$ & 517,779 \\
\hline Total head count & 686,627
\end{tabular}

International Students (on student visas): Range reported was $\mathrm{N}=1,000$ to 29,477

Table 35

\begin{tabular}{|c|c|}
\hline $\begin{array}{c}\text { International Students (on student } \\
\text { visas) }\end{array}$ & Number of Institutions \\
\hline No data available & 9 \\
\hline $1000-2000$ & 4 \\
\hline$>2000-3000$ & 3 \\
\hline$>3000-4000$ & 2 \\
\hline$>4000-10000$ & 6 \\
\hline$>10000-20000$ & 3 \\
\hline$>20000-30000$ & 2 \\
\hline
\end{tabular}

Local students (includes permanent residents): Enrolment Range reported N =2,000 to 44,330.

Table 36

\begin{tabular}{|c|c|}
\hline $\begin{array}{c}\text { Local students (includes permanent } \\
\text { residents) }\end{array}$ & Number of Institutions \\
\hline No data available & 10 \\
\hline $2000-3000$ & 1 \\
\hline$>3000-20000$ & 5 \\
\hline$>20000-30000$ & 5 \\
\hline$>30000-40000$ & 5 \\
\hline$>40000$ & 3 \\
\hline
\end{tabular}


Combined responses for cohort enrolled numbers by diversity groupings

Table 37

\begin{tabular}{|l|c|}
\multicolumn{1}{|c|}{ Student Enrolment - Total Head Count } & Total Number \\
\hline Students who identify as indigenous (e.g. ATSI, Maori, Pacific Islander) & 18,328 \\
\hline Students identified as low SES & 59,613 \\
\hline Students registered for disability learning provisions & 24,062 \\
\hline Students for whom English is not their first language & 72,005 \\
\hline Students who moved from a rural area, another city, another state & 13,441 \\
\hline $\begin{array}{l}\text { Students who are returning to education after more than one year of no } \\
\text { study }\end{array}$ & 22,186 \\
\hline Students who are transferring with credit from another institution & 14,352
\end{tabular}

Students who identify as indigenous (e.g. ATSI, Maori, Pacific Islander): Enrolment Range reported was $\mathrm{N}=3$ to 5,229 .

Table 38

\begin{tabular}{|c|c|}
\hline $\begin{array}{c}\text { Students who identify as indigenous } \\
\text { (e.g. ATSI, Maori, Pacific Islander) }\end{array}$ & Number of Institutions \\
\hline No data available & 12 \\
\hline $3-100$ & 1 \\
\hline$>100-200$ & 0 \\
\hline$>200-300$ & 4 \\
\hline$>300-400$ & 2 \\
\hline$>400-500$ & 2 \\
\hline$>500-600$ & 1 \\
\hline$>600-700$ & 0 \\
\hline$>700-800$ & 3 \\
\hline$>800-900$ & 2 \\
\hline$>900-1000$ & 0 \\
\hline$>1000-2000$ & 1 \\
\hline$>2000-3000$ & 0 \\
\hline$>3000-4000$ & 1 \\
\hline$>4000$ & 1 \\
\hline
\end{tabular}

Students identified as low SES: Enrolment Range reported was N $=1,200$ to 19,370.

Table 39

Table 39
\begin{tabular}{|c|c|}
\hline Students identified as low SES & Number of Institutions \\
\hline No data available & 19 \\
\hline $1000-2000$ & 2 \\
\hline$>2000-3000$ & 0 \\
\hline$>3000-4000$ & 1 \\
\hline$>4000-5000$ & 2 \\
\hline$>5000-6000$ & 1 \\
\hline$>6000-7000$ & 3 \\
\hline$>7000-20000$ & 1 \\
\hline
\end{tabular}


Students registered for disability learning provisions: Enrolment Range reported was $\mathrm{N}=100$ to 2,769 .

Table 40

\begin{tabular}{|c|c|}
\hline $\begin{array}{c}\text { Students registered for disability } \\
\text { learning provisions }\end{array}$ & Number of Institutions \\
\hline No available data & 12 \\
\hline $1-100$ & 2 \\
\hline$>100-1000$ & 2 \\
\hline$>1000-2000$ & 6 \\
\hline$>2000-3000$ & 4 \\
\hline
\end{tabular}

Students for whom English is not their first language: Enrolment Range reported was $\mathrm{N}=1,000$ to 17,606.

Table 41

\begin{tabular}{|c|c|}
\hline $\begin{array}{l}\text { Students for whom English is not } \\
\text { their first language }\end{array}$ & Number of Institutions \\
\hline Data not available & 14 \\
\hline $1000-2000$ & 2 \\
\hline$>2000-3000$ & 2 \\
\hline$>3000-4000$ & 1 \\
\hline$>4000-5000$ & 0 \\
\hline$>5000-6000$ & 1 \\
\hline$>6000$ & 1 \\
\hline
\end{tabular}

Students who moved from a rural area, another city, another state: Enrolment Range reported was N $=1,000$ to 5,592 .

Table 42

\begin{tabular}{|c|c|}
\hline $\begin{array}{c}\text { Students who moved from a rural } \\
\text { area, another city, another state }\end{array}$ & Number of Institutions \\
\hline Data not available & 24 \\
\hline $1000-2000$ & 1 \\
\hline$>2000-3000$ & 1 \\
\hline$>3000-4000$ & 0 \\
\hline$>4000-5000$ & 1 \\
\hline$>5000$ & 1 \\
\hline
\end{tabular}

Students who are returning to education after more than one year of no study: Enrolment Range reported was $\mathrm{N}=371$ to 19,288 .

Table 43

\begin{tabular}{|c|c|}
\hline $\begin{array}{c}\text { Students who are returning to } \\
\text { education after more than one year } \\
\text { of no study }\end{array}$ & Number of Institutions \\
\hline No data available & 23 \\
\hline$<1000$ & 1 \\
\hline$>1000-2,000$ & 2 \\
\hline$>2000-19,288$ & 1 \\
\hline
\end{tabular}


Students who are transferring with credit from another institution: Enrolment Range reported was N $=859$ to 5,070 .

Table 44

\begin{tabular}{|c|c|}
\hline $\begin{array}{c}\text { Students who are transferring with } \\
\text { credit from another institution }\end{array}$ & Number of Institutions \\
\hline Data not available & 22 \\
\hline$<1000$ & 1 \\
\hline$>1000-2000$ & 1 \\
\hline$>2000-3000$ & 1 \\
\hline$>3000-4000$ & 0 \\
\hline$>4000-5000$ & 1 \\
\hline$>5000$ & 1 \\
\hline
\end{tabular}

Q41 Enrolled Cohort x Gender

Ten (10) institutions indicated that data was not available.

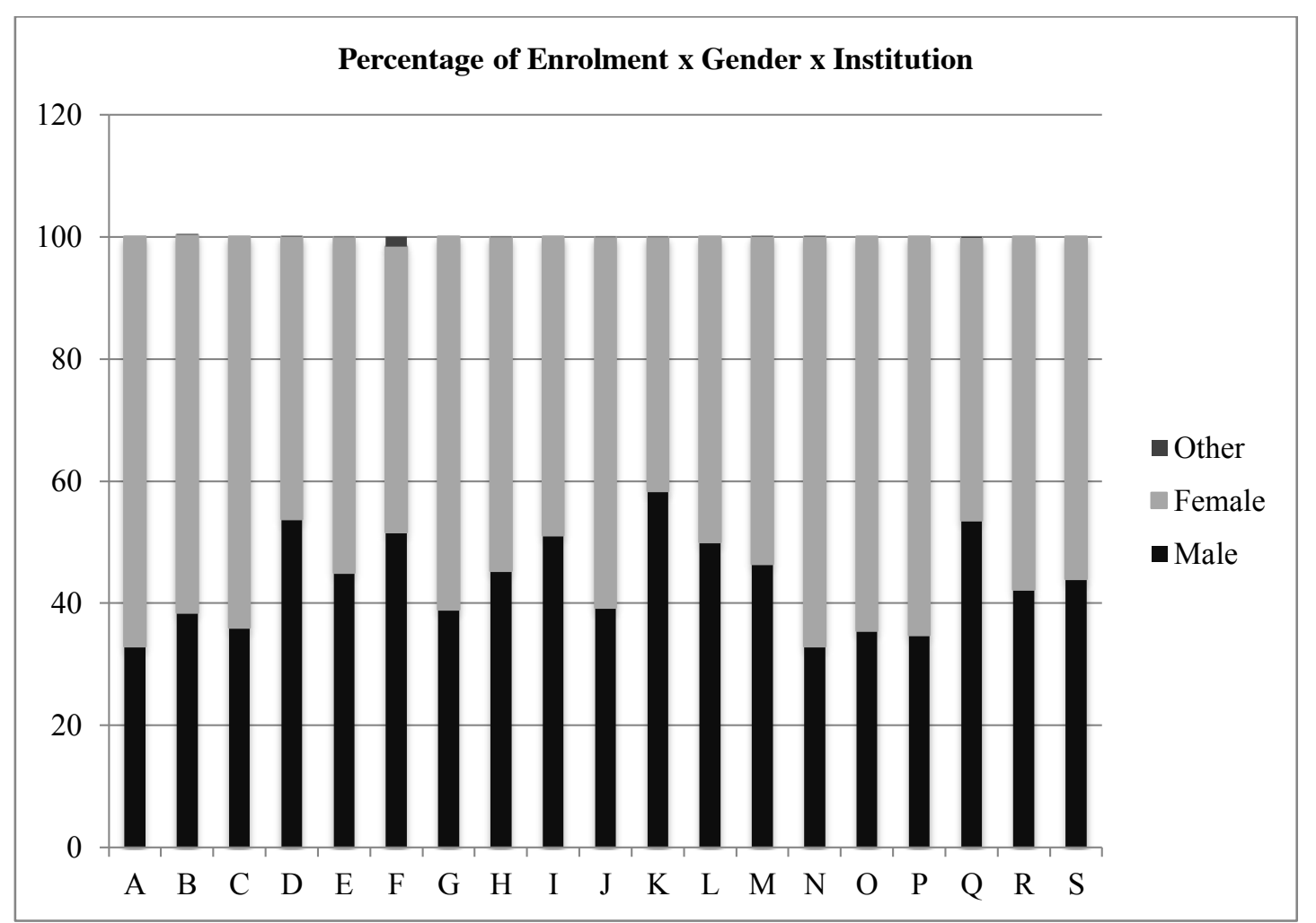

Figure 15

Q42 Percentage of enrolments:

a) Students aged less than 18 yrs: Range reported was $0.08 \%$ to $6 \%$.

Table 45

\begin{tabular}{|c|c|}
\hline Percentage of enrolment under 18 yrs & Number of Institutions \\
\hline No available data & 19 \\
\hline$<1 \%$ & 5 \\
\hline $1-2 \%$ & 2 \\
\hline$>2 \%$ & 3 \\
\hline
\end{tabular}

b) Enrolled students living in halls of residence or colleges on campus or in accommodation associated with your institution: Range reported was $1 \%$ to $30 \%$. 
Table 46

\begin{tabular}{|c|c|}
\hline $\begin{array}{c}\text { Percentage of students in on campus } \\
\text { residences }\end{array}$ & Number of Institutions \\
\hline No available data & 18 \\
\hline $1-3 \%$ & 5 \\
\hline$>3-6 \%$ & 4 \\
\hline$>6-15 \%$ & 1 \\
\hline$>15-30 \%$ & 1 \\
\hline
\end{tabular}

c) Enrolled students who are first in their immediate family to attend post-secondary education/university: Range reported was $24.4 \%$ to $36 \%$.

Table 47

\begin{tabular}{|c|c|}
\hline $\begin{array}{c}\text { Enrolled students who are first in their } \\
\text { immediate family to attend post-secondary } \\
\text { education/university }\end{array}$ & Number of Institutions \\
\hline No available data & 27 \\
\hline $24.40 \%$ & 1 \\
\hline $36 \%$ & 1 \\
\hline
\end{tabular}

d) Enrolled students who are commencing (first year in program): Range reported was $0.37 \%$ to $75 \%$.

Table 48

\begin{tabular}{|c|c|}
\hline $\begin{array}{c}\text { Enrolled students are commencing } \\
\text { (first year in program) }\end{array}$ & Number of Institutions \\
\hline No data available & 13 \\
\hline$<10$ & 1 \\
\hline$>10-20$ & 4 \\
\hline$>20-30$ & 3 \\
\hline$>30-40$ & 3 \\
\hline$>40-50$ & 2 \\
\hline$>50$ & 2 \\
\hline
\end{tabular}

Q43 Is your institution primarily known as a public or private college/university?

The majority of managers (93.9\%) worked in a public university.

Table 49

\begin{tabular}{|c|c|}
\hline Answer Choices & Number of Institutions \\
\hline Public & 31 \\
\hline Private & 2 \\
\hline
\end{tabular}

Q44 Institution provides learning via flexible/distance and/or on campus classes:

The majority of institutions $(\mathrm{N}=38)$ provided course via flexible delivery and on campus attendance.

Table 50

\begin{tabular}{|l|c|}
\hline \multicolumn{1}{|c|}{ Learning via flexible/distance and/or on campus classes } & Number of Institutions \\
\hline Flexible delivery/distance options only & 2 \\
\hline Blend of flexible delivery, distance and on campus attendance & 15 \\
\hline $\begin{array}{l}\text { Primarily on campus attendance with some flexible access via use of } \\
\text { online teaching environment }\end{array}$ & 2 \\
\hline Other (please specify) & \multicolumn{1}{|c|}{2} \\
\hline
\end{tabular}

Comments provided were not related to the modes of learning and therefore are not included in the report.

Q45 Operation of 'onshore' campuses

The range of 'onshore' campuses varied from zero for one institution that only offered online 
courses to more than 10 campuses.

Table 51

\begin{tabular}{|c|c|}
\hline Operate 'onshore' campuses & Number of Institutions \\
\hline none - all virtual/remote & 1 \\
\hline 1 & 0 \\
\hline 2 & 3 \\
\hline 3 & 9 \\
\hline 4 & 7 \\
\hline 5 & 1 \\
\hline 6 & 4 \\
\hline 7 & 3 \\
\hline 8 & 1 \\
\hline 9 & 0 \\
\hline 10 & 0 \\
\hline$>10$ & 2 \\
\hline
\end{tabular}

Q46 Change in the number of 'on shore' campuses in 2017

The majority of institutions $(\mathrm{N}=26)$ had not changed the number of campuses provided 'onshore' during 2017. Three (3) institutions had increased, and two (2) had decreased the number of 'onshore' campuses.

Table 52

\begin{tabular}{|c|c|}
\hline Change in Onshore Campuses & Number of Institutions \\
\hline No change & 26 \\
\hline Increased & 3 \\
\hline Decreased & 2 \\
\hline Other (please specify) & 3 \\
\hline
\end{tabular}

Other specified:

- Though I only manage 1 campus

- Related to 44 - 2 on shore campus with 3 teaching locations

- We have a Sydney "campus" and regional study centres

Q47 Operation of 'offshore' campuses

The majority of managers $(\mathrm{N}=19)$ reported that offshore enrolments were managed via online course delivery. The number of offshore campuses reported ranged from one (1) reported by eight (8) managers to five (5) reported by one (1) manager.

Table 53

\begin{tabular}{|c|c|}
\hline Operation of Off Shore Campuses & Number of Institutions \\
\hline none - all virtual/remote & 19 \\
\hline 1 & 8 \\
\hline 2 & 0 \\
\hline 3 & 1 \\
\hline 4 & 2 \\
\hline 5 & 1 \\
\hline$>5$ & 0 \\
\hline
\end{tabular}

Q48 Change in the number of 'off shore' campuses in 2017

Almost all managers $(\mathrm{N}=30)$ reported no change in the number of offshore campuses during 2017. 


\begin{tabular}{|c|c|}
\hline $\begin{array}{c}\text { Change in Number of Off Shore } \\
\text { Campuses in 2017 }\end{array}$ & Number of Institutions \\
\hline No change & 30 \\
\hline Increased & 1 \\
\hline Decreased & 0 \\
\hline
\end{tabular}

Q49 Delivery of comparable services by the counselling service to all students enrolled with the institution irrespective of where they are studying

Most managers $(\mathrm{N}=22)$ reported that all students were offered comparable services irrespective of campus or enrolment type. Four (4) managers reported that only students attending on-shore campuses receive comparable services. One manager reported under 'other' that all students were offered the comparable service except those in overseas locations. Four managers $(\mathrm{N}=4)$ reported that only students attending specific on-shore campuses received comparable services. Two (2) managers reported that only students attending on-shore and specific offshore campuses received comparable services. Under 'Other', one (1) manager stated that the service was primarily accessed in person even though other options were theoretically available. Another manager stated that distance students were provided with Skype counselling sessions only if agreed by the counsellor.

Table 55

\begin{tabular}{|l|c|}
\hline \multicolumn{1}{|c|}{ Delivery of comparable services } \\
\hline \multicolumn{1}{|c|}{ Answer Choices } & Number of Institutions \\
\hline $\begin{array}{l}\text { Yes - all students are offered comparable services irrespective of campus or } \\
\text { enrolment type }\end{array}$ & 0 \\
\hline $\begin{array}{l}\text { No - students enrolled in only online programs are not currently eligible for } \\
\text { the same services }\end{array}$ & \\
\hline $\begin{array}{l}\text { No - only students attending on-shore campuses receive comparable } \\
\text { services }\end{array}$ & 4 \\
\hline $\begin{array}{l}\text { No - only students attending on-shore and specific off-shore campuses } \\
\text { receive comparable services }\end{array}$ & 2 \\
\hline $\begin{array}{l}\text { No - only students attending specific on-shore campuses receive comparable } \\
\text { services }\end{array}$ & \\
\hline Other (please specify) & 4 \\
\hline
\end{tabular}

Other specified:

- In theory yes, but in practice access is primarily for in person.

- Though Distance students may not find it as easy to obtain or to attend on-site campus provided counselling. Skype counselling depends on practitioner's preference.

- All students offered the comparable service except those in overseas locations

Q50 Enrolled students who have access to a service dedicated to the provision of counselling and psychological assistance

Half of the managers indicated that service delivery was provided to students while they were 'off shore' and an equal number stated that they did not provide services to students while they were 'off shore' due to limitations imposed on psychological practice by country legislation. 
Table 56

\begin{tabular}{|l|c|}
\hline \multicolumn{2}{|c|}{$\begin{array}{c}\text { Enrolled students with access to a service dedicated to the provision of counselling and } \\
\text { psychological assistance }\end{array}$} \\
\hline \multicolumn{1}{|c|}{ Answer Choices } & Number of Institutions \\
\hline $\begin{array}{l}\text { All enrolled students (including those requesting services while in another } \\
\text { country or studying on exchange with another university). }\end{array}$ & 16 \\
\hline $\begin{array}{l}\text { Only enrolled students located in the same country as the counselling } \\
\text { service due to limitations imposed on psychological practice by country } \\
\text { legislation (i.e. excluding those requesting services while in another } \\
\text { country). }\end{array}$ & 16 \\
\hline Other (please specify) & 1 \\
\hline
\end{tabular}

Other specified:

Though if a student has already received a service in NZ \& is then offshore temporarily, we may provide through Skype/Wechat.

Q51 Does your institution have a comprehensive emergency/critical incident response plan that includes expertise provided by counselling service staff?

The majority of managers $(\mathrm{N}=23)$ reported that their institution had a comprehensive emergency/critical incident plan. Two (2) managers were uncertain and eight (8) reported that their institution did not have a comprehensive emergency/critical incident plan.

Table 57

\begin{tabular}{|c|c|}
\hline $\begin{array}{c}\text { Comprehensive emergency/critical incident response plan that includes } \\
\text { expertise provided by counselling service staff }\end{array}$ \\
\hline Answer Choices & Number of Institutions \\
\hline No & 8 \\
\hline Yes & 23 \\
\hline Uncertain & 2 \\
\hline
\end{tabular}

Q52 How much confidence do you feel in your institution's preparedness and capacity to respond to a critical event on campus that threatens the safety of one or more persons?

Almost all managers $(\mathrm{N}=29)$ felt moderately $(\mathrm{N}=18)$ or very confident $(\mathrm{N}=11)$ in their institution's preparedness and capacity to respond to a critical event on campus that threatens the safety of one or more persons.

Table 58

Confidence in institution's preparedness and capacity to respond to a critical event on campus

\begin{tabular}{|c|c|}
\hline Answer Choices & Number of Institutions \\
\hline Not at all confident & 3 \\
\hline Moderately confident & 18 \\
\hline Very confident & 11 \\
\hline
\end{tabular}

Additional comments further identified that some managers were very aware of preparations made for responses to emergencies and critical incidents with prior events providing clear evidence of preparation and post-event planning for future events. One comment expressed concern that the counselling team whilst part of an emergency response had no visible input to the planning.

Comments:

- There is a plan. It seems to go along okay initially. There isn't visible input from Counselling service re: expertise but there is reliance on counselling support for those impacted.

- We are not practiced in responding to a people safety issue, though we are confident in our capacity to respond to a generic weather/buildings to a related emergency one. 
- We have had several significant earthquakes in recent years which have severely damaged uni buildings and closed the uni for periods, well prepared for more

- Crisis management team in place

- Annual scenario practice by high level response team has refined practices and policy over the last decade.

Q53 Does your institution have a mental health policy/framework/strategy?

Forty-six percent $(46 \%)$ of managers reported that their institution had a mental health policy/framework/strategy.

Table 59

\begin{tabular}{|c|c|}
\hline \multicolumn{2}{|c|}{ Have a mental health policy/framework/strategy } \\
\hline Answer Choices & Number of Institutions \\
\hline Yes & 12 \\
\hline No & 14 \\
\hline
\end{tabular}

Managers were asked to provide a url for the document.

Responses:

- http://www.anu.edu.au/about/strategic-planning/mental-health-strategy

- In development based on this framework: http://unistudentwellbeing.edu.au/framework/.

- http://www.flinders.edu.au/ppmanual/student/student-mental-health-wellbeing.cfm

Other comments:

- Victoria- A health Promoting University 2017 - Victoria Mental Health and Wellbeing Plan 2014-2017.

- Draft at this stage.

- Undertaking this.

Q54 Is the mental health policy/framework/strategy 'stand-alone'?

Approximately $50 \%$ of managers indicated that the mental health policy/framework/strategy was 'stand-alone'.

Table 60

\begin{tabular}{|c|c|}
\hline \multicolumn{2}{|c|}{ Mental health policy/framework/strategy is 'stand-alone' } \\
\hline Answer Choices & Number of Institutions \\
\hline Yes & 12 \\
\hline No & 11 \\
\hline
\end{tabular}

Managers were asked to explain the relationship of the mental health policy/framework/strategy to other policies/action plans etc.

Comments:

- N/A

- Still being developed.

- As this is developed, the framework will sit above all other policies across the institution. Yet to be realised in practice!

- There is also a Mental Health and Wellbeing Action Plan. 
- Integrated into critical response framework.

Q55 Is your institution officially a "health promoting university"? (i.e. joined the Healthy Universities Network or joined a Health Promoting University Network and/or signed the Okanagan Charter).

Approximately $50 \%$ of managers reported that their institution was officially a 'health promoting university'.

Table 61

\begin{tabular}{|c|c|}
\hline \multicolumn{2}{|c|}{ "health promoting university" } \\
\hline Answer Choices & Number of Institutions \\
\hline Yes & 12 \\
\hline No & 18 \\
\hline
\end{tabular}

Q56 Is your institution informally adopting a "health promoting university/healthy universities" approach endorsed by senior leadership?

Approximately $70 \%$ of managers reported that their institution was informally adopting a "health promoting university/healthy universities" approach with endorsement from senior leadership.

Table 62

\begin{tabular}{|c|c|}
\hline $\begin{array}{c}\text { Institution informally adopting a "health promoting university/healthy } \\
\text { universities" approach endorsed by senior leadership }\end{array}$ \\
\hline Answer Choices & Number of Institutions \\
\hline Yes & 19 \\
\hline No & 12 \\
\hline
\end{tabular}

Q57 Beyond the provision of 1-1 counselling and psychological services, which of the following areas of activity did the mission, role and functions of your service specifically target in 2017? Tick all that apply.

Strategies to enhance overall student wellbeing were the most common area of service activity beyond 1-1 counselling and psychological services.

Rated in relation to frequency of response the following seven (7) activities were the focus of at least eighteen (18) services (in addition to 1-1 counselling).

1. Student development - life skills, interpersonal skills, psychological resilience etc

2. Institutional responsiveness to mental health issues

3. Contribution to the management of inappropriate/difficult/challenging student behaviour

4. Safer community interventions

5. Input to student policies and procedures

6. Early identification and intervention for risk of self harm or other harm in the student cohort

7. Contribution to the response to student misconduct complaints 
Table 63

Beyond the provision of 1-1 counselling and psychological services - areas of activity did the mission, role and functions of your service specifically target in 2017

\begin{tabular}{|l|c|}
\hline \multicolumn{1}{|c|}{ Answer Choices } & $\begin{array}{c}\text { Number of } \\
\text { Institutions }\end{array}$ \\
\hline Strategies to enhance overall student well being & 27 \\
\hline Student development - life skills, interpersonal skills, psychological resilience etc & 25 \\
\hline Institutional responsiveness to mental health issues & 24 \\
\hline Contribution to the management of inappropriate/difficult/challenging student behaviour & 23 \\
\hline Safer community interventions & 20 \\
\hline Input to student policies and procedures & 19 \\
\hline Early identification and intervention for risk of self harm or other harm in the student cohort & 18 \\
\hline Contribution to the response to student misconduct complaints & 18 \\
\hline Enhancing student academic performance & 14 \\
\hline Strategies to reduce student attrition/enhance student retention & 14 \\
\hline Student experience of campus life & 14 \\
\hline Equity and access support for students & 12 \\
\hline Recognition and response to diversity in the student population & 12 \\
\hline Student Mental Health Institution Strategy & 11 \\
\hline Student academic engagement & 11 \\
\hline Healthy universities interventions & 9 \\
\hline Other (please specify) & 1 \\
\hline
\end{tabular}

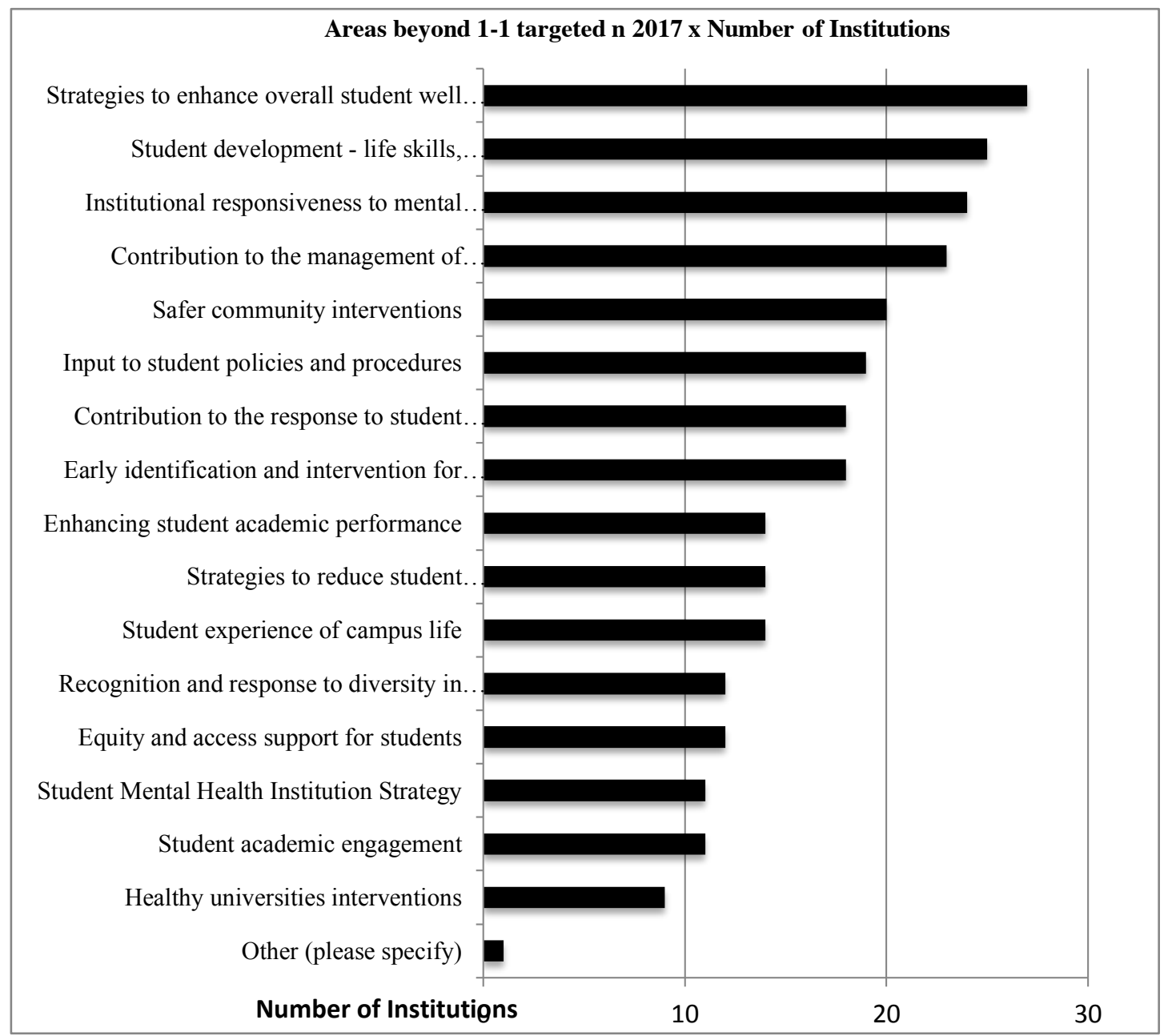

Figure 16

Journal of the Australian and New Zealand Student Services Association:

Volume 27, Issue 1 
Other specified:

Response to sexual assault within the organisation.

Q58 Which of the following does the service provide? Tick all that apply.

Twelve (12) activities within service provision were nominated by at least eighteen (18) managers.

The twelve most common activities beyond 1-1 services were:

- Consultation on student related issues with institution staff.

- Consultation on student related issues with college or halls of residence staff.

- Training programs for 'residential advisors' in colleges or halls of residence.

- Mental Health First Aid or similar workshop that educates staff or students about mental health/illness, 'at risk' behaviours and self-harm and suicidality.

- Program of psycho-educational workshops.

- Website with student focused resources.

- Faculty requested workshops outside class schedules.

- After hours workshops at colleges or halls of residence.

- Consultation on student related issues with staff in organisations affiliated with your institutions.

- Within curriculum 'guest' lectures.

- Specific programs for different cohort level (undergraduate / postgraduate /research).

- Consultation on student related issues with parents of students.

Table 64

\begin{tabular}{|l|c|}
\hline \multicolumn{1}{|c|}{ Answer Choices } & $\begin{array}{c}\text { Number of } \\
\text { Institutions }\end{array}$ \\
\hline & 30 \\
\hline Consultation on student related issues with institution staff & 30 \\
\hline Consultation on student related issues with college or halls of residence staff & 28 \\
\hline Training programs for 'residential advisors' in colleges or halls of residence & 28 \\
\hline $\begin{array}{l}\text { Mental Health First Aid or similar workshop that educates staff or students about mental } \\
\text { health/illness, 'at risk' behaviours and self-harm and suicidality. }\end{array}$ & 26 \\
\hline Program of psycho-educational workshops & 26 \\
\hline Website with student focused resources & 23 \\
\hline Faculty requested workshops outside class schedules & 20 \\
\hline After hours workshops at colleges or halls of residence & 19 \\
\hline Consultation on student related issues with staff in organisations affiliated with your institutions & 19 \\
\hline Within curriculum 'guest' lectures & 18 \\
\hline Specific programs for different cohort level (undergraduate / postgraduate /research) & 18 \\
\hline Consultation on student related issues with parents of students & 16 \\
\hline Training programs for students involved in volunteer activities & 15 \\
\hline Therapeutically focused group programs & 13 \\
\hline Specific orientation program for first year students & 11 \\
\hline Specific workshops for advanced year students & 9 \\
\hline Consultation on student related issues with staff at other institutions supported via an MOU & 7 \\
\hline Peer mentoring programs & 7 \\
\hline Courses to promote psychological and/or personal development in an online teaching & \\
\hline
\end{tabular}

Journal of the Australian and New Zealand Student Services Association:

Volume 27, Issue 1

108 


\begin{tabular}{|l|c|}
\hline \multicolumn{1}{|c|}{ Services provided } \\
\hline \multicolumn{1}{|c|}{ Answer Choices } & $\begin{array}{c}\text { Number of } \\
\text { Institutions }\end{array}$ \\
\hline environment & 7 \\
\hline Website with interactive capability supporting dynamic engagement with students & 7 \\
\hline Programs that aim to educate students on their use of alcohol and other drugs & 8 \\
\hline Specific programs for academically at risk students & 5 \\
\hline Other (please specify) & 1 \\
\hline $\begin{array}{l}\text { Programs that target internet overuse, e.g. behaviours related to harmful levels of gambling or } \\
\text { gaming via the internet }\end{array}$ & \\
\hline
\end{tabular}

Other activities specified:

- Workshops for staff on Psychological First Aid, Dealing with difficult behaviour of students, mental health and well-being awareness and health promotion events.

- Training Program for Staff: First Response: Student in Distress (Training to equip staff with skills to response to a student who is in distress).

- UWA has a separate Health Promotions Unit that does pro-active education and health promotions work.

- Online treatment programs for students.

- Programs regarding consent and sexual assault. 


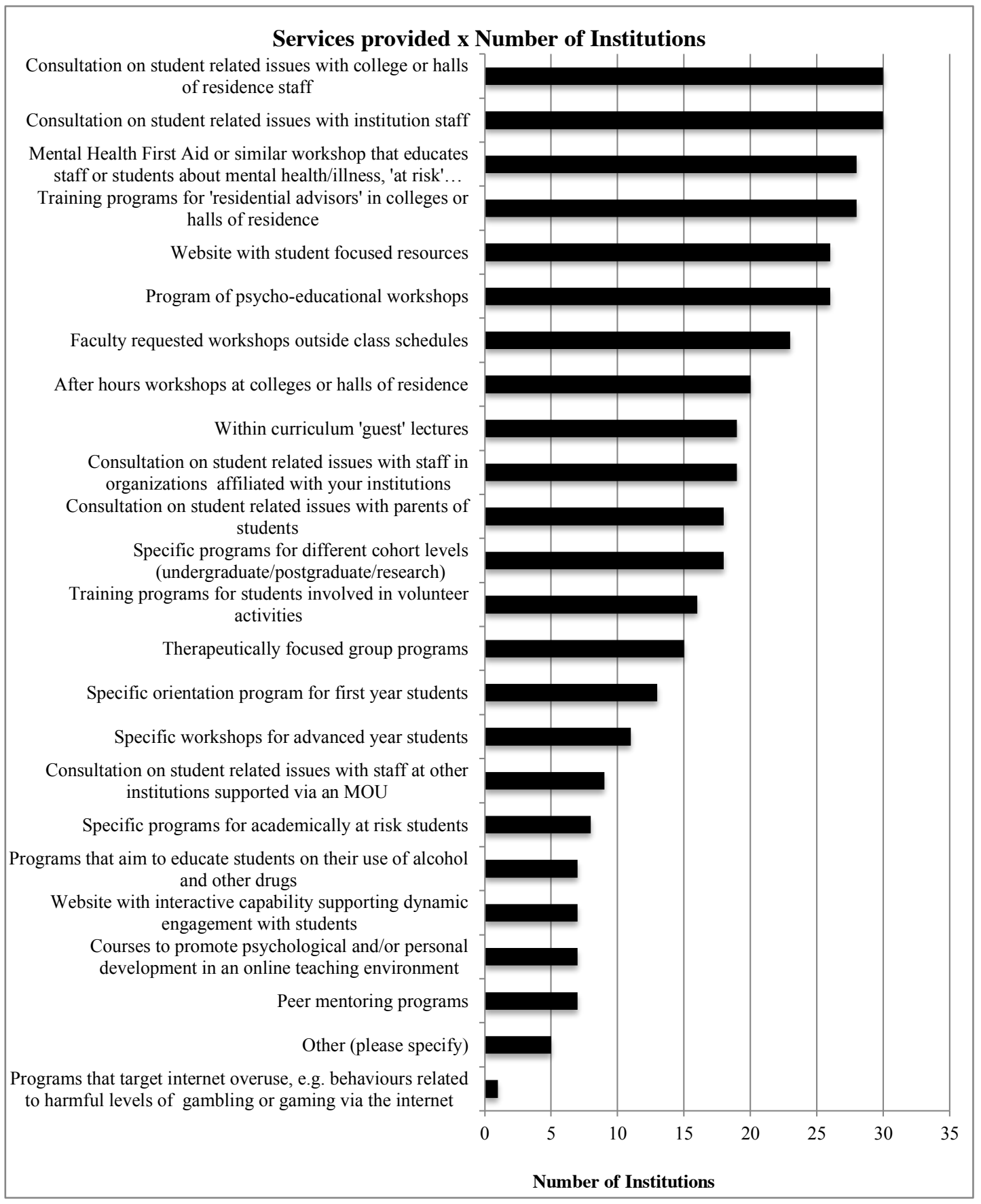

Figure 17

Q59 Which of the following quality assurance activities are conducted on a regular basis? Tick all that apply.

Two (2) quality assurance activities were identified by more than 20 managers. These were:

- Regular review of client needs and emerging student issues.

- Mapping of service strategic goals and operational plans against the institutional strategic plans and relevant key performance target/indicators. 
Table 65

\begin{tabular}{|l|c|}
\hline \multicolumn{1}{|c|}{ Quality Assurance Activities } \\
\hline \multicolumn{1}{|c|}{ Answer Choices } & $\begin{array}{c}\text { Number of } \\
\text { Institutions }\end{array}$ \\
\hline Regular review of client needs and emerging student issues & 25 \\
\hline $\begin{array}{l}\text { Mapping of service strategic goals and operational plans against the institutional strategic } \\
\text { plans and relevant key performance target/indicators }\end{array}$ & 21 \\
\hline Regular assessment of service efficacy and achievement & 17 \\
\hline Occasional formal service review & 17 \\
\hline Regular measurement of client satisfaction & 16 \\
\hline Regular review of service values, practices and procedures & 15 \\
\hline Regular use of feedback mechanisms & 13 \\
\hline Routine use of outcome measures & 12 \\
\hline Undertaking research pertinent to service delivery & 12 \\
\hline $\begin{array}{l}\text { Presentations and/or publications by staff on clinical issues, student needs and emerging } \\
\text { issues, service delivery effectiveness and service evaluation }\end{array}$ & 8 \\
\hline Other (please specify) & 2 \\
\hline None of the above & 0 \\
\hline
\end{tabular}

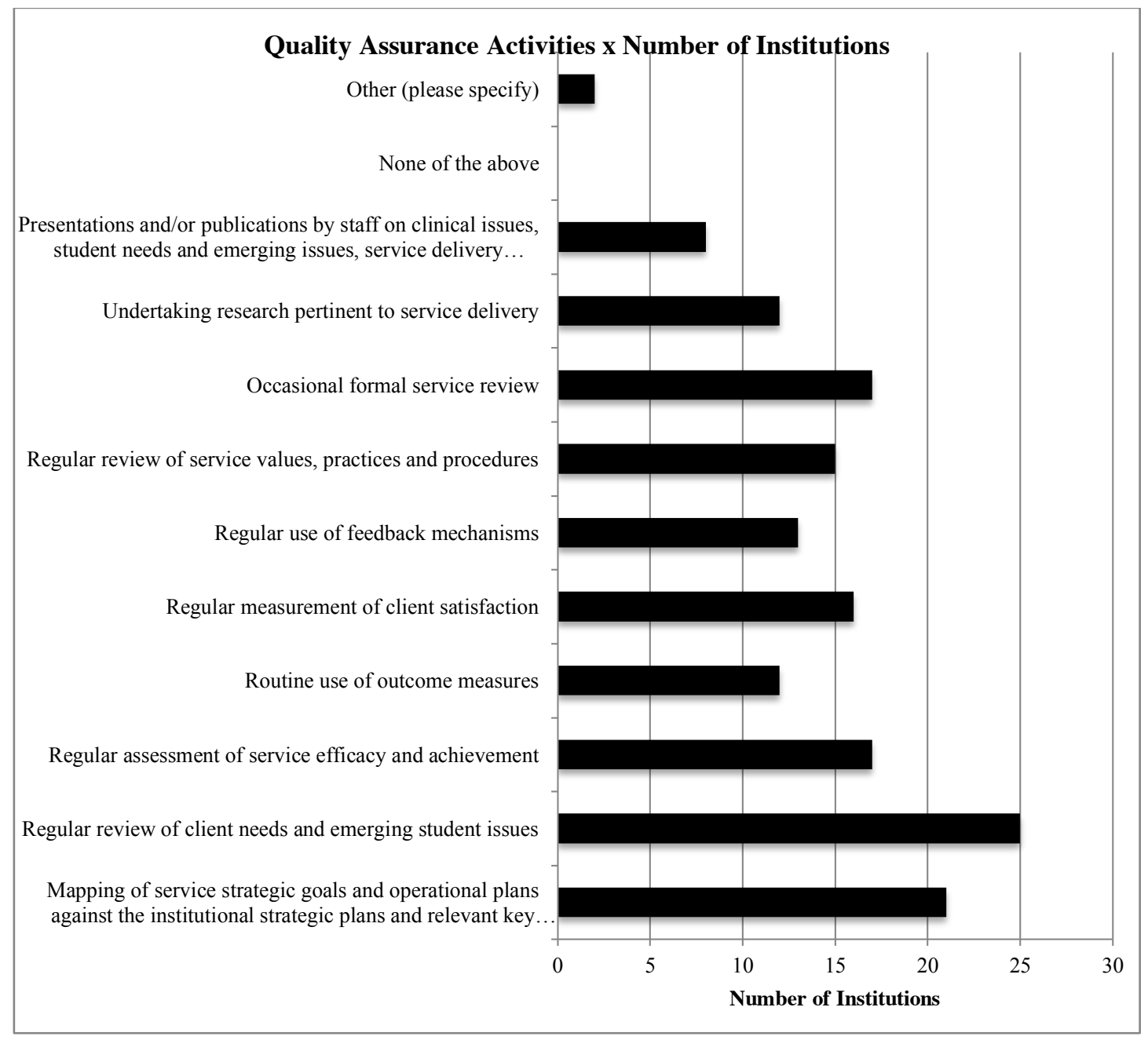

Figure 18 
Other specified:

Measurement of client satisfaction, client feedback - once per semester.

Annual client feedback questionnaires.

Q60 Which of the following clinical supervision/consultations or supervisory activities occur in the service? Tick any that have been utilised by service staff in the preceding 18 months.

There were four (4) clinical supervision/consultations or supervisory activities identified by at least 20 managers. These were:

- Ad-hoc case discussion with other team member as required.

- Peer consultation at least 2 hours per month (small group).

- Peer consultation with senior team member as needed.

- Individual supervision for at least 1 hour per month (with senior team member).

Table 66

\begin{tabular}{|l|c|}
\hline \multicolumn{1}{|c|}{ Clinical supervision/consultations or supervisory activities } \\
\hline \multicolumn{1}{|c|}{ Answer Choices } & $\begin{array}{c}\text { Number of } \\
\text { Institutions }\end{array}$ \\
\hline Ad-hoc case discussion with other team member as required & 32 \\
\hline Peer consultation at least 2 hours per month (small group) & 28 \\
\hline Peer consultation with senior team member as needed & 28 \\
\hline Individual supervision for at least 1 hour per month (with senior team member) & 24 \\
\hline Regular review of caseload with senior team member or senior professional & 18 \\
\hline Individual supervision for at least 1 hour per month (with external supervisor) & 17 \\
\hline Peer consultation at least 2 hours per month (1-1) & 6 \\
\hline Consultation with psychiatrist (group) & 5 \\
\hline Other (please specify) & 3 \\
\hline Consultation with a psychiatrist (1 on 1) & 2 \\
\hline Other specified clinical supprvision/consultations or supprvisOry activities: \\
\hline
\end{tabular}

Other specified clinical supervision/consultations or supervisory activities:

- If external supervision occurs (and often does for more experienced team members) this is paid for by the individual.

- Clinical supervision mix dependent on staff's development stage. Have with varied level of experience (HEW 6,7,8 \& 9).

- Group clinical supervision from an external provider- 90 min per month.

Q61 Does the service offer supervised training placements for interns in psychology, social work, mental health etc?

Close to a third of managers $(\mathrm{N}=10)$ indicated that the service 'rarely' or 'never' provided supervised training placements for interns.

Table 67

\begin{tabular}{|c|c|}
\hline \multicolumn{2}{|c|}{ Supervised training placements for interns } \\
\hline Answer Choices & Number of Institutions \\
\hline Regularly & 20 \\
\hline Occasionally & 2 \\
\hline Rarely & 1 \\
\hline Never & 9 \\
\hline
\end{tabular}

The intern/s by profession/s were specified as: 
- Clinical Psych

- Psychologist - Counsellor

- Social Work, Counselling, Clinical Psychology

- Psychology, Counselling

- Psychology and Social Work

- Counsellors

- Clinical Psychology

- Counselling Psychology, Clinical Psychology, Occasionally 4+2 and 5+1 program placement students

- Master of Psychology (Clinical)

Q62 Does the service provide opportunities for students to undertake volunteer project work relevant to their degree and career within the service?

Only three (3) managers indicated that the service provides a formal student volunteer program.

Table 68

\begin{tabular}{|l|c|}
\hline \multicolumn{1}{|c|}{ Provide opportunities for students to undertake volunteer } \\
\multicolumn{1}{|c|}{ Answer Choices } & $\begin{array}{c}\text { Number of } \\
\text { Institutions }\end{array}$ \\
\hline Never & 17 \\
\hline $\begin{array}{l}\text { Occasionally - when a specific student with appropriate skills is } \\
\text { matched with a project }\end{array}$ & 7 \\
\hline Rarely & 6 \\
\hline Regularly - have a formal student volunteer program & 3 \\
\hline Please specify the student volunteer programs provided & 9 \\
\hline
\end{tabular}

Specified student volunteer programs provided:

- Exercise and mental health outcome. International student translation.

- Student wellbeing awareness team (SWAT) is supervised by one of our team.

- About to embark on taking a Public Health Student for a project based placement in semester 2.

- Student Minds - mental health awareness and reduction of stigma.

- Mental Health promotion events, supporting social groups targeting equity groups.

Q63 Does your counselling service offer any DEDICATED programs for students of specific backgrounds? LSES background, first in family to attend, or for students with disability?

Caution needs to be taken in understanding the responses to this question, as entry of more than one response may not have been possible given feedback received. Dedicated services for LGBTFIQ students was the most common response. 
Table 69

\begin{tabular}{|l|c|}
\multicolumn{1}{|c|}{ DEDICATED programs for students of specific backgrounds } \\
\hline \multicolumn{1}{|c|}{ Answer Choices } & $\begin{array}{c}\text { Number of } \\
\text { Institutions }\end{array}$ \\
\hline Students from Low Socio-Economic background & 1 \\
\hline Students First in Family to attend uni & 0 \\
\hline Students with a disability & 2 \\
\hline Indigenous students & 2 \\
\hline LGBTFIQ students & 6 \\
\hline Students whose first language is not English & 1 \\
\hline Students who are under 18 years of age & 0 \\
\hline Mature age entry students & 2 \\
\hline $\begin{array}{l}\text { Students studying in areas that are not traditional for their gender } \\
\text { identity }\end{array}$ & 0 \\
\hline Other (please specify) & 8 \\
\hline
\end{tabular}

Responses to other specified:

- No we don't offer any dedicated programs.

- Transition support for first year students with identified support needs other than disability

- Nil

- Not dedicated, but ad hoc-ly involved in indigenous; LGBTFIQ; Mature age

- Students considered at-risk (all of the above groups) have access to a targeted transition program.

- Nil

- All programs are open to all students

Q64 Please indicate which of the following apply to the service accommodation and infrastructure. Tick all that apply.

All managers indicated that 'Client records are adequately protected and secure'.

The majority of managers reported that:

- The service was 'accessible for students with disabilities'

- 'All client records are created and stored electronically'

- 'Furnishings satisfy comfort and OHS requirements'

- Counselling Service information is 'integrated within the institution's website'

- The service 'uses electronic case management software'

- The service was in a 'central location'

- The service 'has regular access to a suitable room for interactive workshops'

- 'Sound transfer levels adequate to ensure privacy during counselling sessions'

- The service has 'adequate rooms for counselling (size - minimum 13 metres square, light, warmth, cooling and ventilation)'

- 'Staff and students have access to information technology required for service delivery in a contemporary counselling service'

- 'Reception and waiting areas have adequate privacy' 


\begin{tabular}{|l|c|}
\hline \multicolumn{1}{|c|}{ Service accommodation and Infrastructure } & Number of Institutions \\
\hline Client records are adequately protected and secure & 31 \\
\hline Accessible for students with disabilities & 28 \\
\hline All client records are created and stored electronically & 28 \\
\hline Furnishings satisfy comfort and OHS requirements & 27 \\
\hline Counselling Service information integrated with institutions website & 27 \\
\hline The service uses electronic case management software & 25 \\
\hline Central location & 23 \\
\hline $\begin{array}{l}\text { The service has regular access to a suitable room for interactive } \\
\text { workshops }\end{array}$ & 23 \\
\hline $\begin{array}{l}\text { Sound transfer levels adequate to ensure privacy during counselling } \\
\text { sessions }\end{array}$ & 23 \\
\hline $\begin{array}{l}\text { Adequate rooms for counselling (size - minimum 13 metres square, } \\
\text { light, warmth, cooling and ventilation) }\end{array}$ & 21 \\
\hline $\begin{array}{l}\text { Staff and students have access to information technology required for } \\
\text { service delivery in a contemporary counselling service }\end{array}$ & 21 \\
\hline Reception and waiting areas have adequate privacy & 15 \\
\hline Discrete location & 11 \\
\hline A professional library is provided for staff & \\
\hline $\begin{array}{l}\text { Supervision of staff and interns can be conducted via direct observation } \\
\text { and/or audio-video recordings }\end{array}$ & 9 \\
\hline A self help library is provided for students & 6 \\
\hline Counselling Service information provided as a stand alone website & 5 \\
\hline Additional comments about existing service accommodation & 5 \\
\hline None of the above apply & 0 \\
\hline
\end{tabular}

Additional comments about existing service accommodation identified issues that managers felt were different to other similar services, or not ideal:

- Would like to have more information on a more modern website e.g. webinars/ workshop info - but we don't have time/technical knowledge; Existing rooms are approx. 12.3sqm; well lit, cooling/heating.

- Electronic/online client statistical database but counselling notes stored separately at this stage.

- Counsellors in our service are all based at different locations so some of the items selected apply to one or more of the counsellors, hence selection of discrete and central location.

- We are about to move to a less central location which is still not ideal but should be more discrete. We are currently in the same building as administration and our rooms have no soundproofing, inadequate ventilation (no windows in 3), and our desks are in a shared open plan area. We do not have case management software but place electronic notes on a secure server. Not ideal, but it is what we have at present. We have no group room and need to book a room somewhere on campus when needed.

- Based in prime student locations and co-located in a 'one stop shop' approach with other student services. 


\section{Service Accommodation and Infrastructure}

Additional comments about existing service accommodation

Counselling Service information provided as a stand alone website

A self help library is provided for students

Supervision of staff and interns can be conducted via direct observation and/or audio-video recordings

A professional library is provided for staff

Discrete location

Reception and waiting areas have adequate privacy

Staff and students have access to information technology required for service delivery in a. Adequate rooms for counselling (size - minimum 13 metres square, light, warmth, cooling and...

Sound transfer levels adequate to ensure privacy during counselling sessions

The service has regular access to a suitable room for interactive workshops

Central location

The service uses electronic case management software

Counselling Service information integrated with institutions website

Furnishings satisfy comfort and OHS requirements

All client records are created and stored electronically

Accessible for students with disabilities

Client records are adequately protected and secure

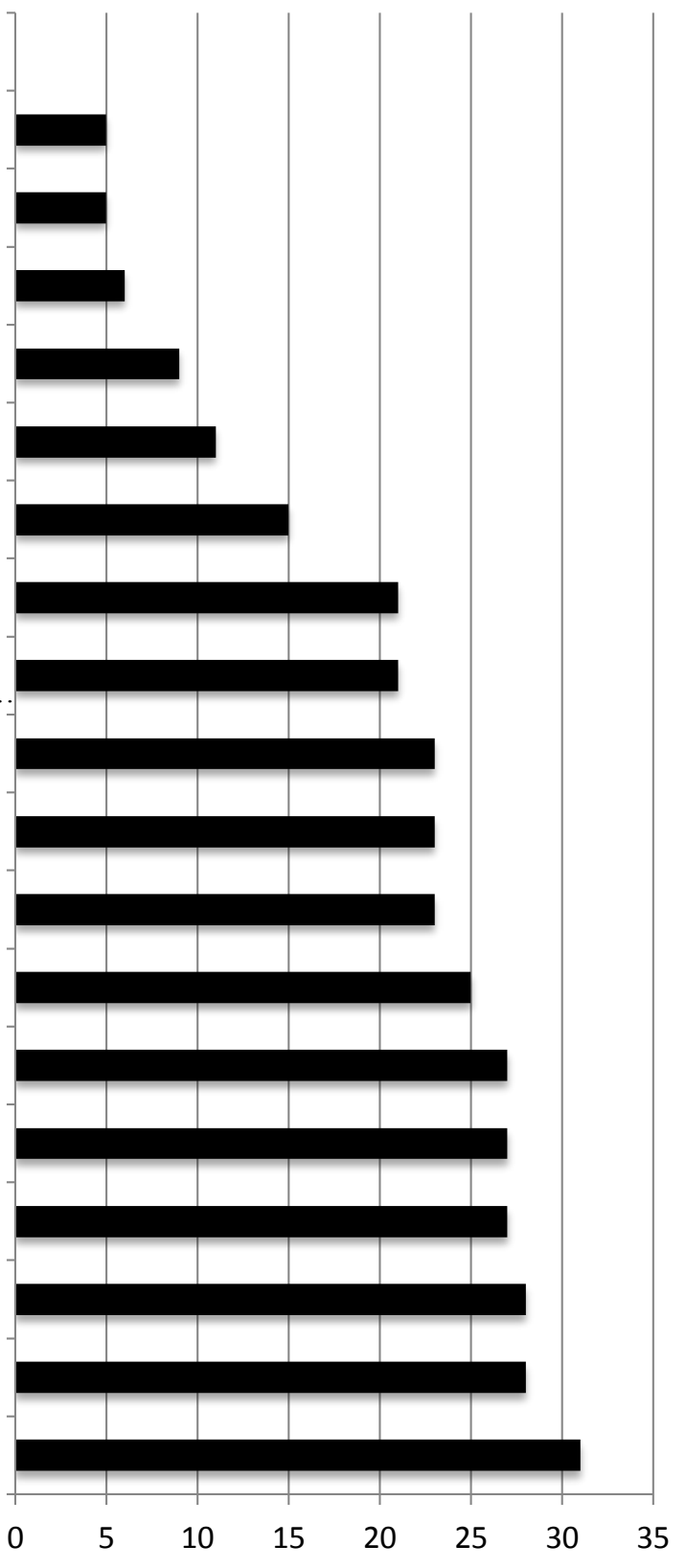

Figure 19

Q65 Which of the following activities has your service conducted in the last 2 years? Tick all that apply.

Twenty six (26) responses were received for 'Mental Health Awareness Programs/Workshops' and seven (7) reports were made for 'a formal for 'credit' course related to mental health and/or psychological literacy and/or psychological flexibility or resilience'. 


\begin{tabular}{|l|c|}
\hline \multicolumn{1}{|c|}{ Answer Choices } & $\begin{array}{c}\text { Number of } \\
\text { Institutions }\end{array}$ \\
\hline Mental Health Awareness Programs/Workshops & 26 \\
\hline $\begin{array}{l}\text { A formal for 'credit' course related to mental health and/or } \\
\text { psychological literacy and/or psychological flexibility or resilience }\end{array}$ & 7 \\
\hline Other systemic intervention program/s & 5 \\
\hline None of the above & 4 \\
\hline Anxiety Awareness Screening Day & 1 \\
\hline Depression Awareness Screening Day & 1 \\
\hline Other (please specify) & 8 \\
\hline
\end{tabular}

Other Specified responses:

- We run a variety of workshops \& groups related to mental health, but they're not a pure awareness program

- Annual wellbeing symposia National

- Accidental Counsellor

- Drug and Alcohol use screening tool; Meditation workshops

- An informal for 'no credit' course related to mental health and/or psychological literacy and/or psychological flexibility or resilience available on the learning platform (moodle/iLearn)

- Mental Health First Aid Training; R U OK day/Mental Health Week etc

- Unitopia; RU ok day

- RUOK Day; Mental health Week; Australian \& NZ University health and Wellbeing Day

Q66 Have you observed any 'emerging' areas of demand for service delivery in the last 12-18 months.

Only six (6) managers reported that they did not observe 'emerging' areas of demand for service delivery in the last 12-18 months. Conversely, twenty-six (26) managers reported that they had observed 'emerging' areas of demand for service delivery in the last 12-18 months.

Table 72

Observed 'emerging' areas of demand for service delivery in the last 12-18 months.

\begin{tabular}{|c|c|}
\hline Answer Choices & Number of Institutions \\
\hline No & 6 \\
\hline Yes & 26 \\
\hline
\end{tabular}

Specified observations:

- More frequent presentations of risk, trauma and complexity.

- HUGE DEMAND TO DEVELOP MINDFULNESS SKILLS.

- Continued increase in presentations from students with complex needs and underlying personality issues.

- More Mental Health related issues \& difficulty of engaging Crisis Mental Health teams.

- Govt is currently running an Enquiry into the adequacy of Mental Health \& Addictions. We are providing a submission, as is the University.

- Transgender students services required for hormone treatment pathway 
- Difficult Behaviours; Sexual Misconduct.

Q67 If you answered 'Yes' to the question above: What action have you taken so far to respond to these emerging areas?

Managers reported many actions taken in response to the 'emerging issues':

- Attempted to provide a service. Communicated / advocated to managers about need for more resources.

- Online programs, extensive mindfulness workshops.

- Increased service hours dedicated to urgent response by senior counsellors. Increased training opportunities for counsellors in interventions to suit BPD and ACE history.

Referral options explored.

- Endeavouring to target groups \& workshops to either manage or help prevent onset. However, at times, we have limited attendees!

Limited to no success in engaging Mental Health Services.

- Entered into partnership with local hospital to run a pilot trans-affirmative care pilot project.

- Specialised training and consultation.

Formation of a specific sexual misconduct support unit.

- Developed a Mental Health Basic Program run 2-3 times per semester.

ALERT Training.

- Used recruitment to employ appropriate clinicians.

Set up a Support Group.

- Updating of staff response protocols, policies, awareness raising and more training for counsellors and other responding staff.

- Training and specialised supervision provided for Counselling Team on working with clients who have experienced trauma, specifically domestic violence and/or sexual violence. Review of existing policy and development on new Student Critical Incident Policy. Advocacy officer appointed at the University.

Advocating for a Safer Community Unit to be established to manage behavioural misconduct complaints that cause psychological harm/or involve.

bullying/harassment/assault.

- Specialised training for counsellors.

Developed facts sheet specific to university.

Participant in advisory group.

Workshops for student residents.

- Some initial service delivery - telephone \&/or Zoom/Skype.

Currently a project is in place to address this need and increase service and practitioner capacity in this space.

- Involved in their orientation days and short workshops ad hoc.

No funds available for specific programs or targeted staffing.

- We have increased our staffing specifically in Duty / Triage response - so we can manage the flow of students into the service and the interactions with and referrals to other supports. 
- Education ie MHFA and MHFA for the Suicidal Person.

Increased liaison with community services, in particular those with expertise in PTSD MOU with Headspace at bigger campus to support referral of students.

- Business case to ensure Counselling levels are sustained.

- More clinics.

- Establish an international student program.

- Delivered some training to groups of staff (but still too ad-hoc).

Developed some resources for staff.

Refined a case management plan template.

Working alongside the Respect. Now. Always project officer re: training options and resource development.

Input given to relevant Committees responding to student progression matters.

- Developed student workshops, presented training to other student services staff, reviewed online information including access to self help tools.

- Attempting to build closer connections with Graduate Research Office.

- 1) Restructured our service delivery model to increase access to on the day appointments (these are only made available on the day or for the next day)

2) Employed a Mandarin speaking psychologist

3) Employed more clinical psychologists.

- Put a case to the service Director for increased FTE (not yet responded to)

Written of concern for the welfare of staff having to manage these clients with minimal safety support in place (no medical clinical on site, no psychiatrist on site) and seeing distressed clients in between booked appointments and during lunch breaks.

- Increased marketing to make students and staff aware we are not a crisis service.

- Social anxiety - blogs and online resources.

- Frameworks created.

Q68 Does your service provide counselling and psychological services beyond usual business hours available Mon-Fri (9 -5)?

Managers most frequently $(\mathrm{N}=9)$ reported providing after-hours counselling and psychological services 'by arrangement'.

Table 73

Provide counselling and psychological services beyond usual business hours available Mon-Fri (9 -5)

\begin{tabular}{|l|c|}
\hline \multicolumn{1}{|c|}{ Answer Choices } & Number of Institutions \\
\hline $\begin{array}{l}\text { Yes - Occasionally by arrangement before 9am after 5pm } \\
\text { on Mon-Fri only. }\end{array}$ & 11 \\
\hline $\begin{array}{l}\text { Yes - Face-to-Face Services are provided 9-5 Mon-Fri and a } \\
\text { dedicated after hours telephone counselling service provides } \\
\text { extended hours coverage outside of business hours. }\end{array}$ & 3 \\
\hline Never & 9 \\
\hline $\begin{array}{l}\text { Yes - Routinely during the whole of the year the service is } \\
\text { open for extended hours before 9 am and after 5 pm Mon-Fri } \\
\text { only. }\end{array}$ & 1 \\
\hline
\end{tabular}

Journal of the Australian and New Zealand Student Services Association: 


\begin{tabular}{|l|c|}
\hline \multicolumn{1}{|c|}{$\begin{array}{c}\text { Provide counselling and psychological services beyond usual business hours available } \\
\text { Mon-Fri (9 -5) }\end{array}$} \\
\hline \multicolumn{1}{|c|}{ Answer Choices } & Number of Institutions \\
\hline $\begin{array}{l}\text { Yes - The service provides a 24/7 service including extended } \\
\text { hours Mon-Fri and a dedicated after-hours telephone } \\
\text { counselling service. }\end{array}$ & 0 \\
\hline $\begin{array}{l}\text { Yes - During peak demand periods the service is open for } \\
\text { extended hours before 9 am and after 5 pm Mon-Fri only }\end{array}$ & 0 \\
\hline $\begin{array}{l}\text { Yes - Routinely during teaching weeks the service is open for } \\
\text { extended hours before 9 am and after 5 pm Mon-Fri only. }\end{array}$ & 0 \\
\hline $\begin{array}{l}\text { Yes - Routinely the service is open for extended hours Mon- } \\
\text { Fri and also some hours on Sat and Sun. }\end{array}$ & 0 \\
\hline
\end{tabular}

Other specified responses show that services do provide some flexibility for students beyond 9-5 service delivery hours:

- 8-5 at St Lucia campus. 10-6 at a Herston campus. 8-4 at Gatton Campus.

- We are open one late night until 7 pm

- We open on Saturdays over the exam periods

- Emergencies; critical incident management and hospitalizations

- 8-6 throughout the year, Mon-Fri

- In response to crisis or critical incident, for one or two week duration. Service has utilised University EAP provider for afterhours and weekend support.

- Between Feb and Nov the service is open 9am till 6pm on Tuesday, Wednesday and Thursday. $9 \mathrm{am}-5 \mathrm{pm}$ for all the rest of the time.

- Extended hours from 5:00 - 7:00 pm Tuesday and Wednesdays.

Q69 Does your service provide counselling and psychological services on Saturday or Sunday?

Provision of counselling and psychological services on Saturday or Sunday was not offered by more than one (1) service only an occasional basis. Twenty-five (25) managers recorded that services on Saturday or Sunday were not offered.

Table 74

\begin{tabular}{|l|c|}
\hline \multicolumn{2}{|c|}{ Counselling and psychological services on Saturday or Sunday } \\
\hline \multicolumn{1}{|c|}{ Answer Choices } & $\begin{array}{c}\text { Number of } \\
\text { Institutions }\end{array}$ \\
\hline Never & 25 \\
\hline Yes - Occasionally & 1 \\
\hline Yes - Routinely during teaching weeks only & 0 \\
\hline Yes - Routinely during the whole of the academic year & 0 \\
\hline
\end{tabular}

Other responses

- Unless called out by Accommodation service in emergency.

- Saturday clinic during exam times only

- During exam periods we are open on Saturdays

- In response to a Student Critical Incident part of the management plan was to check in with the at risk student over the weekend.

- After hours phone/text crisis support 
- Usually only in response to critical incidents.

Q70 Which of the following does your service provide in an online/website environment for student and university community access? Tick all that apply.

All managers reported online/website environment service provision. The majority $(\mathrm{N}=32)$ identified use of a Static website with service information, tip sheets and brochures $(\mathrm{N}=24)$ and self-paced learning materials $(\mathrm{N}=12)$. Only nine (9) managers indicated the provision of online counselling.

Table 75

\begin{tabular}{|l|c|}
\hline \multicolumn{2}{|c|}{ Service online/website environment } \\
\hline \multicolumn{1}{|c|}{ Answer Choices } & Number of Institutions \\
\hline Static website with service information & 32 \\
\hline Tip sheets and brochures & 24 \\
\hline Self paced learning materials & 12 \\
\hline Online counselling & 9 \\
\hline 'Q and A' posting/response & 4 \\
\hline Online chat/forum options & 2 \\
\hline None of the above & 0 \\
\hline
\end{tabular}

Other specified responses:

- Skype counselling. Trying to post webinars online, but have limited skill \& technical support to do so.

- Online counselling via video conferencing only, not email.

- Online booking facilities

- Have an online treatment program + referral is completed via an online screening form.

- We have an online forum at discrete times of the year in a module that includes other services' forums as well (eg Careers). We have some self-paced learning materials within the module.

- Student Health and Wellbeing Blog

Q71 Commuter students who travel considerable distances to attend campus can experience a reduced opportunity to utilise student services and may have difficulty attending for needed counselling. Does your service address this need for service provision in any particular way?

Only six (6) managers indicated that commuter students were not catered for by the service they managed. A number of mangers $(\mathrm{N}=14)$ indicated that commuter students were catered for routinely and eleven (11) identified that commuter students were offered services occasionally following specific request. Telephone and Zoom or Skype services were identified in 'other' responses.

Table 76

\begin{tabular}{|c|c|}
\hline \multicolumn{2}{|c|}{ Services that cater for commuter students } \\
\hline Answer Choices & $\begin{array}{l}\text { Number of } \\
\text { Institutions }\end{array}$ \\
\hline Yes - routinely during the whole of the academic year & 14 \\
\hline Occasionally on specific request & 11 \\
\hline Not at all & 6 \\
\hline Yes - during teaching weeks only & 1 \\
\hline Not applicable - provide distance education only & 0 \\
\hline
\end{tabular}

Other specified responses: 
- Counselling service is provided over the phone.

- Set appointment later in the day, remote counselling (phone, etc)

- Telephone appointment

- We offer Skype appointments

- Telephone and online video counselling available, appointments after hours be arrangement, online self-help resources.

- Zoom (Virtual Face to Face) and phone sessions are offered to all students

- Online counselling via Skype, phone or email

After hours Skype drop in

Occasional webinars

- Appointments outside business hours

- We offer telephone counselling sessions + an online treatment program which is available $24 / 7$ over a 5 week period with email or telephone consults with a psychologist.

- Phone consultations are offered as well as regular email check ins

- Will conduct phone and Skype-based counselling during normal business hours

- Offer phone counselling

- We provide phone and Skype counselling for students living away from campus, attending placements, etc.

- Can access counselling service via phone/online or in person by arrangement after or before hours. Usually in response to a specific need or critical incident. Counsellors may attend an alternative site to offer services by arrangement.

- We can use Zoom for appointments if it is deemed suitable

- Outreach to other campuses

Skype and Phone counselling

Regional and remote contract counsellors

- Extended hours, after hours crisis support, phone and online counselling

- Face to face counselling provided through the EAP provider for students on placement in regional and remote locations, on a case-by-case basis.

Q72 Part time students frequently attend class outside of business hours. Does your service provide any services that specifically address the needs of these part time students?

Approximately $43 \%$ of managers indicated that services suited to part-time student needs are provided. The remaining $57 \%$ of managers did not offer services that specifically addressed the needs of part-time students.

Table 77

\begin{tabular}{|c|c|}
\hline \multicolumn{2}{|c|}{ Services offered that suit part-time students } \\
\hline Answer Choices & Number of Institutions \\
\hline No & 17 \\
\hline Yes & 13 \\
\hline
\end{tabular}

Other specified responses:

- We are open till 6pm 4 days a week 
- $\quad$ Skype - a/a

- After Hours counselling telephone services is available out of hours.

- After Hours appointments by arrangement, telephone and video counselling available, online self-help resources.

- Zoom (Virtual Face to Face) and phone sessions are offered to all students.

- Online counselling via Skype, phone or email

After hours Skype drop in

Occasional webinars

- Appointments outside business hours

- We offer telephone counselling sessions + an online treatment program which is available $24 / 7$ over a 5 week period with email or telephone consults with a psychologist.

- Phone consultations are offered as well as regular email check ins

- Will conduct phone and Skype-based counselling during normal business hours

- After hours crisis line

- Phone after hours by arrangement or asynchronous email therapy http://www.utas.edu.au/students/shw/counselling/online

- We have 5pm appointments three days per week

- Extended hours, after hours crisis support, phone and online counselling

- $24 / 7$ phone counselling

Online counselling

Q73 Which types of counselling appointments does your counselling service provide? Tick any that apply.

Twenty-five (25) responses indentified 'urgent appointment options available each day' and twenty-four (24) responses indentified 'telephone counselling appointments'. Twenty (20) respondents 'limited ongoing counselling appointments by service policy' and 'flexible modification to session limits for counselling appointments was offered 'as needed to address the mental health needs of the student' by twenty (20) managers. Twelve (12) managers indicated that 'first appointments (60 minutes) could be pre-booked in advance '.

Table 78

\begin{tabular}{|l|c|}
\hline \multicolumn{1}{|c|}{ Types of counselling appointments provided } \\
\multicolumn{1}{|c|}{ Answer Choices } & $\begin{array}{c}\text { Number of } \\
\text { Institutions }\end{array}$ \\
\hline Urgent appointment options available each day & 25 \\
\hline Telephone counselling appointments & 24 \\
\hline Ongoing counselling appointments limited by service policy & 20 \\
\hline $\begin{array}{l}\text { Flexible modification to session limits for counselling appointments as needed } \\
\text { to address the mental health needs of the student }\end{array}$ & 20 \\
\hline First appointments pre-booked in advance (60 minutes) & 12 \\
\hline Drop-in /on the day appointments (30 minutes) only bookable on the day & 11 \\
\hline Email counselling & 11 \\
\hline $\begin{array}{l}\text { Telephone and face-face triage system for screening severity of presentation for } \\
\text { clients prior to first assessment }\end{array}$ & 9 \\
\hline Walk-in and wait for appointment option & 8 \\
\hline
\end{tabular}




\begin{tabular}{|l|c|}
\hline \multicolumn{1}{|c|}{ Types of counselling appointments provided } \\
\multicolumn{1}{|c|}{ Answer Choices } & $\begin{array}{c}\text { Number of } \\
\text { Institutions }\end{array}$ \\
\hline \multicolumn{1}{|c|}{} & 7 \\
\hline Drop-in appointments (60 minutes) only bookable on the day & 7 \\
\hline $\begin{array}{l}\text { Face-face triage system for screening severity of presentation for clients prior } \\
\text { to first assessment }\end{array}$ & 7 \\
\hline Online counselling (asynchronistic) & 5 \\
\hline First appointments pre-booked in advance (30 minutes) & 4 \\
\hline $\begin{array}{l}\text { Telephone triage system for screening severity of presentation for clients prior } \\
\text { to first assessment }\end{array}$ & 4 \\
\hline Online chat in real time (synchronistic) with a counsellor & 3 \\
\hline Unlimited ongoing counselling appointments & 1 \\
\hline $\begin{array}{l}\text { Focused session appointments (60 -90 minutes) with follow up limited to } \\
\text { phone, email or an additional 30 minute appointment }\end{array}$ & 11 \\
\hline Other (please specify) & \\
\hline
\end{tabular}

Other specified responses:

- $\mathrm{V} / \mathrm{C}$ counselling

- Email Triage questionnaire for screening risk prior to 1st assessment. 30 minute appts for Impaired Performance/Aegrotats leading up to/during exam periods

- Online video conferencing counselling appointments

- ZOOM (Virtual face to face)

- Online treatment program; 3 free for all students then (up to 10 more on MHCP)

- Skype-based counselling

- Triage of acute students by medical staff and then managed until counselling apt available

- Use WebEx to offer students counselling via the internet when attendance at a campus is not possible

- We have urgent appointments only for the counsellor within our team who only sees students within the residential system. We set aside 2 hours admin at the end of each day that can be used for emergencies as needed. We do not offer "email" counselling but will respond to short information requests via the secure counselling queue in AskUNE. We offer asynchronistic chat via online forums from time to time.

- Initial Consultations for new clients are 45 minutes (15 minutes pre-screening, 30 minutes with Counsellor). These include some triage and immediate service delivery if applicable, and they are booked on the day or can book for the next day. These can be followed by up to 6 Return appointments of 1 hour each.

- Skype counselling 


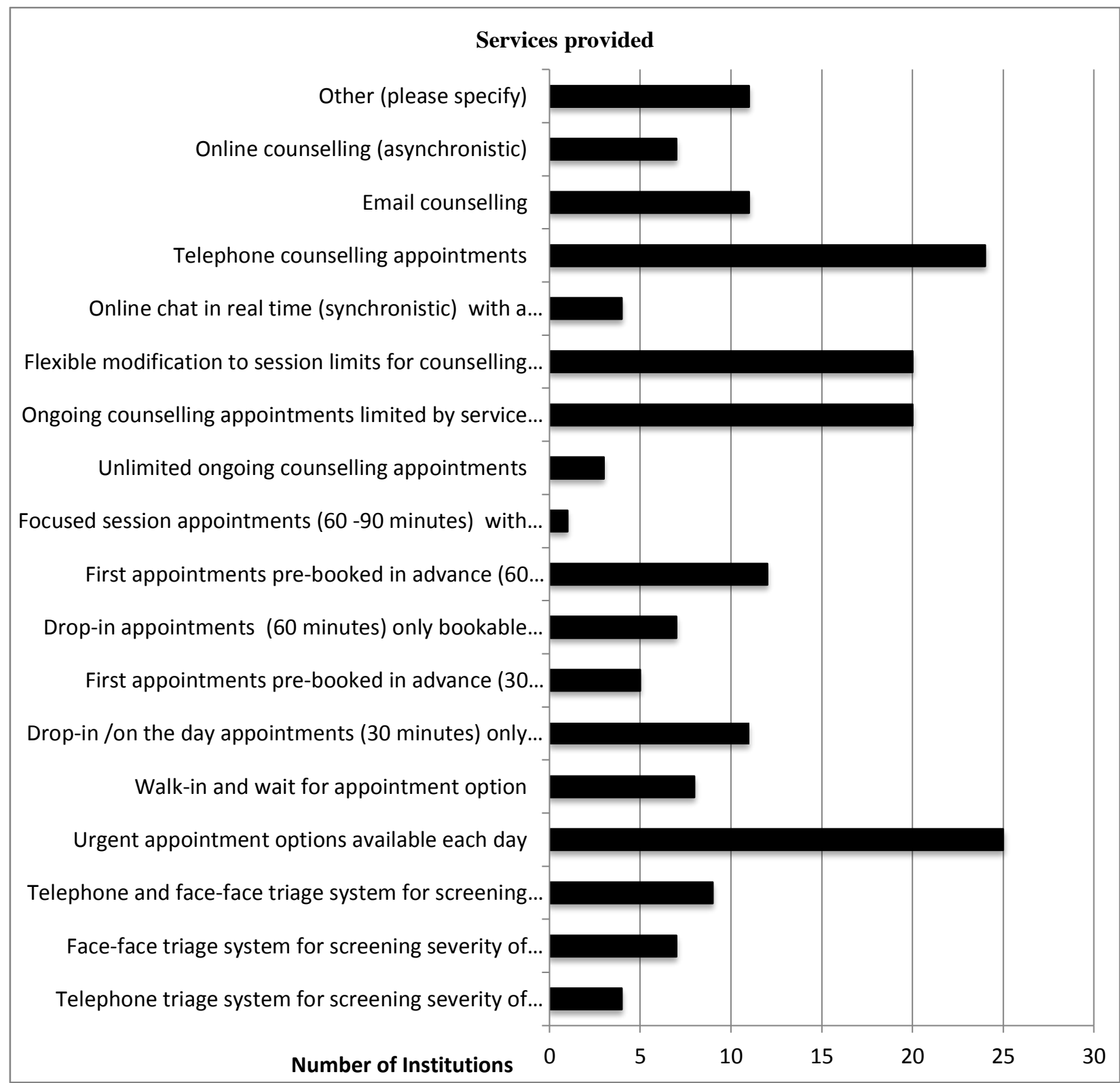

Figure 20

Q74 What is the advertised upper limit of counselling appointments that a student/client can access in each year in your service?

Range for 'session limits advertised for counselling' was 'no limit' to 13 sessions/appointments. Ten (10) managers identified no advertised limit to counselling appointments. Seven (7) managers identified six (6) appointments as the advertised limit. 
Table 79

\begin{tabular}{|c|c|}
\hline \multicolumn{2}{|c|}{ Advertised limit of counselling appointments } \\
\hline Number of appointments & Number of Institutions \\
\hline No limit identified & 10 \\
\hline 6 appointments & 7 \\
\hline 8 appointments & 1 \\
\hline 10 appointments & 1 \\
\hline 12 appointments & 1 \\
\hline 13 appointments & 7 \\
\hline Other & \\
\hline
\end{tabular}

Specific Responses:

- 6 per semester

- Has been 10, but I would prefer that we removed that advertising \& it was determined by practitioner/client need; following consult with Manager to go over 10. Manage that limit by encouraging students to participate in groups.

Usual averages are 3-4 per person.

- No limit advertised, but in practice 6-10.

- Extended to 9 if pre-approved by manager - on a case by case basis

- 5-6 but on an individual basis. International can see private unlimited on insurance

- Each client usually has one initial consultation and up to 6 returns. If more than 3 initial consultations are initiated in one year then the Senior Counsellor would follow up with the client.

- 4 sessions per study period - advertised as a brief intervention service

Q75 The 2015 -2016 AUCCCD survey reports the mean number of counselling appointments utilised per student attending the university or college counselling service ranged from 4.64-6.07. In 2017, what was the mean number of appointments utilised by the clients within the counselling service that you manage?

The range for the average number of sessions utilised by clients was 1.5 to $>17$. The most common 'average number of sessions' was 2.5 identified by ten (10) managers.

Table 80

\begin{tabular}{|c|c|}
\hline \multicolumn{2}{|c|}{ Mean number of appointments utilised by the clients } \\
\hline Average number of Sessions & 1 \\
\hline 1.5 & 1 \\
\hline 2 & 10 \\
\hline 2.5 & 6 \\
\hline 3 & 6 \\
\hline 3.5 & 1 \\
\hline 4 & 1 \\
\hline 4.5 & 3 \\
\hline 5 & 1 \\
\hline 5.5 & 1 \\
\hline 8 & 1 \\
\hline$>17$ & \\
\hline
\end{tabular}




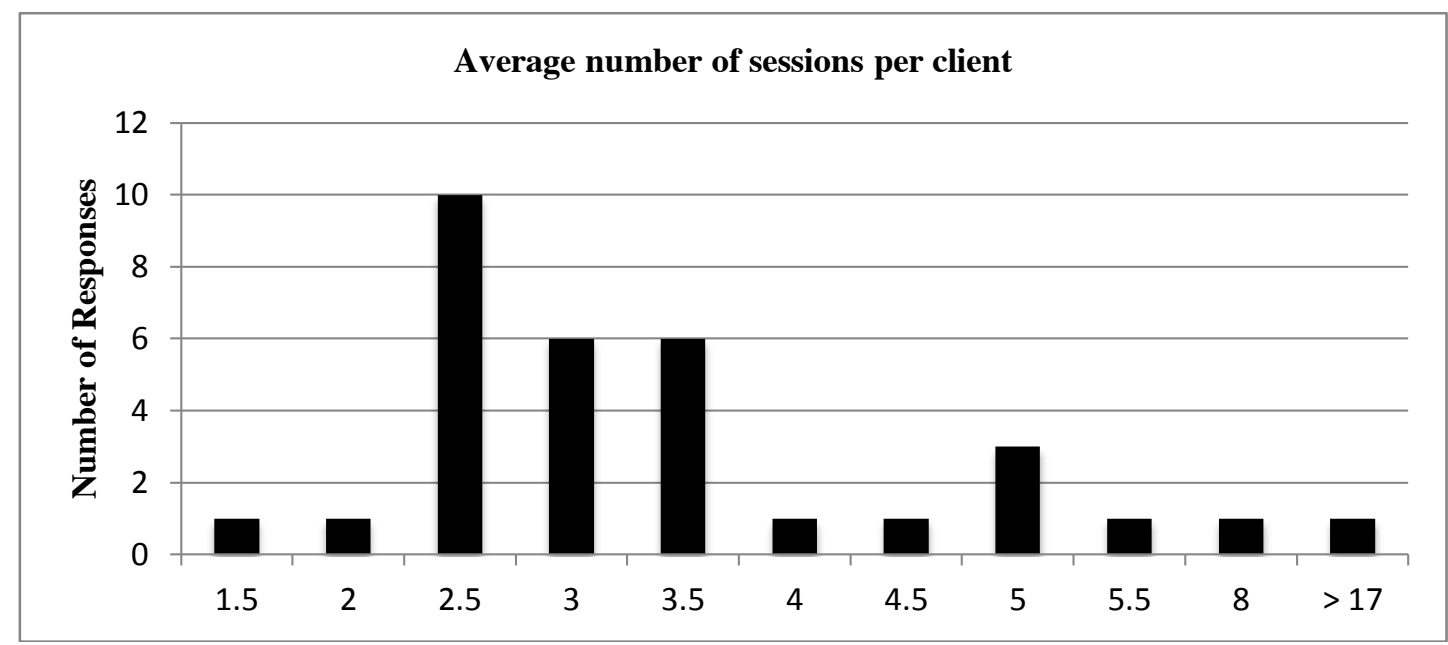

Figure 21

Q76 Does the service utilise a waiting list or unmet demand list?

Almost forty-one (41) percent of managers indicated that they utilised a 'wait list or unmet demand list'. The remaining fifty-nine (59) percent did not utilise a 'wait list or unmet demand list'.

\begin{tabular}{|c|c|}
\hline \multicolumn{2}{|c|}{ Use wait list/unmet demand list } \\
\hline Answer Choices & Number of Institutions \\
\hline No & 19 \\
\hline Yes & 13 \\
\hline
\end{tabular}

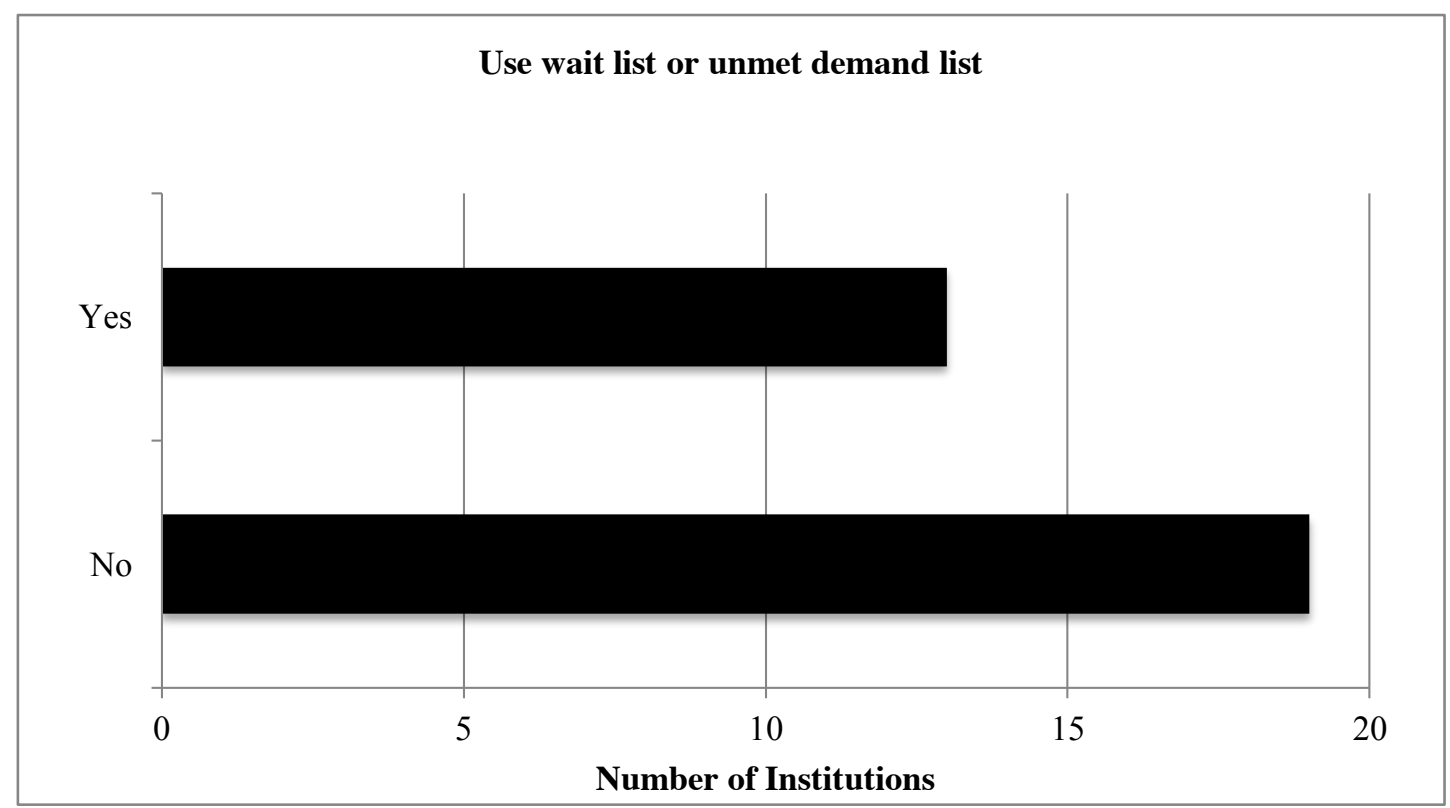

Figure 22

Q77 Are students registered on the wait list or unmet demand list offered appointments as available due to cancellations?

All managers utilising a waitlist/unmet demand list offered appointments released by cancellations. 
Table 82

Students registered on the wait list or unmet demand list offered appointments as available due to cancellations

\begin{tabular}{|c|c|}
\hline Answer Choices & Number of Institutions \\
\hline No & 0 \\
\hline Yes & 12 \\
\hline Not applicable - wait list/unmet demand list not utilised & 1 \\
\hline
\end{tabular}

Q78 Does the service use a screening tool to assess severity of psychological distress prior to placement on wait list/unmet demand list?

Only two (2) of twelve (12) managers used a screening tool to assess severity of psychological distress prior to placement on a wait list/unmet demand list.

Table 83

\begin{tabular}{|c|c|}
\hline \multicolumn{2}{|c|}{$\begin{array}{c}\text { Use of screening tool to assess severity of psychological distress prior to } \\
\text { placement on wait list/unmet demand list }\end{array}$} \\
\hline \multicolumn{1}{|c|}{ Answer Choices } & Number of Institutions \\
\hline No & 10 \\
\hline Yes & 2 \\
\hline $\begin{array}{l}\text { Not applicable - wait list/unmet demand } \\
\text { list not utilised }\end{array}$ & 1 \\
\hline
\end{tabular}

Specific Response:

- Only for our drop-in appointments

Q79 Does your service utilise a psychological distress screening tool such as the Kessler 10.

Four (4) of twelve (12) managers identified the use of a psychological distress screening tool within the service.

Table 84

\begin{tabular}{|c|c|}
\hline \multicolumn{2}{|c|}{ Use a psychological distress screening tool } \\
\hline Answer Choices & Number of Institutions \\
\hline Yes & 4 \\
\hline No & 8 \\
\hline
\end{tabular}

Specific Responses:

- Celesthealth

- Kessler 10

- K10 / DASS at discretion of practitioner

- DSM-V screening tool + extra questions

- We do not use these as a screening tool prior to first appt. but use them within appointments as needed. - eg K-10, DASS, BDI

- DASS 21 but only as part of the initial consultation

Q80 If a screening tool is used does the service have a policy/procedure that guides service delivery when the screening tool scores indicate acute psychological distress and an immediate appointment is not available? e.g. the student is to be followed up with a phone consultation within $24-48$ hours (i.e. within 2 working days)? 
Table 85

\begin{tabular}{|c|c|}
\hline $\begin{array}{c}\text { Policy/procedure that guides service delivery when the screening tool scores indicate } \\
\text { acute psychological distress and an immediate appointment is not available }\end{array}$ \\
\hline Answer Choices & Number of Institutions \\
\hline No & 2 \\
\hline Yes & 5 \\
\hline Not applicable - do not use screening tools & 6 \\
\hline
\end{tabular}

Specific Responses:

- Clients who are deemed as suicidal will be seen in an emergency appointment

- We have developed a triage form that all students complete at first contact or if in crisis

- K10 / DASS used at the start of first appointment with counsellor not as a screening tool to facilitate appointment.

- If the K-10 or other tool (eg DASS), or the clinical interview itself indicate high distress, and if there are also indicators of risk of harm to self or other, then another appt. is offered to the student asap after the appt. in which the distress has been flagged.

Q81 Does the service request that each student complete a substantial screening tool prior to or at the first scheduled appointment?

Eleven (11) of thirteen (13) managers ( $>84 \%$ ) indicated that a screening tool was not used prior to or at the first scheduled appointment.

Table 86

\begin{tabular}{|c|c|}
\hline \multicolumn{2}{|c|}{ Use of screening tool prior to or at the first scheduled appointment } \\
\hline Answer Choices & Number of Institutions \\
\hline No & 11 \\
\hline Yes & 2 \\
\hline
\end{tabular}

Specific Responses:

- https://secure.mq.edu.au/wellbeing/view.php?id=18298

- DASS 21 and also our own Health and Wellbeing Survey

Q82 Does your service offer a telephone or VOIP (e.g. SKYPE or VOOM) counselling service?

Managers indicated that use of telephone and VOIP counselling was offered by the service. Only seven (7) managers indicated that neither was offered.

Table 87

\begin{tabular}{|c|c|}
\hline \multicolumn{2}{|c|}{ Offer a telephone or VOIP counselling service? } \\
\hline Answer Choices & Number of Institutions \\
\hline No & 7 \\
\hline Yes - telephone counselling service & 13 \\
\hline Yes - a VOIP counselling service & 11 \\
\hline
\end{tabular}

Q83 Please briefly explain the options for after hours mental health services available to the students of your institution. Include external community services to which students are referred by your service website or phone message.

Managers identified many options for students to use when needing after hours support for mental health.

Responses provided: 
- CDU has an external Support Line for after-hours telephone support to students (provided by Life Line)

- MH crisis services. Phone help lines. Residential students have on-call student RA. Online services eg. Beyond Blue.

- 24-hour crisis services Agency Contact:

Life threatening emergencies (police/fire/ambulance) 000

Police (non-emergency) 131444

Sexual Assault Resource Centre +61893401828

Alcohol and Drug Information Service +61 894425000

1800198024 (Free call for regional WA)

Healthdirect 1800022222

- $\quad$ NSW Health Mental Health Line 1800011511

Life Line 131114

Suicide Call Back Service 1300659467

Kids Help Line 1800551800

The Salvation Army Hope Line 1300467354

Mensline Australia 1300789978

Veterans Line 1800011046

Beyond-blue 1300224636

- Students are referred to Lifeline; Crisis Care; Youthline; eheadspace; ED

- Public Health Crisis Mental Health teams or local Accident \& Medical Centres.

We include Mental Health Foundation/Lifeline/Youthline/ Anxiety NZ phone numbers on triage questionnaires.

- Crisis service at local hospital

Several 24/7 phone counselling services nationally provided

- After hours phone message directs to lifeline or 000

- After Hours telephone counselling service provided by university.

After Hours telephone line provided externally e.g. Life Line, Suicide Call back Service Mental Health Triage

- There is an answer phone that is answered by a nurse after hours and all urgent mental health issues are referred to Community Mental Health Services.

- Some after hours counselling appointments available. Also referred to the ACT Health Crisis Assessment and Treatment Service, Lifeline and Beyond Blue telephone helpline.

- In case of an emergency please contact 000 or go to your nearest emergency department. For 24hr crisis support please phone Lifeline on 131114.

To access other support services phone Beyond Blue on 1300224636 or access the Beyond Blue website https://www.beyondblue.org.au/get-support/get-immediate-support

- After-hours Crisis Support line between 5pm to 9am weekdays, and 24 hours over the weekends and public holidays. Phone or text chat options available.

Skype Drop in (no appointment) 8pm - 9pm twice a week.

Website directs to external services such as Lifeline, MH Advice Line and 000

- After hours student help line (BUPA)

Lifeline

Emergency Services/Hospital 
- Mental Health Triage- State Government service

Lifeline

Hospitals

- Use of community services such as Lifeline, Beyond Blue, Suicide Call back service, Respect, etc. The University also has an after hours student hotline to triage low-mid level inquiries and refer to services in community if needed.

- 24/7 care line (managed by external provider with Uni staff on call for consultation).

- Lifeline, beyond blue, mental health line + emergency services + sexual assault counselling services are used.

- If you are in distress or require immediate assistance, please call the ACT Mental Health Crisis Assessment and Treatment Team (CATT) on 62051065 or 1800629354 or contact Lifeline on 1311 14. If it is an emergency, please call 000.

- List of local emergency psychiatric services and online services.

- Emergency dept or mental health acute line

- After Hours crisis line- phone or SMS support

- Lifeline or 000

- We direct students to local, state and national mental health support services, numbers and web sites. We direct students to the 24/7 mental health support line in their state as needed. We do this via our webpage, as a footer in our emails, and via our urgent help webpage, and a Safety App for students. For students in our area, we direct them to the local hospital and community services.

- Community mental health, telephone helplines

- Lifeline

Mental Health Hot line (Government)

Local Hospital and CAT support (where applicable)

- No after hours counselling provided. Students referred to local hospitals, lifeline, suicide call back line etc.

- The only after hours services available are the local hospital or crisis lines.

- If your situation is urgent, please consider the Mental Health Triage 24 hour service on 13 14 65, Lifeline on 131114 or the Beyondblue helpline on 1300224636.

Both Lifeline and Beyondblue have online text chat support options as well: https://www.lifeline.org.au/

https://www.beyondblue.org.au/get-support/get-immediate-support

- After hours telephone counselling provided by the institute

Multiple options available privately and publicly throughout Melbourne city and metropolitan area

- After hours crisis responses services currently provided by University Crisis line. Also emergency mental health services utilised.

- $24 / 7$ phone counselling provided by external provider of the university's EAP.

Q84 Does the counselling service provide counselling options that are routinely available 24/7?

Only nine (9) counselling services provide counselling options routinely available 24/7. 


\begin{tabular}{|c|c|}
\hline \multicolumn{2}{|c|}{ Counselling available 24/7 } \\
\hline Answer Choices & Number of Institutions \\
\hline No & 22 \\
\hline Yes & 9 \\
\hline
\end{tabular}

Some managers elaborated on what services are provided $24 / 7$ and who provides these $24 / 7$ service/s:

- The Crisis Support Line provides those services.

- Only external community NGOs \& mental health services - see previous answer

- Yes - telephone counselling is provided $24 / 7$. 9-5pm provided by the counsellors at the service.

- Afterhours provide by third party provider (Converge)

- Newcastle provides an afterhours crisis support phone line between $5 \mathrm{pm}$ to 9am weekdays, and 24 hours over the weekends and public holidays.

Student Residents have After-hours Duty Officers however these are somewhat more similar to security with focus on support rather than counselling or welfare.

- After hours student help line (BUPA). Lifeline. Emergency Services/Hospital

- 5 week online treatment program (uniWellbeing) delivered by CAPS staff in partnership with the eCentreClinic

- National

Ambulance: 000

Police: 000

Lifeline (24 hrs): 131114

Suicide Prevention \& Crisis Intervention (24hrs) 1300363622

Kids Help Line (under 25yrs) on 1800551800

Suicide Callback Service 1300659467

Grief Support (24 hr assistance) on (02) 94896644 or 1800642066

Domestic Violence Crisis Line (emergency accommodation, crisis counselling, \& referral) on 1800656463

1800-RESPECT : 1800737732

Mentalhealthonline - Lists national and state mental health crisis service details.

- NSW

NSW Rape Crisis Centre: 1800424017

NSW Mental Health Line: $24 \mathrm{hr}$ service (to connect you with the right care) 1800011511

Alcohol and Drug Information Service (24 hour assistance) on (02) 93618000

- Armidale

Call 000 for Emergencies

Armidale Police Station (02) 67710699

Armidale Sexual Assault Service (02) 67769655 (BH) (02) 67769500 (AH)

UNE Safety \& Security (24hrs) (02) 67732099

Armidale Hospital 24 hr Accident \& Emergency (02) 67769622

- Community mental health help lines

- Telephone after hours

- As previous - University Crisis line answering UniSA out of hours crisis line phone and text 
- See previous answer

Q85 Which of the following strategies does your service employ to minimise "no shows" for scheduled appointments? Tick all that apply.

Most managers $(\mathrm{N}=24)$ reported using SMS to confirm appointments one or two days prior. Email reminders were also utilised.

Table 89

\begin{tabular}{|l|c|}
\hline \multicolumn{2}{|c|}{ Strategies to minimise "no shows" for scheduled appointments } \\
\hline \multicolumn{1}{|c|}{ Answer Choices } & $\begin{array}{c}\text { Number of } \\
\text { Institutions }\end{array}$ \\
\hline SMS prior one or two days before to confirm appointment & 24 \\
\hline $\begin{array}{l}\text { Apply an administrative fee or charge/fine for non } \\
\text { attendance }\end{array}$ & 6 \\
\hline Phone call the day before to confirm appointment & 4 \\
\hline None of the above & 5 \\
\hline Other (please specify) & 5 \\
\hline
\end{tabular}

Other specified responses:

- Deal with on a case-by-case basis for students who have a history of no shows (i.e. limiting future access to the service).

- Confirmation email to student of the appointment details.

DNA policy - limits to service for chronic non-attendees.

- Email sent if can't reach by phone

- Email in addition to SMS

- Email reminder

Q86 Does the service routinely provide students with information on alternate options for seeking counselling and psychological services and mental health treatment? e.g.from alternative on campus service/s offering welfare support, from a private psychologist, community health centre etc.

Table 90

\begin{tabular}{|c|c|}
\hline \multicolumn{2}{|c|}{ Alternative options for seeking counselling and psychological services } \\
\hline Answer Choices & Number of Institutions \\
\hline No & 3 \\
\hline Yes & 28 \\
\hline
\end{tabular}

If yes, managers were asked to please detail when and how this information is provided. Responses:

- Provided in writing during an appointment; will make referral if necessary.

- Online, in person, in handouts. Refer to private providers, Headspace. The other private provider on-campus with which the University has a service agreement with and will expand the service provided to students through.

- Depending on the presentation the referral will be to an appropriate community support eg SARC, Holyoake etc

- Provided via brochure and/or email and via website.

- Flyers handed out during triage and also flyers on the counters of other reception areas

- On triage questionnaire - phone numbers for community services \& phone lines 
- Brochure provided to all students after initial appointment made

- In session or via phone with our concierge staff

- Referring back to GP for Mental Health Plan with a Private Psychologist or other speciality service e.g. eating disorder clinic, CASA, etc.

- We provide information on external agencies and providers

- If required by the student after being assessed by a counsellor.

- At the time of the appointment students are often encouraged to consult their GP for a referral to a Psychologist under a MHCP.

Information in initial email after appointment booked refers to:

In case of an emergency please contact 000 or go to your nearest emergency department.

For 24hr crisis support please phone Lifeline on 131114.

To access other support services phone Beyond Blue on 1300224636 or access the Beyond Blue website https://www.beyondblue.org.au/get-support/get-immediate-support

- Student Support Advisors are first point of contact and will discuss all counselling options. Approx $50 \%$ of students seen by SSA are referred to UON counselling.

- Due to the brief nature of the service offered, external referrals to GP's for Mental Health Care Plans are made seeking referral to external psychologists for longer-term therapeutic intervention.

- Range of external service providing Mental Health support- eg Headspace or private psychology

- Brochure while student waits for appointment, particularly if appointment is at least 1 week away. During session with counsellor if appropriate.

- If the student does not respond to intake call - the list of alternative support options is provided via email.

- This information is provided based on the client's needs and circumstances. This is often discussed face to face

- As needed within the appointment, of after via a follow-up email, via our webpages, via our online tip sheets, etc.

- Given options at time of appointment (if none available). Website info.

- This is provided as part of the initial consultation. Information also available on the website

- At intake and assessment

- Depending on complexity and severity at first session or close to 6 sessions referral options are provided for external psychological support

- GP referral, link in with other community services

Q87 Is the counselling service able to respond to all requests for counselling appointments within two working weeks all year round?

More than half $(53 \%)$ of the responses identified that 'the counselling service was able to respond to all requests for counselling appointments within two working weeks all year round'. 
Service able to respond to all requests for counselling appointments within two working weeks all year round

\begin{tabular}{|c|c|}
\hline Answer Choices & Number of Institutions \\
\hline No & 15 \\
\hline Yes & 17
\end{tabular}

Managers were asked to describe any specific strategies utilized to follow up students who request an appointment and are not able to be seen within 14 days.

Responses:

- Encourage them to keep presenting. Often we can get new clients in within a few days to a week at most. The ongoing appointments / accessibility is hardest and in busy times can include a wait of 5-6 weeks. We counter that with an increase of on-the-day appointments with all counsellors but this is not ideal.

- Not during the busy times. It may stretch to 3 weeks

- Option for a phone triage conducted by senior counsellor. Option for a 'single' 60 min appt with senior counsellor.

- Students are advised during triage that they may have to wait for an appointment and they should go to their GP or ED if things escalate before their scheduled appointment

- Risk prioritised for urgent clients to be placed into a duty counselling slot asap. If risk is less, but wait is longer, clients are encouraged to attend groups to assist with delay in receiving an individual appt.

- Put on wait list for cancellations. Offered very brief counselling as an alternative (1 to 2 sessions only)

- Cancellation List.

- Academic Liaison Officers may check in with students to follow up while they are waiting. Recommend that student consult with GP and accesses external services.

- Student will be seen by Student Support Advisors within 1-3 days however wait for initial counselling appointments vary 2-5 weeks.

- Brief initial appointments offered and priority appointments kept open for emergent cases.

- If students indicate urgency or concern about having to wait for an appointment we would offer initial support through our Duty Staff to assess and support appropriately and they can fill in an online triage form to help in this process. We also offer same day appointments to manage more concerning presentations and study adjustments that are more time critical.

- Use of no show appointments

- Note - all students are contacted within 2 working days for initial telephone assessment (up to 30mins). Wait until 1st in person (face:face) appt can be 2-4 weeks at peak times, however, alternate options are accessible in the interim.

- Our triage service is available all day Monday - Friday. This is a first come first served basis. If the appts are already full for the day or the student is unavailable, the student is offered a 30min consultation on a different day

- Students are placed on a cancellation list 
- Alternative means of talking with counsellors are emphasised (phone, online etc) but not always acceptable to students. This means they have to wait longer for a preferred face to face. Wait times can be up to a month with a preferred counsellor.

- Initial consultations only released on the day or for the next day. Return appointments are available in consultation with the Counsellor and client.

- All students provided with an initial phone intervention. Students directed to online resources whilst they wait.

- none

- Where able

Q88 Please specify the average wait time for a first appointment or the delay encountered to provide a first consultation after initial request.

Responses: Range one (1) day to one (1) month.

- Approx. 2 days.

- If the student makes it clear it is necessary sooner rather than later, we find a way to prioritise around a week or 2 and this is helped if there is another advocate (eg. residential). If not, in the busy period a wait is regularly longer than a month if they do not access bookon-day.

- 3-10 days

- Not known as no wait list utilised.

- In general within 2 weeks; during peak (mid April-start of exams; Oct-start of exams) 3-4 weeks

- Varies at different times of the year.

Currently 3-4 weeks

- 1 day for our duty counsellor to six weeks for routine non urgent initial assessment

- Between same day and 14 days (calendar). Average 4.4 days.

- Average time is 5 days. However can increase to 10-14 during peak periods

- 5 days

- 2-3 days

- 5 working days. 10 working days in peak periods.

- On the day - 1 week for appointment with SSA

- 1 week

- Approximately 2-3 weeks

- Usually 2 weeks with general services, with individual practitioners what work part-time it can increase to 3 weeks.

- Less than 8 days

- Up to one week

- 3 days

- One week 
- 1-2 days for a triage $/ 30 \mathrm{~min}$ appt. For ongoing counselling post triage, during peak time of university, this can take up to 7 days for the first hour long counselling appt is available

- We can usually respond to a request within 2 weeks, but it increases to 3 weeks during peak times. We try to manage this via a wait/cancellation list where possible, or will offer more short consultations during peak times.

- Peak periods 10 working days. All other times within 5 working days

- 2 weeks

- Approximately 2-4 weeks.

- Initial consultations only released on the day or for the next day. If miss out on the day students try again the next morning from 9am.

- Low period 1 week. High period 2-3 weeks

- All initial enquiries provided with a response within 24-48 hours by phone. Average wait time is 2-4 weeks for first appointment.

- 8 working days

- 2-3 weeks at peak periods

- One to two days depending on the time of semester and demand of service

- One day

Q89 Does your counselling service offer internship placements (e.g.psychology or social work) that extend capacity for service provision?

Only four (4) managers indicated that internships/placements were offered. However the 'other responses' suggest that additional FTE is provided for many services via internships and placements.

Table 92

\begin{tabular}{|c|c|}
\hline \multicolumn{2}{|c|}{ Offer internship placements } \\
\hline Answer Choices & Number of Institutions \\
\hline Yes & 4 \\
\hline No & 14
\end{tabular}

If yes please indicate the additional FTE per annum that these placements add to the service capacity.

\section{Responses:}

- If total uptake, it would be an additional 0.2FTE.

- Varies and restricted by capacity to accommodate (room) for intern. At best FTE 0.4 achieved but option not available every semester.

- Between 1.6FTE-2.4FTE depending availability of supervisors and offices.

- 0.4 , FTE of a counsellor \& of a Psychologist in training. However, not in Centre all year, and can only take a certain (lower) level of risk.

- 0.4

- 2.5

- 0.16 
- 0.2

- $0.4 \mathrm{FTE}$

- 1.0

- 0.8

- Between 1.0 to 2.0 FTE.

- 7 interns x 0.4 (2 days each for 10 months).

- Adds 1.2 FTE.

Q90 Has your institution established roles that support a Safer Community (teams) approach?

Only one (1) manager responded that the institution had established roles that support a Safer Community (teams) approach.

Table 93

Established roles that support a Safer Community (teams) approach

\begin{tabular}{|c|c|}
\hline \multicolumn{2}{|c|}{ Established roles that support a Safer Community (teams) approach } \\
\hline Answer Choices & Number of Institutions \\
\hline Yes & 1 \\
\hline No & 14 \\
\hline
\end{tabular}

Managers were asked to indicate how the Safer Community approach has impacted on the roles, responsibilities and expectations of the counselling service.

Responses:

- Don't know sorry.

- It has had very little impact as the Safer community team understands our limits to confidentiality.

- A work in progress.

- Don't know what this is?

- Manager convenes the risk assessment and advisory committee (threat management).

- We have developed a specific service, Speak Up, that manages this. Counselling work closely around psychological support for students in this situation, support during interviews and student misconduct investigations and secondary consultation. Counselling Manager also provides clinical supervision to Speak Up Staff.

- More involvement in consultations to staff about how to manage specific student issues. Involvement of Head of Counselling in Behavioural Risk Assessment Team process and related duties.

- Currently putting a business case together to establish a Safer Community Unit.

- We have employed a specialist sexual assault counsellor ie someone with a specialised background in counselling, training and supporting victims of assault and harassment. We also have an expectation that all staff record data relating to sexual harassment and assault in a specific format and spreadsheet.

All staff have undertaken First Respondent training - but I note this would be considered standard training ordinarily anyway.

- Most of the safer community activities undertaken by Manager Student Equity and Wellbeing, impact on counselling service staff has been minimal to consultation with Manager. 
- The Safer community officers and CAPS work together in managing difficult/ challenging students that are often in the background of a mental health difficulty.

Referrals received by our service apart from those students who self refer are largely from Safer Community.

- We liaised with the Safety Project Office in HR who developed Emergency Management plans and a Safety App - HR took on board input from our Team. Roles, responsibilities and expectations of our Service have not really changed. We also do not have capacity to respond to matters $24 / 7$.

- Still early days for our institution in establishing a regular meeting of these stakeholders. Initial impact has been around improved tracking of incidents and behaviours of concern across the institution. Stakeholders are starting to agree on combined tools to achieve this and develop associated policy and procedures that better address. This will assist counsellors and being able to respond less ad hoc and more consistently.

- The Safer Community approach works well at our institution as they take care of noncounselling issues to do with safety, misbehaviour, harassment, sexual assault etc. They refer to Counselling appropriately if there are counselling or mental health concerns. Very occasionally we may be asked to accompany them to an incident where there is a clear mental health emergency impacting on the behaviour or situation.

- Close collaboration between Counselling and Safer Community teams fast track referrals to Counselling.

- Contracted Sexual Assault Counsellor@0.2.

- Work closely with SCU staff and they with counselling. This has greatly reduced inappropriate referrals to counselling by security staff and provides significantly better (safer) outcomes for students and the university community as a whole.

Q91 Which of the following psychological/psychotherapeutic orientations are utilised by professional staff working 1-1 or in workshop formats? Tick all that apply.

Managers indicated that CBT, Behavioural and Solution Focused approaches are the most utilised therapeutic frameworks/orientations, followed by Narrative, Schema, Eclectic and Interpersonal Psychotherapy, Psychodynamic, Integrative and Developmental. DBT and Motivational Interviewing were among the other approaches mentioned in additional psychotherapy frameworks used within student counselling services.

Table 94
\begin{tabular}{|c|c|}
\hline Psychological/psychotherapeutic orientations are utilised by professional staff \\
\hline Answer Choices & Number of Institutions \\
\hline Cognitive - Behavioural (e.g. CBT) & 32 \\
\hline Behavioural (e.g. ACT) & 31 \\
\hline Solution-focused & 30 \\
\hline Narrative & 20 \\
\hline Schema focused & 20 \\
\hline Eclectic & 18 \\
\hline Interpersonal Psychotherapy & 14 \\
\hline Psychodynamic & 14 \\
\hline Integrative & 13 \\
\hline Developmental & 11 \\
\hline Other (please specify) & 7 \\
\hline
\end{tabular}

Other therapeutic frameworks were identified: 
- $\quad$ MBT, DBT (I know that could fit in CBT), motivational interviewing

- Primarily CBT and ACT (behavioural)

- DBT

- EMDR

- DBT

- Motivational Interviewing

- Mentalisation

- EMDR, and trauma-focussed approaches.

- Sandplay.

Q92 Are service practitioners routinely using a client outcome measure (e.g. OQ 45.2 or ORS/SRS scales)?

Few managers $(\mathrm{N}=5)$ reported the use of client outcome measures as standard service procedure. Fourteen (14) reported that use of client outcome measures did occur but at the counsellor's choice. Thirteen (13) managers reported that none of the counsellors used client outcome measures. Measures used include the OQ45, the Rosenburg (in sessions 1, 3 and 6), the ORS/SRS and the K10 and DASS as being trialled as pre and post tests.

Table 95

\begin{tabular}{|l|c|}
\hline \multicolumn{1}{|c|}{ Answer Choices } & $\begin{array}{c}\text { Number of } \\
\text { Institutions }\end{array}$ \\
\hline Yes - use of outcome measures are standard service procedure & 5 \\
\hline Yes - some but not all - use of a client outcome measure is counsellor's choice & 14 \\
\hline None of the counsellors use a client outcome measure & 13 \\
\hline
\end{tabular}

Specified outcome measure/s being used:

- OQ45 \& Rosenburg in session 1,3 and 6

- OQ 45.2 TA

- Yes, only during one week each semester SRS scale

- Trialling the use of the DASS21 at one campus with intention to adopt across all sites.

- K-10 and DASS (Pre and post)

- ORS/SRS but only 1-2 counsellors would occasionally use these

- $\mathrm{ORS} / \mathrm{SRS}$

Q93 Does the service utilise the CCAPS as a pre/post assessment screen? Reference:

http://ccmh.psu.edu/ccaps-instruments/

Only one (1) manager reported using the CCAPS as a pre/post assessment screen.

Table 96

\begin{tabular}{|c|c|}
\hline \multicolumn{2}{|c|}{ Utilise the CCAPS as a pre/post assessment screen } \\
\hline Answer Choices & Number of Institutions \\
\hline No & 30 \\
\hline Yes & 1
\end{tabular}

Managers using CCAPS were asked to state how the CCAPS is delivered. E.G. within Point and Click or Titanium software or other (please specify). 
Response provided:

- Upon arrival at first appointment through an internal database (SMP).

Q94 Does your service utilise a 'consumer or student reference group' to inform and guide service delivery?

The majority of managers $(\mathrm{N}=25)$ did not use a consumer or student reference group. Seven (7) managers reported utilising a 'consumer or student reference group' to inform and guide service delivery.

\begin{tabular}{|c|c|}
\hline \multicolumn{2}{|c|}{$\begin{array}{c}\text { Use of a 'consumer or student reference group' to inform and guide service } \\
\text { delivery }\end{array}$} \\
\hline Answer Choices & Number of Institutions \\
\hline No & 25 \\
\hline Yes & 7 \\
\hline
\end{tabular}

Managers using a student reference or consumer group were asked to specify how students were recruited.

The following responses were provided:

- Though the students association does provide feedback generically to the University.

- via student rep groups on campus plus student wellbeing team members

- But this is currently being explored.

- Students who are on the Council of the Student Association are sometimes invited to provide input into service improvements. Students can also be recruited via social media produced by the Student Association and the institution.

- Drug, Alcohol and other Addiction service utilise regular focus groups and tend to recruit via student residents

- The University has a Student Advisory Committee with a combination of student representatives nominated by Faculty, self-nomination and Student Association. Mental Health and availability of services features in the agenda of the bi-yearly forums.

- Somewhat - student representatives attend a student services feedback meeting, which sometimes informs counselling service.

- No, but we are planning to implement in 2018.

- We have a number of students on a number of different groups that can provide feedback and inform service delivery. These groups are the Mental Health Promotion and Strategy Working Group, Mental Health Consultant's Group and the Health Ninja's, the later two are run via the Health Promotion Coordinator's office, separate to Counselling.

- Surveys

Q95 Does the service employ other methods of seeking student/stakeholder input to service delivery planning?

The majority of managers $(\mathrm{N}=20)$ use other methods than those identified in Q94 to seek student/stakeholder feedback. The responses indicate that stakeholder consultation and consumer feedback is a high priority for heads of counselling services. Surveys, focus groups, regular meetings with student groups and Faculty members, formal Work Integrated Learning (WIL) activities, Students as Partners initiatives and formal student partnership models are all identified as are partnerships with the residential college communities. 
Table 98

\begin{tabular}{|c|c|}
\hline \multicolumn{2}{|c|}{ Other methods used to seek student/stakeholder input to service delivery planning } \\
\hline Answer Choices & Number of Institutions \\
\hline No & 11 \\
\hline Yes & 20 \\
\hline
\end{tabular}

Managers were asked to describe the methods used and comment on benefits/lack of value inherent in the approach. The following responses were provided:

- Annual survey across one regular month. Ad-hoc discussion with student body.

- Feedback surveys

- Students involved in WIL or Students as Partners in some of the service activities

- Individual feedback during /post sessions

- Regular surveys and focus groups held

- Focus groups - student interest sought through social media posts and student union

- Looking at developing a formal student partnership model Student satisfaction survey run twice a year. Consultation from Queer Peer Mentors.

- We meet regularly with AUSA - the Student Body

- Student will sit on some advisory groups e.e. Psychological Health Working Group, Health Promotion Working Group and Sexual Misconduct Working Group

- Occasional student satisfaction surveys. Student Representative Council feedback

- NPS

- Meetings with student body / doctors

- For services delivered to students living in the on-campus residential system, we consult with Heads of Colleges, as well as student leaders re: their needs. We also consult with the Students' Association on campus, and liaise with other student support service staff to gauge what needs they are seeing. We are also guided by ad hoc client feedback.

- Send out the client feedback questionnaire annually - only permitted to send this survey out once a year by senior management.

- Client surveys

- Some consultation through key committees (eg the Student Experience Committee), which has student and staff reps.

Consultation with student union reps on an annual basis.

User surveys

All methods provide some helpful feedback but often unrealistic expectations expressed.

- Our Faculties are stakeholders and they input into our performance in meetings for as student services twice a year

- Direct consultation with Student Association (Guild) board

- Consultation with Student Association and student council. Internal intranet.

- Regular meetings with student organisation and faculty stakeholders

Q96 How frequently does your service conduct a 'client satisfaction' survey?

The majority of managers reported using client satisfaction surveys. Only two (2) responders stated 
that they had not ever used a client satisfaction survey. The most common frequency for deploying a client satisfaction survey was 'once a year' $(\mathrm{N}=11)$. Eighteen (18) managers used client satisfaction surveys less frequently (occasionally, for strategic planning/review process, every 2,3, or 5 years).

Table 99

\begin{tabular}{|l|c|}
\hline \multicolumn{1}{|c|}{ Client satisfaction survey } \\
\hline \multicolumn{1}{|c|}{ Answer Choices } & Number of Institutions \\
\hline Yes once a year & 7 \\
\hline $\begin{array}{l}\text { Occasionally - institution has restrictions on number of student surveys } \\
\text { conducted }\end{array}$ & 5 \\
\hline Yes every three to five years & 5 \\
\hline Yes for specific purposes (strategic planning, review process etc) & 1 \\
\hline Yes every two years & 2 \\
\hline Not ever & \\
\hline
\end{tabular}

Q97 Please describe any other routine feedback, evaluation or quality measures used by your service.

Managers were asked to comment on other routine feedback, evaluation or quality measures used within their service. The following ten (10) responses were received:

- There are flyers at reception that students can complete voluntary

- We conduct the survey twice a year.

- Regular evaluation of service usage statistics.

- Student are sent evaluation surveys after initial, 3 and 5th session, however student do not regular complete them. This needs to be reviewed and updated.

- N/A

- Feedback sought through the University's Student Feedback mechanisms.

- Net promoter score telephone interviews by random selection.

- See answer to Q 94. We also routinely ask our clients within any given appointment if their needs are being met. We also collect workshop evaluation forms.

- We run a client satisfaction survey twice a year for all clients who come in May and October. All workshop participants also fill out satisfaction feedback surveys.

- Survey

Q98 Has your service or institution conducted any research on student mental health or student well-being between 2015-2017?

Managers were asked to identify any research conducted on student mental health or student wellbeing between 2015 and 2017. Seven (7) managers reported relevant research in this time frame.

Table 100

\begin{tabular}{|c|c|}
\hline Conducted any research on student mental health or student well-being between 2015-2017 \\
\hline Answer Choices & Number of Institutions \\
\hline Yes & 7 \\
\hline No & 6 \\
\hline Not applicable & 19 \\
\hline
\end{tabular}




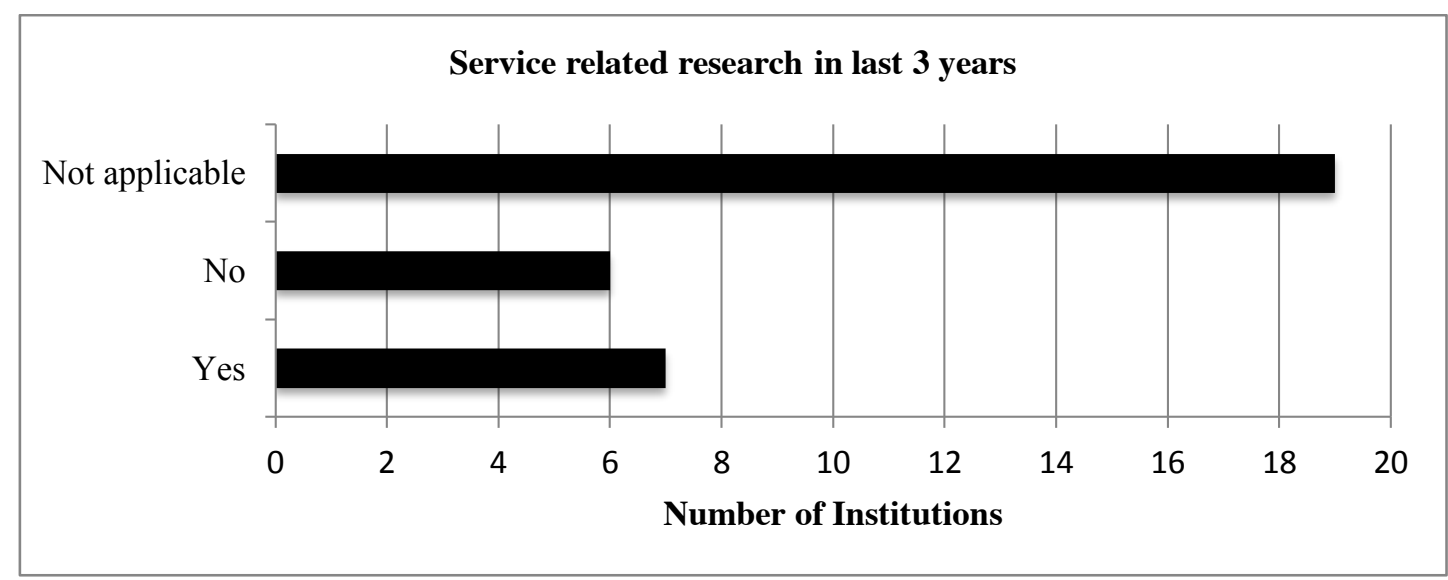

Figure 23

Q99 Is there a research report or publication that can be accessed by other Heads of Counselling Services?

Nine (9) responses were received:

1. Sydney Uni: Student preparedness for University Survey. Report Available. philomena.renner@sydney.edu.au

2. UTAS: Specific to preparation programs - Emotional labour demands in enabling education: A qualitative exploration of the unique challenges and protective factors. Student Success, Vol 9 Issue 1, Feb 2018. Colin.Clark@utas.edu.au

3. UNE: evaluated the needs of students on the Autism Spectrum, and evaluated a peer mentoring program delivered in 2015. The results of this have been written up but are just being finalised for publication. Also published an article on the sexual health needs of students. asteven2@une.edu.au

4. Macquarie Uni: UniFIT - combining psycho-ed with exercise sessions UniWellbeing online treatment program. No report ben.wilkes@mq.edu.au

5. UON: Student Health Lifestyle Survey (conducted every 2 years) Student Feedback on UON (conducted every 2 years) Commencing Student Survey SSAF Student Survey. jacqueline.olley@hotmail.com

6. CQU: Current research project being undertaken by Queensland Centre for Domestic Violence and CQU to explore students experiences of sexual harassment and assault. (Will share findings with the group once reported). No Report. b.mackay@cqu.edu.au

7. LaTrobeUni: Joint project currently underway between Counselling, Post Graduation Research School and Student Advocacy looking at wellbeing and mental health issues with our Post Graduate students. No Report. L.DuPlooy@latrobe.edu.au

8. Victoria University Wellington NZ: have conducted annual survey of all students' emotional wellbeing using the WHO 5 wellbeing measure for the past five years. No Report. gerard.hoffman@vuw.ac.nz

9. Melbourne Uni: Health Information Survey was run in 2017 by the Health Promotion Coordinator. The Centre for Study of Higher Education has run a large 8 Faculty student wellbeing survey. Report available. orania@unimelb.edu.au

Q100 During 2017 has your service directly charged a fee to any student for counselling and psychological services

One (1) manager indicated that a fee had been charged for counselling and psychological services. The majority $(\mathrm{N}=31)$ stated that no fee had been charged for counselling and psychological services. 
Table 101

\begin{tabular}{|c|c|}
\hline Charged a fee to any student for counselling and psychological services \\
\hline Answer Choices & Number of Institutions \\
\hline No & 31 \\
\hline Yes & 1 \\
\hline
\end{tabular}

The manager* reporting a fee charge gave the following detail:

Domestic students charged $\$ 8$ and SS insurance for international

* NZ institution

Q101 Type of Fees charged. Please tick all categories below that apply to the counselling service activities.

The majority of managers $(\mathrm{N}=28)$ indicated that no fees were charged for services. One (1) response was received for 'fees charged for 1-1 services. Two responses were received for each of the following:

- Fees charged for cancellation or 'no show' appointments

- Fees charged for services delivered at special request by Faculty or Department

- Fees charged for services delivered to students enrolled with independent entity related to the institution

One (1) response was received for: fees charged for services delivered under a formal MOU.

Table 102

\begin{tabular}{|l|c|}
\multicolumn{1}{|c|}{ Answer Choices } & $\begin{array}{c}\text { Number of } \\
\text { Institutions }\end{array}$ \\
\hline \multicolumn{1}{|c|}{ Ansed } & 28 \\
\hline No fees charged & 1 \\
\hline Fees charged for 1-1 services & 2 \\
\hline Fees charged for cancellation or 'no show' appointments & 2 \\
\hline Fees charged for services delivered at special request by Faculty or Department & 2 \\
\hline $\begin{array}{l}\text { Fees charged for services delivered to students enrolled with independent entity } \\
\text { related to the institution }\end{array}$ & 1 \\
\hline Fees charged for services delivered under a formal MOU & 0 \\
\hline Fees charged for group services & 0 \\
\hline Fees charged for additional services delivered as part of curriculum & 1 \\
\hline Not Applicable & 2 \\
\hline Other (please specify) & 2 \\
\hline
\end{tabular}

Other fees specified were:

- Fee charged to Staff for MHFA training

- This would be for something on top of what is usually provided to all, e.g. a special extra MHFA workshop just for their students

Q102 If fees are charged pleased tick any category offee below that is applicable.

Various fees were being charged. See Table 103. 
Table 103

\begin{tabular}{|l|c|}
\hline \multicolumn{1}{|c|}{ Answer Choices } & $\begin{array}{c}\text { Number of } \\
\text { Institutions }\end{array}$ \\
\hline \multicolumn{1}{|c|}{ Anarged detail } & 3 \\
\hline A cancellation or no show fee of less than \$20 & 1 \\
\hline A cancellation or no show fee of more than \$20 & 1 \\
\hline A token fee for service of less than \$10 & 1 \\
\hline A cost recovery fee of between \$30 and \$90 & 1 \\
\hline Full fee charged based on professional association recommended rate & 1 \\
\hline $\begin{array}{l}\text { Staff using the counselling services to access 1-1 psychological services are } \\
\text { charged as per health insurance/Medicare schedule as applicable }\end{array}$ & 1 \\
\hline $\begin{array}{l}\text { Faculties are charged for specific requested services such as leadership } \\
\text { workshops for specific student cohorts, within curriculum lectures, workshops } \\
\text { delivered to specific student cohorts etc }\end{array}$ & \\
\hline Fees are charged but none in the options listed above & 1 \\
\hline A university subsidized fee of less than \$30 & 0 \\
\hline A university subsidized fee of more than \$30 & 0 \\
\hline A student association subsidised fee of less than \$30 & 0 \\
\hline A student association subsidised fee of more than \$30 & 0 \\
\hline A cost recovery fee of more than \$90 & 0 \\
\hline Full fee charged based on your services/institutions fee scale & 0 \\
\hline Not applicable & 13 \\
\hline Other (please specify) & 1 \\
\hline Other types of fees charged: & \\
\hline
\end{tabular}

Other types of fees charged:

- Fees could be charged for something that is beyond the usual offering and happens very rarely, e.g. a Department or School asks for an extra 2 day MHFA training program just for their students, we would charge for that.

\section{Q103 With regards to service fees which of the following options are currently under consideration?}

The majority of managers $(\mathrm{N}=23)$ were not considering a fee introduction. Four (4) managers were considering charging a cancellation or 'no show' fee. One (1) manager was considering charging for requested services by Faculties, private residential colleges, institution associated entities. Three (3) managers indicated that fees were under consideration. The responses are detailed below and include charging under Medicare, outsourcing and using a blended model. Charging for training delivered is also mentioned.

Table 104

\begin{tabular}{|l|c|}
\multicolumn{1}{|c|}{ Fees under consideration } \\
\multicolumn{1}{|c|}{ Answer Choices } & $\begin{array}{c}\text { Number of } \\
\text { Institutions }\end{array}$ \\
\hline Not considering any fee introduction & 23 \\
\hline Considering a small cancellation or no show fee & 4 \\
\hline $\begin{array}{l}\text { Considering charging for requested services by Faculties, private residential } \\
\text { colleges, institution associated entities }\end{array}$ & 1 \\
\hline Considering token fee & 0 \\
\hline Considering a subsidized fee & 0 \\
\hline Considering a cost recovery fee & 0 \\
\hline Considering professional association recommended fee & 0 \\
\hline None of the above & 3 \\
\hline Other (please specify) & 3 \\
\hline
\end{tabular}

Other responses: 
- In early discussion. Looking at Medicare model. Outsourcing services to make it more 'costeffective' is being blended at the moment.

- Fee for training i.e. MHFA, Residential Assistants training

- We already charge for services beyond an orientation briefing to residential colleges, and also on something beyond the normal delivery for Faculties.

\section{Q104 Medicare options: Please tick any of the following that apply:}

The majority of managers $(\mathrm{N}=22)$ reported that no salaried counsellors use Medicare options to charge students. Seventeen (17) managers indicated that 'some' or 'many' students likely to be eligible for psychological treatment under Medicare funded options are referred to their general practitioner or the general practitioners in the university health'. One (1) response indicated that it was service policy to refer students likely to be eligible for psychological services under Medicare to be 'referred to their general practitioner or the general practitioners in the university health service'. One (1) response indicated that 'students are referred to service professional staff under the Medicare funded options by their general practitioners and these students are bulk billed only'. One (1) other response indicated that 'independently practicing psychologists with Medicare rebate options have rooms within the service and are the only practitioners charging fees within this service'. Three (3) responses provided additional information. See 'other responses' below.

Table 105

\begin{tabular}{|c|c|}
\hline \multicolumn{2}{|l|}{ Medicare rebate use } \\
\hline Answer Choices & Number of Institutions \\
\hline $\begin{array}{l}\text { No salaried professional staff are utilising Medicare options to charge } \\
\text { students within this service }\end{array}$ & 22 \\
\hline $\begin{array}{l}\text { Some students likely to be eligible for psychological treatment under } \\
\text { Medicare funded options are referred to their general practitioner or the } \\
\text { general practitioners in the university health service }\end{array}$ & 12 \\
\hline $\begin{array}{l}\text { Many students likely to be eligible for psychological treatment under } \\
\text { Medicare funded options are referred to their general practitioner or the } \\
\text { general practitioners in the university health service }\end{array}$ & 5 \\
\hline $\begin{array}{l}\text { It is service policy that ALL students likely to be eligible for psychological } \\
\text { treatment under Medicare funded options are referred to their general } \\
\text { practitioner or the general practitioners in the university health service }\end{array}$ & 1 \\
\hline $\begin{array}{l}\text { Students are referred to service professional staff under the Medicare funded } \\
\text { options by their general practitioners and these students are bulk billed only }\end{array}$ & 1 \\
\hline $\begin{array}{l}\text { Salaried professionals within the service are billing students under the } \\
\text { Medicare options with the Medicare rebate signed over to the service }\end{array}$ & 1 \\
\hline $\begin{array}{l}\text { Independently practicing psychologists with Medicare rebate options have } \\
\text { rooms within the service and are the only practitioners charging fees within } \\
\text { this service }\end{array}$ & 1 \\
\hline $\begin{array}{l}\text { Students are referred to service professional staff under the Medicare funded } \\
\text { options by their general practitioners and these students are charged the } \\
\text { amount funded under Medicare plus an additional amount }\end{array}$ & 0 \\
\hline Other (please specify) & 3 \\
\hline
\end{tabular}

Other responses:

- The private provider is in the same building but not in our service. They are bulk-billing with students.

- Independently practicing psychologists with Medicare rebate options have rooms managed by the Health Service. NewAccess coaching is provided on campus one day a week using a room located in the Health Service. 
- My understanding is that if we are salaried staff, funded by the Gov't to provide psychological services, that it is then not possible to also get Medicare funding - as this is deemed "double dipping".

Q105 Does the service currently provide any counselling and psychological services (1-1 or in group) to students via Medicare bulk billed options?

The majority of managers $(\mathrm{N}=24)$ responded that services were not provided to students under Medicare bulk-billing. Three (3) managers responded that services were currently provided to students under Medicare bulk-billing.

Table 106

\begin{tabular}{|c|c|}
\hline \multicolumn{2}{|c|}{ Current use of Medicare bulk-billing } \\
\hline Answer Choices & Number of Institutions \\
\hline No & 24 \\
\hline Yes & 3 \\
\hline
\end{tabular}

Q106 Is your service actively considering providing students with direct access to bulk bill services under Medicare items?

Four (4) managers indicated that they were 'actively considering providing students with direct access to bulk bill services under Medicare items'.

Table 107

\begin{tabular}{|c|c|}
\hline \multicolumn{2}{|c|}{$\begin{array}{c}\text { Service actively considering providing students with direct access to bulk bill } \\
\text { services under Medicare items }\end{array}$} \\
\hline Answer Choices & Number of Institutions \\
\hline No & 22 \\
\hline Yes & 4 \\
\hline
\end{tabular}

Managers were asked to explain the model they are considering for providing access to Medicare funded services. The following three (3) comments were received:

- Our service isn't. Our managers are indicating they are.

- Already do this. First 3 outside of Medicare and then to GP for MHCP ax.

- Contractor Medicare Psychology

Q107 Are students able to have access to private psychologists or social workers who bulk bill under Medicare items via another university provided service such as a medical service?

Nine (9) responses were received in the affirmative indicating that students were able to have access to private psychologists or social workers who bulk bill under Medicare items via another university provided service such as a medical service. The majority of responses $(\mathrm{N}=17)$ indicated that students were not able to have access to private psychologists or social workers who bulk bill under Medicare items via another university provided service such as a medical service.

Table 108

Students able to have access to private psychologists or social workers who bulk bill under Medicare items via another university provided service such as a medical service

\begin{tabular}{|c|c|}
\hline Answer Choices & Number of Institutions \\
\hline No & 17 \\
\hline Yes & 9
\end{tabular}

Managers were asked to explain the model they are considering for providing access to Medicare funded services. The following comments were received:

- Our previously owned University medical centre has been outsourced to a private provider. They are on campus and provide access for bulk-billing psychs in that service. 
- Via Mental Heath Nurse in Health Service and via psychologists working out of rooms provided by the Health Service/University.

- Psychology Clinic

- The University Health Service has bulk-billing psychologist.

- Not under Medicare, but we do pay for sessions at the intern clinical psychology clinic for students to attend up to 13 sessions in a year.

- GP's will refer students to external psychologists who bulk bill

- If a GP within our Uni Medical Centre chooses to refer a student to a private practitioner who bulk bills, then the student may wish to take up that option.

- University Health Service (UHS)

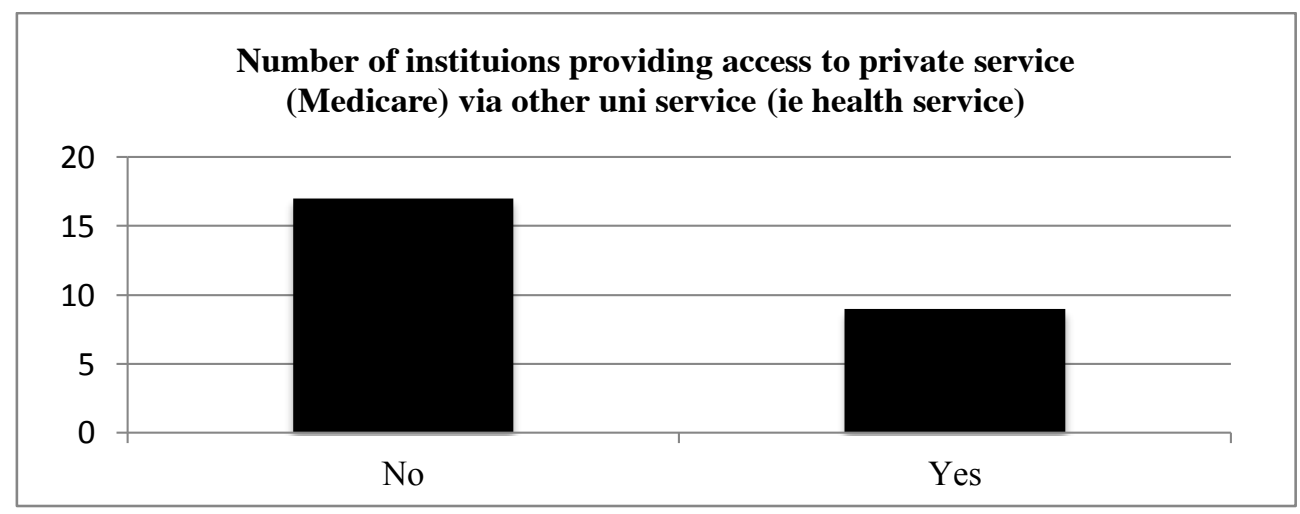

Figure 24

Q108 Does your service provide counselling and psychological services to students enrolled at another institution under a formal MOU agreement?

The majority of managers $(\mathrm{N}=23)$ replied that the service did not provide counselling and psychological services to students enrolled at another institution under a formal MOU agreement. Eight (8) managers responded that the service did provide counselling and psychological services to students enrolled at another institution under a formal MOU agreement.

Table 109

Provide counselling and psychological services to students enrolled at another institution under a formal MOU agreement

\begin{tabular}{|c|c|}
\hline Answer Choices & Number of Institutions \\
\hline No & 23 \\
\hline Yes & 8 \\
\hline
\end{tabular}

Managers were asked to detail if the MOU arrangement was primarily cost recovery or if there was a built in profit margin. Most indicated cost recovery. The following comments were received:

- Primarily cost recovery.

- Cost recovery

- Small profit margin

- No recovery cost

- We can provide services to ANU student who are enrolled in Joint Medical program

- Cost recovery 
- Built in profit margin

Q109 Does your service charge other areas of your institution for services delivered that go beyond your core services? e.g. mental health awareness training for staff, leadership programs for elite students etc.

Seven (7) managers indicated that the service did service charge other areas of the institution for services delivered that go beyond your core services. The majority $(\mathrm{N}=25)$ indicated that the service did not charge other areas of the institution for services delivered that go beyond your core services.

Table 110

\begin{tabular}{|c|c|}
\hline $\begin{array}{c}\text { Charge other areas of your institution for services delivered that go beyond } \\
\text { your core services }\end{array}$ \\
\hline Answer Choices & Number of Institutions \\
\hline No & 25 \\
\hline Yes & 7 \\
\hline
\end{tabular}

Q110 Does your service undertake any pro bono service delivery for an institution affiliated with your institution?

Eight (8) responses indicated that the service did undertake pro bono service delivery for an affiliated institution. The majority $(\mathrm{N}=24)$ were 'no' responses.

Table 111

Service undertakes pro bono service delivery for an affiliated institution

\begin{tabular}{|c|c|}
\hline Answer Choices & Number of Institutions \\
\hline No & 24 \\
\hline Yes & 8 \\
\hline
\end{tabular}

Q111 Does your service offer provision for 'outsourced' options for counselling or psychological services via private practitioners (who on arrangement will invoice your service) to students who are undertaking an academic program placement or workplace internship etc, in overseas, rural or regional locations?

Eleven (11) responses indicated that 'occasionally' $(\mathrm{N}=6)$ or 'regularly' $(\mathrm{N}=5)$ the service offered 'outsourced' options for counselling or psychological services via private practitioners (who on arrangement will invoice the service) to students who are undertaking an academic program placement or workplace internship etc, in overseas, rural or regional locations. The majority of responses $(\mathrm{N}=21)$ indicated that such provision was 'not ever' available.

Table 112

\begin{tabular}{|c|c|}
\hline $\begin{array}{c}\text { Provide 'outsourced' options for counselling or psychological services via private } \\
\text { practitioners (who on arrangement will invoice your service) to students who are } \\
\text { undertaking an academic program placement or workplace internship etc, in overseas, } \\
\text { rural or regional locations }\end{array}$ \\
\hline $\begin{array}{c}\text { Response } \\
\text { Not ever }\end{array}$ & 21 \\
\hline Occasionally if no other option available & 6 \\
\hline Regularly as part of routine service delivery & 5 \\
\hline
\end{tabular}

Q112 How many students in total were eligible to receive services from the counselling service at your institution in 2017?

In 2017 the number of students, who were eligible to receive services from the twenty-nine counselling services at responding institutions that provided data, was $\mathrm{N}=888,166$.

The range of responses across the twenty-nine (29) institutions $=\sim 400$ to 67,000 . The number of students entitled to access the counselling service most commonly indentified ( $N=9)$ was $>20,000$ - 
$30,000$.

Table 113

\begin{tabular}{|c|c|}
\hline $\begin{array}{c}\text { Students eligible to receive services from counselling } \\
\text { service in 2017 }\end{array}$ & $\begin{array}{c}\text { Number of } \\
\text { Institutions }\end{array}$ \\
\hline N/A & 2 \\
\hline$<500-10,000$ & 3 \\
\hline$>10,000-20,000$ & 4 \\
\hline$>20,000-30,000$ & 9 \\
\hline$>30,000-40,000$ & 6 \\
\hline$>40,000-50,000$ & 1 \\
\hline$>50,000-60,000$ & 3 \\
\hline$>60,000-<70,000$ & 3 \\
\hline
\end{tabular}

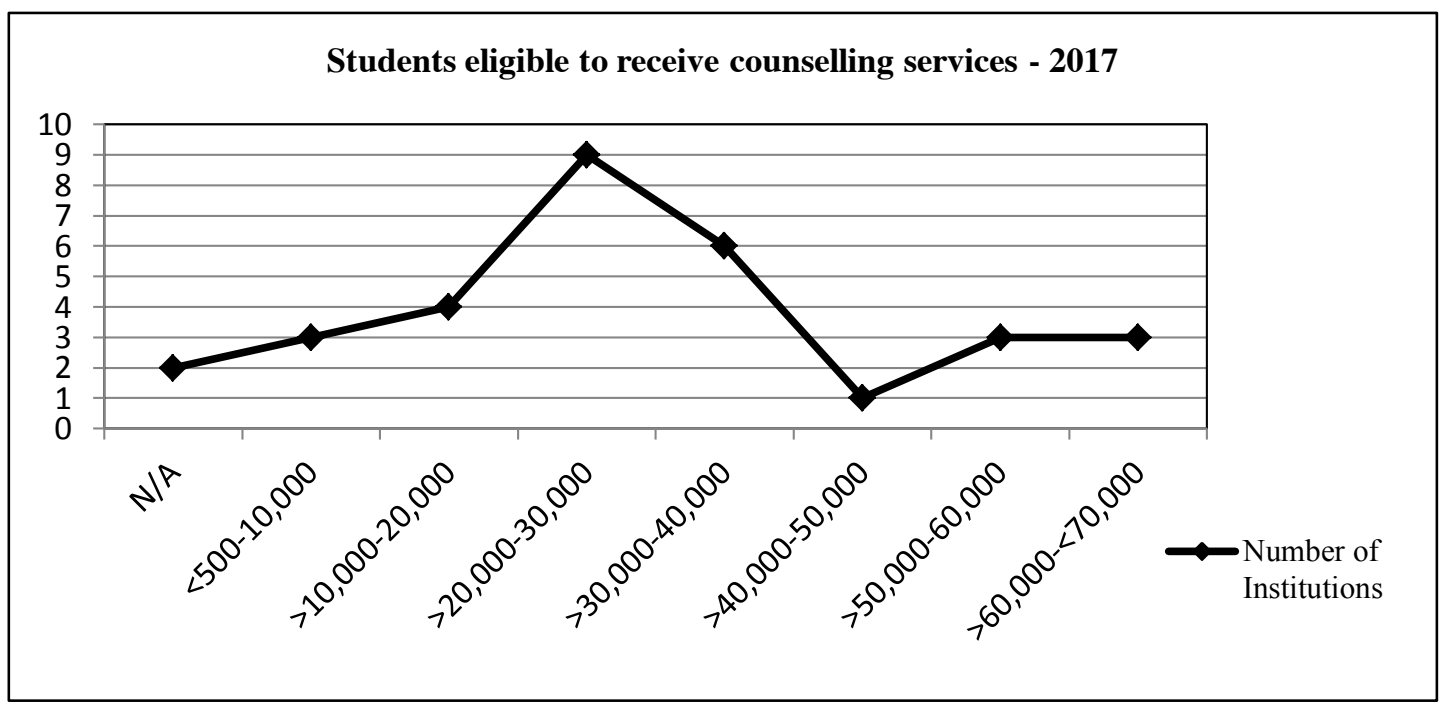

Figure 25

Q113 What percentage of students who were eligible for 1-1 counselling services actually accessed 1-1 services in 2017 ?

Four (4) responders indicated this data was not available.

The range of responses from the twenty-seven (27) managers that returned specified percentages varied from $2.5 \%$ to $70 \%$. (See comments on * and ** below).

Ten (10) responses indicated the percentage of eligible students accessing the 1-1 counselling services during 2017 was between $2.5 \%$ and $5 \%$. A further six (6) responses returned percentages between $>5-10 \%$. Seven (7) responses reported percentages between $>10 \%-15 \%$. Two (2) responses reported percentages between $>20-30 \%$. One (1) response reported $35 \%$, which the responder declared, was 'inferred' and one (1) reported a percentage of $70 \%$. See * and ** below for further comment. 
Table 114

\begin{tabular}{|c|c|}
\hline $\begin{array}{c}\text { Percentage of eligible students } \\
\text { access 1-1 counselling -2017 }\end{array}$ & Number of Institutions \\
\hline N/A & 4 \\
\hline$>2 \%-3 \%$ & 1 \\
\hline$>3 \%-4 \%$ & 4 \\
\hline$>4 \%-5 \%$ & 5 \\
\hline$>5 \%-6 \%$ & 0 \\
\hline$>6 \%-7 \%$ & 1 \\
\hline$>7 \%-8 \%$ & 4 \\
\hline$>8 \%-9 \%$ & 0 \\
\hline$>9 \%-10 \%$ & 1 \\
\hline$>10 \%-11 \%$ & 1 \\
\hline$>11 \%-12 \%$ & 2 \\
\hline$>12 \%-13 \%$ & 1 \\
\hline$>13 \%-14 \%$ & 1 \\
\hline$>14 \%-15 \%$ & 2 \\
\hline $320 \%-30 \%$ & 2 \\
\hline $70 \% * *$ & 1 \\
\hline & 1 \\
\hline
\end{tabular}

*A single response of $35 \%$ was declared as 'inferred' by the responding manager.

**Given the enormous difference from all other responses it is plausible that the $70 \%$ figure was either an entry error or a calculation error. This response was further investigated and then recalculated using data, and a statement provided by the same responder in Q112 resulting in a new figure of $7.89 \%$. If allocated to the $>7-8 \%$ category in Table 114 (above) the total number of institutions within this response range would be $\mathrm{N}=5$.

Q114 What percentage of students participated in all programs, workshops etc (other than individual 1-1 counselling) during 2017?

The majority of responders $(\mathrm{N}=21)$ indicated that this data was not available $(\mathrm{N} / \mathrm{A})$. The percentage of students that participated in all programs, workshops etc (other than individual 1-1 counselling) during 2017 ranged from $0.3 \%$ to $24.4 \%$. Seven (7) responses were received for categories between $<1 \%$ to $5 \%$. A single response (1) was received for each of the following categories: $>5 \%$ to $10 \%$, $>10 \%$ to $15 \%$ and $>15 \%$ to $25 \%$.

Table 115

\begin{tabular}{|c|c|}
\hline \multicolumn{2}{|c|}{ Students attending group and other programs - 2017 } \\
\hline Percentage of enrolled students & Number of Institutions \\
\hline N/A & 21 \\
\hline $0-1$ & 3 \\
\hline$>1$ to 3 & 2 \\
\hline$>3$ to 5 & 2 \\
\hline$>5$ to 10 & 1 \\
\hline$>10$ to 15 & 1 \\
\hline$>15$ to 25 & 1 \\
\hline
\end{tabular}

Q115 Across service delivery sites (e.g.campuses) how many 1-1 occasions of service did students utilise in 2017?

The range of responses for number of 1-1 occasions of service delivered in 2017 varied from $<1,000$ to $>10,500$. The most common number of $1-1$ occasions of service reported for the $\sim 5,000$ 5,600 category $(\mathrm{N}=5)$. The second most common category reported was $22,300-2,500(\mathrm{~N}=4)$. 
Three (3) responses were received for the $\sim 6,100-6,900$ category. There was one (1) response for category $<1,000$ and two (2) responses for each of the following categories: 1,200-1,300; 3,000 $<4,000 ; \sim 7,000-7,700 ; \sim 8,500-9,000$ and $>10,500$.

Table 116

\begin{tabular}{|c|c|}
\hline \multicolumn{2}{|c|}{ Occasions of service } \\
\hline Number of 1-1 occasions of service & Number of Responses \\
\hline$<1,000$ & 1 \\
\hline$\sim 1,200-1,300$ & 2 \\
\hline$\sim 2,300-2,500$ & 4 \\
\hline$\sim 3,000-<4,000$ & 2 \\
\hline$\sim 5,000-5,600$ & 5 \\
\hline$\sim 6,100-6,900$ & 3 \\
\hline$\sim 7,000-7,700$ & 2 \\
\hline$>8,500-9,000$ & 2 \\
\hline$>10,500$ & 2 \\
\hline
\end{tabular}

The number of 1-1 occasions of service delivered is likely impacted by the counsellor FTE for the service. To explore the relationship between Service FTE, 1-1 occasions of service and enrolment size the data was interrogated and matched for the relevant questions.

Data in Table 117 shows the related data for 31 responders (institutions) ordered by: Service FTE / Enrolment cohort size / Occasions of Service.

To show another comparative view Table 118 shows the same data ordered by: Enrolment cohort size / Service FTE / Occasions of Service.

The highest number of 1-1 occasions of service $\left(\mathrm{N}=10,844^{*}\right)$ was reported by an institution with fewer enrolled students, eight (8) fewer FTE than the next highest reported $(\mathrm{N}=10,779 * *) 1-1$ occasions of service delivered at an institution with approximately 10,000 more enrolled students. The third highest reported 1-1 occasions of service $(\mathrm{N}=8,952)$ was delivered by 1.8 FTE more than the service with the highest reported occasions of service.

However, this comparison fails to establish an understanding of service efficiency without comparing severity of student mental health at presentation, the number of 1-1 occasions of service utilised per student, the policy for referral and related action, the focus on early intervention versus remedial interventions and containment of symptoms prior to referral for those with severe and very severe symptom presentation. These comparisons are beyond the scope of this survey and report.

Table 117

\begin{tabular}{|c|c|c|c|}
\hline Institution \# & Service FTE & Occasions of service & Enrolment cohort size \\
\hline A & 18 & $10,779^{* *}$ & 63,406 \\
\hline B & 13.4 & 6,839 & 40,000 \\
\hline C & 13 & $\sim 7,000$ & 38,000 \\
\hline D & 12.6 & 8,500 & 66,928 \\
\hline E & 12.4 & N/A & 37,531 \\
\hline F & 12 & N/A & 67,000 \\
\hline G & 11.8 & $8,952^{* * *}$ & 60,000 \\
\hline H & 11.2 & N/A & 36,746 \\
\hline I & 10 & $10,844 *$ & 51,338 \\
\hline J & 10 & 6,800 & 23,000 \\
\hline K & 10 & N/A & N/A \\
\hline L & 8.8 & 4,861 & 52,331 \\
\hline M & 8 & 5,492 & 30,767 \\
\hline N & 7.9 & 3,532 & 26,975 \\
\hline
\end{tabular}

Journal of the Australian and New Zealand Student Services Association: 


\begin{tabular}{|c|c|c|c|}
\hline Institution \# & Service FTE & Occasions of service & Enrolment cohort size \\
\hline O & 7.9 & 7,640 & 22,736 \\
\hline P & 7.4 & 5,601 & 23,109 \\
\hline Q & 7 & 5,152 & 44,208 \\
\hline R & 6.8 & 5,589 & 24,000 \\
\hline S & $5.2+1.2$ locums in peak & 6,081 & 27,000 \\
\hline T & 5.1 & 2,457 & 12,000 \\
\hline U & 5 & 2,561 & 31,114 \\
\hline V & 5 & 2,332 & 30,000 \\
\hline W & 5 & N/A & 16,950 \\
\hline X & 3.4 & 2,717 & 9,093 \\
\hline Y & 3 & 1,240 & 18,887 \\
\hline Z & 3 & 1,311 & 2,480 \\
\hline AA & 2.9 & 2,521 & 23,847 \\
\hline BB & 2 & 958 & 6,000 \\
\hline CC & 1.4 & N/A & N/A \\
\hline DD & 1 & N/A & 19,000 \\
\hline EE & 0.9 & N/A & 3,000 \\
\hline
\end{tabular}

Table 118 Enrolment cohort size, with the identified Service FTE and the Occasions of Service as provided by 31 responders (institutions).

\begin{tabular}{|c|c|c|}
\hline Enrolment cohort size & Service FTE & Occasions of service \\
\hline 67,000 & 12 & N/A \\
\hline 66,928 & 12.6 & 8,500 \\
\hline 63,406 & 18 & $10,779 * *$ \\
\hline 60,000 & 11.8 & $8,952 * * *$ \\
\hline 52,331 & 8.8 & 4,861 \\
\hline 51,338 & 10 & $10,844 *$ \\
\hline 44,208 & 7 & 5,152 \\
\hline 40,000 & 13.4 & 6,839 \\
\hline 38,000 & 13 & $\sim 7,000$ \\
\hline 37,531 & 12.4 & $\mathrm{~N} / \mathrm{A}$ \\
\hline 36,746 & 11.2 & N/A \\
\hline 31,114 & 5 & 2,561 \\
\hline 30,767 & 8 & 5,492 \\
\hline 30,000 & 5 & 2,332 \\
\hline 27,000 & $5.2+1.2$ locums in peak & 6,081 \\
\hline 26,975 & 7.9 & 3,532 \\
\hline 24,000 & 6.8 & 5,589 \\
\hline 23,847 & 2.9 & 2,521 \\
\hline 23,109 & 7.4 & 5,601 \\
\hline 23,000 & 10 & 6,800 \\
\hline 22,736 & 7.9 & 7,640 \\
\hline 19,000 & 1 & N/A \\
\hline 18,887 & 3 & 1,240 \\
\hline 16,950 & 5 & N/A \\
\hline 12,000 & 5.1 & 2,457 \\
\hline 9,093 & 3.4 & 2,717 \\
\hline 6,000 & 2 & 958 \\
\hline
\end{tabular}




\begin{tabular}{|c|c|c|}
\hline Enrolment cohort size & Service FTE & Occasions of service \\
\hline 3,000 & 0.9 & N/A \\
\hline 2,480 & 3 & 1,311 \\
\hline N/A & 10 & N/A \\
\hline N/A & 1.4 & N/A \\
\hline
\end{tabular}

Q116 Average occasions of service in 2017

Data was provided by 22 of the 32 responding institutions.

Range varied from 1.6 to 4.9 occasions of service. For twenty (20) institutions the average number of occasions of service sat within the range of 2-4 occasions of service. There were only two outliners to this range. One institution reported an average of 4.9 and another bookended at the other extreme with 1.6 occasions of service.

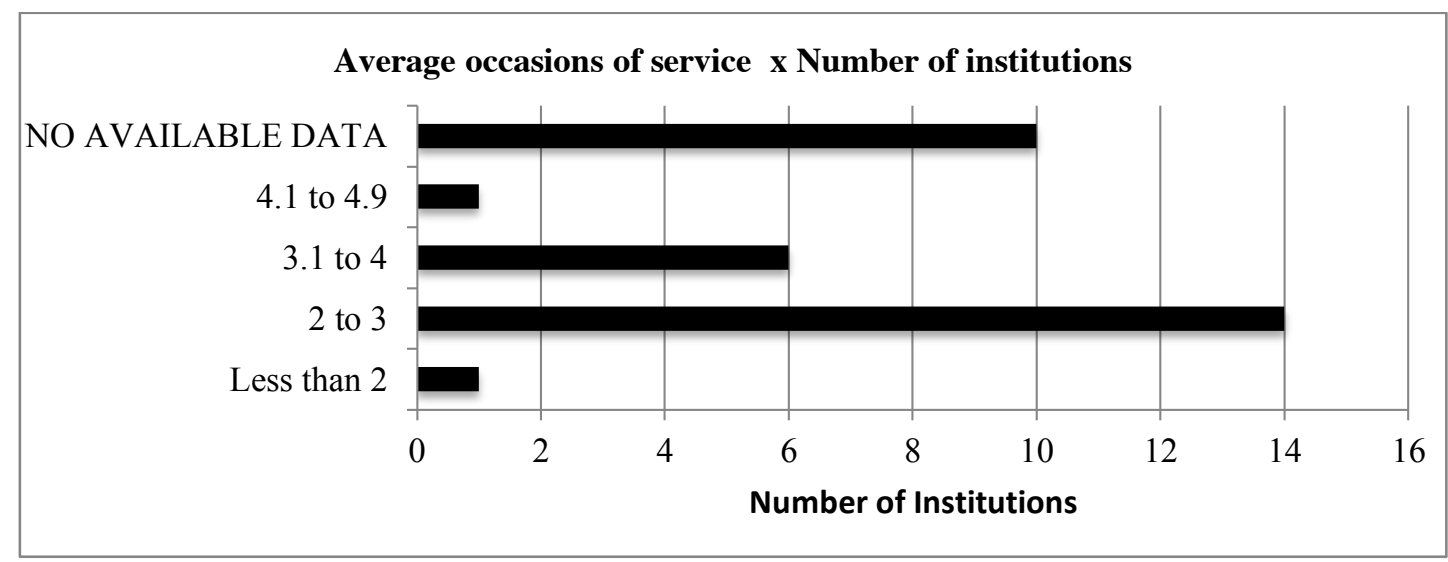

Figure 26

Q117 What was the percentage of 'no shows' for counselling appointments during 2017?

The range of responses for the percentage of 'no shows' for counselling appointments during 2017 was 2 to $21.4 \%$. Specific responses from 16 institutions show that the most common percentage of appointments considered 'no show' was $8 \%(\mathrm{~N}=5)$. (See Table 119.) Nine (9) responses reported 'no show' percentages of 6\%-10\%. Four (4) responses indicated 'no shows' to $5 \%$ or less. Fifteen (15) responses of 'no available data' were received. (See Figure 26.)

Table 119

\begin{tabular}{|c|c|}
\hline \multicolumn{2}{|c|}{ No Shows to 1-1 counselling appointments } \\
\hline Number of institutions & $\begin{array}{c}\text { Percentage of appointments that were "No } \\
\text { Shows" }\end{array}$ \\
\hline 1 & $2 \%$ \\
\hline 1 & $3 \%$ \\
\hline 2 & $5 \%$ \\
\hline 1 & $7.2 \%$ \\
\hline 5 & $8 \%$ \\
\hline 2 & $9 \%$ \\
\hline 1 & $10 \%$ \\
\hline 1 & $11.1 \%$ \\
\hline 1 & $14 \%$ \\
\hline 1 & $21.4 \%$ \\
\hline
\end{tabular}




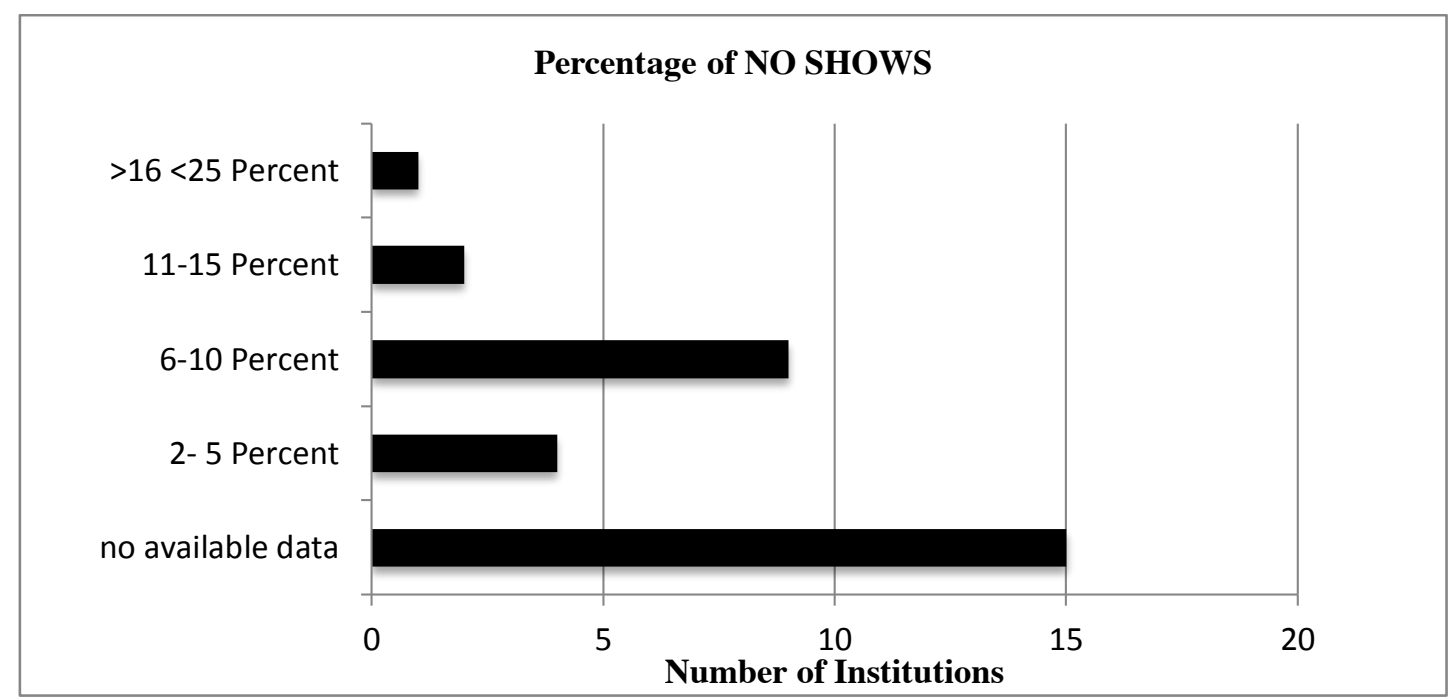

Figure 27

Q118 Percentage of students presenting for a single consult.

Nineteen (19) institutions indicated 'no available data' for the percentage of students presenting for a single consult. The reported single consult percentage range was $7.5-67 \%$.

Specific responses from 12 institutions show seven (7) responses falling within $35 \%$ to $50 \%$ for single consults.

Table 120

\begin{tabular}{|c|c|}
\hline \multicolumn{2}{|c|}{ Single consultations } \\
\hline Number of responses & Percentage of single consults \\
\hline 1 & $7.5 \%$ \\
\hline 1 & $10 \%$ \\
\hline 1 & $19.7 \%$ \\
\hline 3 & $35 \%$ \\
\hline 1 & $38.2 \%$ \\
\hline 1 & $42 \%$ \\
\hline 1 & $45 \%$ \\
\hline 1 & $50 \%$ \\
\hline 1 & $57 \%$ \\
\hline 1 & $67 \%$ \\
\hline
\end{tabular}

Q119 Which five of the following were within the most prevalent presenting issues identified by service clients during 2017? Tick five only.

Thirty-two (32) responses were received for the five (5) most prevalent presenting issues identified by service clients during 2017. Table 116 provides the detail of all the responses.

The five (5) most identified presenting issues in order of prevalence were:

- Stress

- Mental ill-health: acute or chronic

- Relationship issues

- Academic progress

- Low mood 
Table 121 - The top 5 presenting issues appear in italics.

\begin{tabular}{|l|c|}
\hline \multicolumn{1}{|c|}{ Presenting Issues - 2017 } & Percentage of Institutions \\
\hline Stress & $\mathbf{9 6 . 8 8 \%}$ \\
\hline $\begin{array}{l}\text { Mental ill-health: acute or chronic } \\
\text { (DSM diagnostic category includes depression } \\
\text { and anxiety) }\end{array}$ & $\mathbf{8 1 . 2 5 \%}$ \\
\hline Relationship issues & $\mathbf{7 1 . 8 8 \%}$ \\
\hline Academic Progress & $\mathbf{6 8 . 7 5 \%}$ \\
\hline Low mood & $\mathbf{6 8 . 7 5 \%}$ \\
\hline Avoidance issues (procrastination) & $25.00 \%$ \\
\hline Adjustment issues & $25.00 \%$ \\
\hline Other (please specify) & $21.88 \%$ \\
\hline Performance anxiety & $21.88 \%$ \\
\hline Loss of motivation & $18.75 \%$ \\
\hline $\begin{array}{l}\text { Welfare needs (financial hardship, } \\
\text { accommodation/homelessness etc) }\end{array}$ & $6.25 \%$ \\
\hline Administrative issues & $0.00 \%$ \\
\hline
\end{tabular}

Q120 Issues regularly identified by counsellors in their assessment of students who attend for counselling/psychological services

Thirty-two (32) responses were received and issues identified by counsellors in their assessment of students are shown in Figure 28.

Concerns with $93-55 \%$ selection:

- Social anxiety - social isolation

- Perfectionism - negatively impacting on academic achievement

- Panic attack

- Interpersonal conflict

- Performance anxiety

- Inadequate interpersonal skills to establish significant friendships

- Too little personal motivation to support academic progression

- Asperger's Syndrome/Autism Spectrum disorder

- Personality Disorder

- Sexuality issues

- Domestic violence

Concerns with $50-44 \%$ selection:

- Sexual assault/rape

- Gender identity issues

- Inadequate skills to be a successful independent learner

- Harassment/Stalking behaviour

- Unclear career goals

Concerns with less than $40 \%$ selection:

- Psychosis 
- Inadequate skills to support independent living

- Other (please specify)

- Paranoia

- Unplanned pregnancy

- Gambling problems

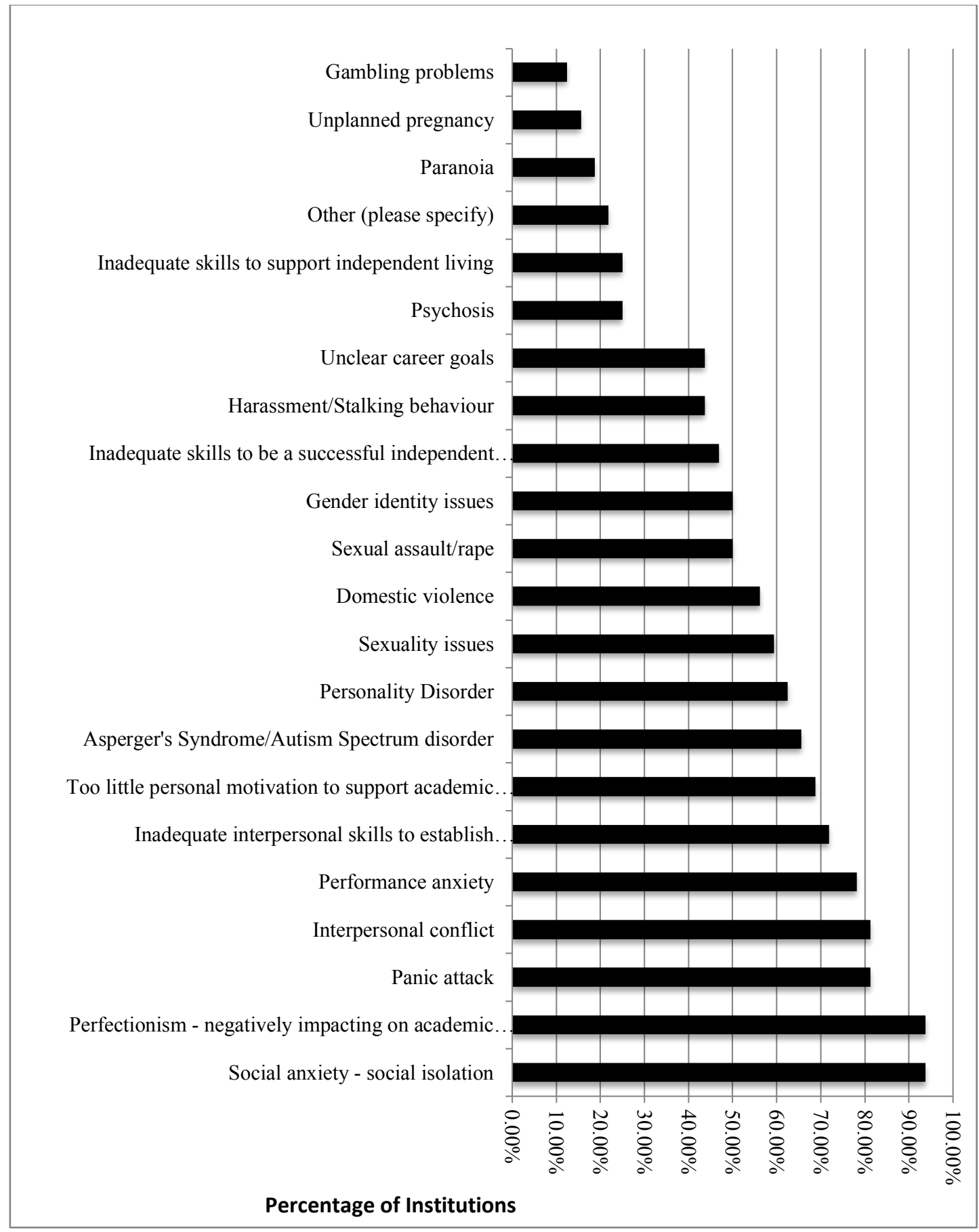

Figure 28

Q121 What percentage of service clients were referred as per the following categories?

Referred to community mental health team for urgent assessment

Nine (9) responses were received. The range of responses was $2-15 \%$. 
Four (4) responses were 5\% or below and four (4) responses were between $10 \%-15 \%$.

Table 122

\begin{tabular}{|c|c|}
\hline \multicolumn{2}{|c|}{ Referred to community mental health team for urgent assessment } \\
\hline Number of responses & Percentage of referrals \\
\hline 1 & $2 \%$ \\
\hline 2 & $3 \%$ \\
\hline 1 & $5 \%$ \\
\hline 2 & $7 \%$ \\
\hline 2 & $10 \%$ \\
\hline
\end{tabular}

Referred to hospital casualty/emergency service

Seven (7) responses were received. The response range was $1-10 \%$.

Five (5) responses were for $2 \%$ or less and two (2) responses were received for $10 \%$.

Table 123

\begin{tabular}{|c|c|}
\hline \multicolumn{2}{|c|}{ Referred to hospital casualty/emergency service } \\
\hline Number of responses & Percentage of referrals \\
\hline 3 & $1 \%$ \\
\hline 2 & $2 \%$ \\
\hline 2 & $10 \%$ \\
\hline
\end{tabular}

Referred to GP for services provided via government funded health services or private health insurance at first screening/assessment

Nine (9) responses were received. The response range was 1-25\%. Four (4) responses were for 2\% or less. Three (3) responses were received for $10 \%$ or higher.

Table 124

\begin{tabular}{|c|c|}
\hline $\begin{array}{c}\text { Referred to GP for services provided via government funded health services } \\
\text { or private health insurance at first screening/assessment }\end{array}$ \\
\hline Number of responses & Percentage of referrals \\
\hline 2 & $1 \%$ \\
\hline 2 & $2 \%$ \\
\hline 1 & $10 \%$ \\
\hline 1 & $20 \%$ \\
\hline 1 & $25 \%$ \\
\hline
\end{tabular}

Referred to GP for services provided via government funded health services or private health insurance after service session limit reached

Four (4) responses were received. The response range was 4-20\%. One (1) response was received for $4 \%$. Two (2) responses were received for $15 \%$ and one (1) for $20 \%$.

Table 125

Referred to GP for services provided via government funded health services or private health insurance after service session limit reached

\begin{tabular}{|c|c|}
\hline Number of responses & Percentage of referrals \\
\hline 1 & $4 \%$ \\
\hline 2 & $15 \%$ \\
\hline 1 & $20 \%$ \\
\hline
\end{tabular}

Referred to GP for services provided via government funded health services or private health insurance because of need for specific expertise for ongoing treatment

Seven (7) responses were received. The response range was 1-30\%. 
Five (5) responses were 5\% or less. One (1) response was received for each of $15 \%$ and $30 \%$.

Table 126

Referred to GP for services provided via government funded health services or private health insurance because of need for specific expertise for on going treatment

\begin{tabular}{|c|c|}
\hline Number of responses & Percentage of referrals \\
\hline 1 & $1 \%$ \\
\hline 1 & $4 \%$ \\
\hline 3 & $5 \%$ \\
\hline 1 & $15 \%$ \\
\hline 1 & $30 \%$ \\
\hline
\end{tabular}

Referred to GP for referral to psychiatric services (government funded or private health insurance)

Six (6) responses were received. The response range was 1-30\%. A single response (1) was received for each of the following referral percentages: $1 \%, 3 \%, 5 \%, 6 \%, 10 \%$, and $30 \%$.

Table 127

\begin{tabular}{|c|c|}
\hline $\begin{array}{c}\text { Referred to GP for referral to psychiatric services (government funded or } \\
\text { private health insurance) }\end{array}$ \\
\hline Number of responses & Percentage of referrals \\
\hline 1 & $1 \%$ \\
\hline 1 & $3 \%$ \\
\hline 1 & $5 \%$ \\
\hline 1 & $6 \%$ \\
\hline 1 & $10 \%$ \\
\hline
\end{tabular}

Referred to GP for referral to other allied health service provider

Five (5) responses were received. The response range was $1-5 \%$. Two (2) responses were for $1 \%$ and $5 \%$ and one (1) for $3 \%$.

Table 128

\begin{tabular}{|c|c|}
\hline \multicolumn{2}{|c|}{ Referred to GP for referral to other allied health service provider } \\
\hline Number of responses & Percentage of referrals \\
\hline 2 & $1 \%$ \\
\hline 1 & $3 \%$ \\
\hline 2 & $5 \%$ \\
\hline
\end{tabular}

\section{Referred directly to psychologist in private practice}

Six (6) responses were received. The response range was $2-40 \%$. Two (2) responses were received for $5 \%$ and a single (1) response for each of the following percentages: $2 \%, 15 \%, 20 \%$ and $40 \%$.

Table 129

\begin{tabular}{|c|c|}
\hline \multicolumn{2}{|c|}{ Referred directly to psychologist in private practice } \\
\hline Number of responses & Percentage of referrals \\
\hline 1 & $2 \%$ \\
\hline 2 & $5 \%$ \\
\hline 1 & $15 \%$ \\
\hline 1 & $20 \%$ \\
\hline 1 & $40 \%$ \\
\hline
\end{tabular}




\section{Referred directly to other allied health service provider}

Six (6) responses were received. The response range was $1-10 \%$.

Two (2) responses were received for $2 \%$ and a single response for each of the following percentages: $1 \%, 4 \%, 5 \%$, and $10 \%$.

Table 130

\begin{tabular}{|c|c|}
\hline \multicolumn{2}{|c|}{ Referred directly to other allied health service provider } \\
\hline Number of responses & Percentage of referrals \\
\hline 1 & $1 \%$ \\
\hline 2 & $2 \%$ \\
\hline 1 & $4 \%$ \\
\hline 1 & $5 \%$ \\
\hline 1 & $10 \%$ \\
\hline
\end{tabular}

\section{Referred to university based psychology school clinic}

Three (3) responses were received. The response range was 1-20\%. A single response was received for each of the following percentages: $1 \%, 8 \%$ and $20 \%$.

Table 131

\begin{tabular}{|c|c|}
\hline \multicolumn{2}{|c|}{ Referred directly to other allied health service provider } \\
\hline Number of responses & Percentage of referrals \\
\hline 1 & $1 \%$ \\
\hline 1 & $8 \%$ \\
\hline 1 & $20 \%$ \\
\hline
\end{tabular}

\section{Referred to other area of university (e.g. academic advisor, Course coordinator, student advisory centre)}

Twelve responses reported $0 \%$. Eight (8) responses were received for percentages above $0 \%$. The response range was $0-20 \%$. A single response was received for each of the following percentages: $1 \%, 8 \%$ and $20 \%$.

Table 132

\begin{tabular}{|c|c|}
\hline \multicolumn{2}{|c|}{ Referred to other area of university } \\
\hline Number of responses & Percentage of referrals \\
\hline 12 & $0 \%$ \\
\hline 1 & $1 \%$ \\
\hline 1 & $10 \%$ \\
\hline 3 & $20 \%$ \\
\hline 1 & $23 \%$ \\
\hline 1 & $30 \%$ \\
\hline 1 & $60 \%$ \\
\hline
\end{tabular}

Q122 What percentage of students who accessed counselling and psychological services at your institution in 2017 were in need of urgent attention because of concern about personal safety or the safety of others (suicidal intent, domestic violence, stalker, psychosis etc)?

Thirty (30) responses were received and sixteen (16) of those responses provided data. Six (6) responses indicated that $5-10 \%$ of the students who accessed counselling and psychological services in 2017 were in need of urgent attention because of concern about personal safety or the safety of others. Four (4) responses indicated a percentage of less than 5\% and three (3) responses identified $11-20 \%$ of the students, who accessed counselling and psychological services in 2017 , were in need of urgent attention because of concern about personal safety or the safety of others. 


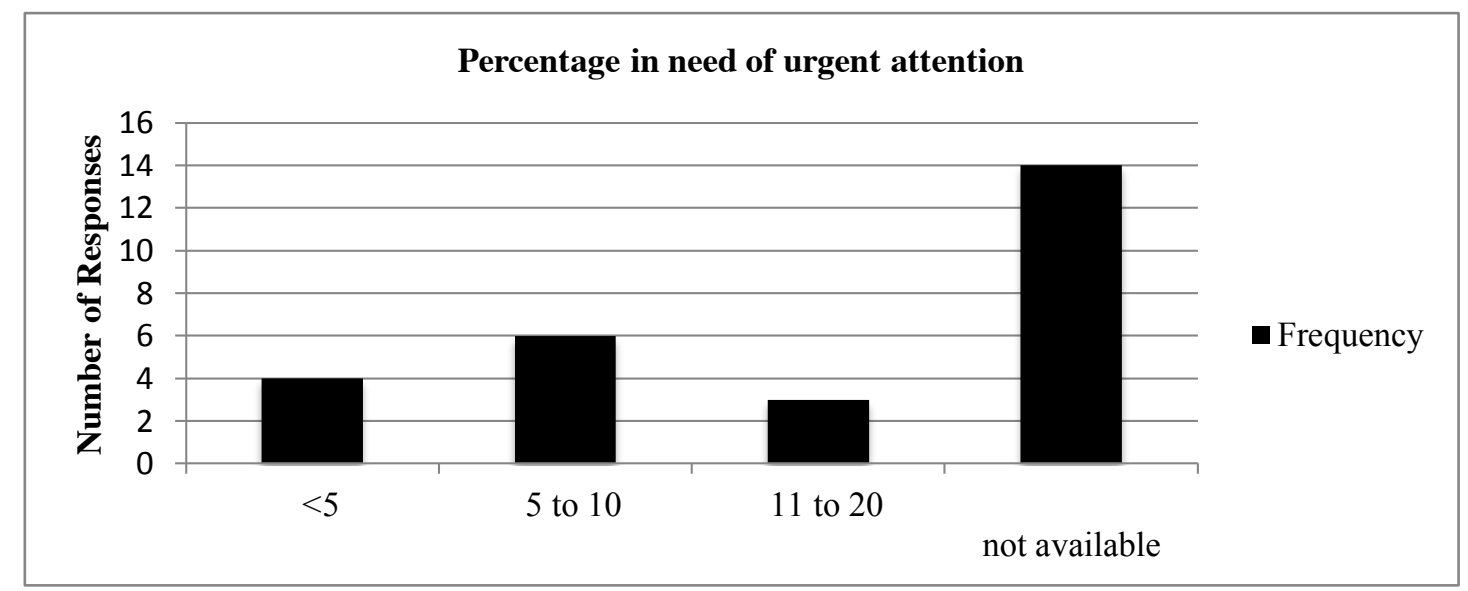

Figure 29

Q123 In 2017, which of the following factors were identified as significant contributors to the level of psychological distress in those that presented for urgent attention?

Table 133

\begin{tabular}{|c|c|c|}
\hline Factors contributing to students' psychological distress & $\begin{array}{l}\text { Number of } \\
\text { responses }\end{array}$ & $\begin{array}{l}\text { Percentage of } \\
\text { responses }\end{array}$ \\
\hline Depression & 31 & $96.88 \%$ \\
\hline Anxiety & 28 & $87.50 \%$ \\
\hline Academic issues & 23 & $71.88 \%$ \\
\hline Personality disorder & 22 & $68.75 \%$ \\
\hline Victim of violence/sexual assault/rape & 22 & $68.75 \%$ \\
\hline $\begin{array}{l}\text { University related issues (course complaints, research supervisor } \\
\text { complaints, inappropriate relationship with uni staff member etc) }\end{array}$ & 22 & $68.75 \%$ \\
\hline Unexpected illness or death of family member or close friend & 22 & $68.75 \%$ \\
\hline Relationship issues & 20 & $62.50 \%$ \\
\hline Psychosis (emerging or acute or low level and chronic) & 17 & $53.13 \%$ \\
\hline Bipolar disorder & 15 & $46.88 \%$ \\
\hline Social isolation & 14 & $43.75 \%$ \\
\hline Behaviour in class or with other students & 12 & $37.50 \%$ \\
\hline Childhood abuse & 8 & $25.00 \%$ \\
\hline Finances & 8 & $25.00 \%$ \\
\hline Homelessness & 8 & $25.00 \%$ \\
\hline Unexpected events in home region (natural disaster, civil unrest, war) & 8 & $25.00 \%$ \\
\hline Childhood sexual assault & 6 & $18.75 \%$ \\
\hline Legal concerns & 5 & $15.63 \%$ \\
\hline Physical health issues & 5 & $15.63 \%$ \\
\hline Unexpected pregnancy & 4 & $12.50 \%$ \\
\hline Other (please specify) & 0 & $0.00 \%$ \\
\hline
\end{tabular}

The top 10 presenting issues across all the responding institutions follow in rank order:

Issues rated by more than $70 \%$ of responders in rank order:

- Depression,

- Anxiety

- Academic issues

Issues rated by $65-70 \%$ of responders all equally rated (68.75\%):

- Personality Disorder 
- Victim of violence/sexual assault/rape

- University related issues

- Unexpected illness or death of family member or close friend

Issues rated by $45-64 \%$ of responders in rank order:

- Relationship issues

- Psychosis

- Bipolar disorder

Q124 Does your institution have any way of knowing how many students enrolled at the institution died by suicide in 2017 ?

Less than $22 \%$ of responding institutions had a way of knowing how many students died by suicide in 2017.

\begin{tabular}{|c|c|c|}
\hline $\begin{array}{l}\text { Institution knows how many students died by } \\
\text { suicide in } 2017\end{array}$ & $\begin{array}{l}\text { Number of } \\
\text { responses }\end{array}$ & $\begin{array}{l}\text { Percentage of } \\
\text { responses }\end{array}$ \\
\hline Don't know & 13 & $40.63 \%$ \\
\hline No & 12 & $37.50 \%$ \\
\hline Yes & 7 & $21.88 \%$ \\
\hline $\begin{array}{l}\text { If Yes, please give the known number of students who } \\
\text { died by suicide. }\end{array}$ & 7 & \\
\hline
\end{tabular}

Seven (7) institutions provided data on the number of student deaths by suicide in 2017 :

One (1) institution reported 7 student deaths by suicide.

One (1) institution reported 5 student deaths by suicide.

Two (2) institutions reported 2 student deaths by suicide.

Three (3) institutions reported 1 student death by suicide.

Q125 Do you know of any students who were clients of your service died by suicide in 2017?

Eleven 11) managers (34.4\%) responded that they knew of students who were clients of their service who died by suicide in 2017 . The majority of managers $(65.6 \%)$ responded that they did not know of any students who were clients of their service died by suicide in 2017 .

\begin{tabular}{|c|c|c|}
\hline \multicolumn{3}{|c|}{$\begin{array}{l}\text { Do you know of any students who were clients of your service died by } \\
\text { suicide in 2017? }\end{array}$} \\
\hline Answer Choices & $\begin{array}{l}\text { Percentage of } \\
\text { Responses }\end{array}$ & $\begin{array}{l}\text { Number of } \\
\text { Responses }\end{array}$ \\
\hline No & $65.63 \%$ & 21 \\
\hline Yes & $34.38 \%$ & 11 \\
\hline $\begin{array}{l}\text { If Yes, please give the known } \\
\text { number of students that were a client } \\
\text { of the service and who died by } \\
\text { suicide. }\end{array}$ & & 11 \\
\hline
\end{tabular}

Eleven (11) responses elaborated as requested on the number of students who were clients of the service who died by suicide in 2017. Seven (7) responses recorded that one (1) student who had been a client of the service died by suicide in 2017. One (1) response was recorded for zero (0) and one (1) response was received for both N/A and 'none were clients (past or current)'. 
Table 136

\begin{tabular}{|c|c|}
\hline Number of students who were clients of your service died by suicide in 2017 & $\begin{array}{c}\text { Number of } \\
\text { Responses }\end{array}$ \\
\hline 0 & 1 \\
\hline 1 & 7 \\
\hline 2 & 1 \\
\hline N/A & 1 \\
\hline None were clients (past or current) & 1 \\
\hline
\end{tabular}

Q126 During 2017 did your service collect data on the number of service clients who acknowledge past or current suicide gestures or attempts?

The majority of institutions did not collect data on past or current suicide gestures or attempts from presenting students.

Table 137

\begin{tabular}{|l|c|c|}
\hline $\begin{array}{l}\text { Institution collected data (2017) on students who } \\
\text { acknowledged past or current suicide gestures or attempts }\end{array}$ & $\begin{array}{c}\text { Number of } \\
\text { responses }\end{array}$ & $\begin{array}{c}\text { Percentage of } \\
\text { responses }\end{array}$ \\
\hline No & 24 & $75.00 \%$ \\
\hline Yes & 8 & $25.00 \%$ \\
\hline $\begin{array}{l}\text { If YES please specify the known number (not the percent) of } \\
\text { students who identified a history of suicide gestures or } \\
\text { attempts }\end{array}$ & 6 & \\
\hline
\end{tabular}

Six (6) comments added by respondents providing additional data on the number of students who identified a history of suicide gestures or attempts in 2017.

Comments:

- 95 and this relates to current, not historical so the number would be higher

- 34

- 987

- Approx 120 - only reported if it was presenting problem

- 625

- 42

Q127 What percentage of service clients during 2017 were assessed by the counsellors as being 'at risk' for self-harm or suicide?

Eighteen (18) institutions reported that data was not available.

Nine (9) institutions provided data. Four (4) responses reported 2-5\% of students were assessed by the counsellors as being 'at risk' for self-harm or suicide. Two (2) responses identified $>5-7 \%$, and there were single responses (1) for $10 \%, 14 \%$ and $20 \%$.

Table 138

\begin{tabular}{|} 
Table 138 \\
\begin{tabular}{|c|c|}
\hline \multicolumn{1}{|c|}{ Service clients assessed by the counsellors as being 'at risk' for self-harm or } \\
suicide in $\mathbf{2 0 1 7}$
\end{tabular} \\
\hline Number of Responses & Percentage assessed as at risk \\
\hline 18 & Data not available \\
\hline 1 & $20 \%$ \\
\hline 1 & $14 \%$ \\
\hline 1 & $10 \%$ \\
\hline 2 & $>5$ to $7 \%$ \\
\hline 4 & 2 to $5 \%$ \\
\hline
\end{tabular}


Q128 During 2017 did your service collect data on the number of students who presented with alcohol or other drug overuse or abuse behaviours?

The majority of responses $(\mathrm{N}=20)$ did not collect data on Alcohol and Other Drugs (AOD) use. Twelve (12) responses reported that AOD data was collected.

Table 139

\begin{tabular}{|l|c|c|}
\hline \multicolumn{1}{|c|}{ Collected data on AOD use } & $\begin{array}{c}\text { Percentage of } \\
\text { responses }\end{array}$ & $\begin{array}{c}\text { Number of } \\
\text { responses }\end{array}$ \\
\hline No & $62.50 \%$ & 20 \\
\hline Yes & $37.50 \%$ & 12 \\
\hline $\begin{array}{l}\text { If Yes, please give the known number (not percent) of } \\
\text { students who were understood to be overusing or } \\
\text { abusing alcohol or other drugs. }\end{array}$ & & \\
\hline
\end{tabular}

Of the twelve (12) institutions that reported collecting information on AOD use, eight (8) institutions provided further data on the number of presenting students that reported AOD use. The range of responses varied from 0.16 to 345 . Specific responses were: $0.16,0.27,1,16,17,26,100$ 120 and 345.

Q129 During 2017 did your service collect data on the number of students attending your service who presented with self-harming behaviour (cutting, burning, selfflagellation etc)?

The majority $(\mathrm{N}=22)$ reported not collecting data on self-harming behaviour.

Table 140

\begin{tabular}{|l|c|c|}
\hline \multicolumn{1}{|c|}{ Collected data on self-harming behaviour } & $\begin{array}{c}\text { Percentage of } \\
\text { responses }\end{array}$ & $\begin{array}{c}\text { Number of } \\
\text { responses }\end{array}$ \\
\hline No & $68.75 \%$ & 22 \\
\hline Yes & $31.25 \%$ & 10 \\
\hline $\begin{array}{l}\text { If Yes, please give the known number (not percent) } \\
\text { of students who were understood to be self-harming. }\end{array}$ & & 9
\end{tabular}

Nine (9) of the ten (10) managers that reported collecting data on self-harming behaviour provided further information. Seven (7) managers reporting on self-harming behaviour also provided further data on the 'known number' (not percentage). The range of responses varied from $\mathrm{N}=0.9$ to $\mathrm{N}=180$. The specific responses were: 0.9, 1, 2, 8, approx 60, 76 and 180 .

Q130 During 2017 did your service collect data on the number of students who presented to the service with internet overuse or online gaming problems?

Over $90 \%$ of managers reported that data on internet overuse was not collect. Only three (3) responses were in the affirmative. Only two (2) provided further detail. One (1) reported that one (1) student was recorded as presenting with internet overuse issues. Another reported 0 presentations.

Q131 During 2017 did your service collect data on the number of students who presented to the service with eating disorders or disturbed eating?

Seventy-three percent of responses indicated that they did not collect data on the number of students presenting with eating disorders or disturbed eating. Of those collecting data $(\mathrm{N}=8)$, six (6) managers provided data on the number of students presenting with eating disorders or disturbed eating. The range was 1 to 100. Single responses were reported for: 1, 2, 3, 32 and two (2) responses indicated 100 students presented with eating disorders or disturbed eating.

Q132 During 2017 did your service model of practice encourage professional staff to apply a formal diagnosis as part of the assessment or treatment process?

Seventy-five (75) percent of managers indicated that there was no firm practice of giving formal diagnosis and that recording a diagnosis was left to the discretion of the professional and the 
context of the professional relationship and treatment needs or disability provision needs. Twentyfive (25) percent of responses indicated that formal diagnosis is not part of the assessment and treatment process because of the concern about the impact of the diagnostic label on student future career or insurance options etc.

Table 141

\begin{tabular}{|l|c|c|}
\hline \multicolumn{1}{|c|}{ Formal diagnosis applied? } & $\begin{array}{c}\text { Percentage of } \\
\text { responses }\end{array}$ & $\begin{array}{c}\text { Number of } \\
\text { responses }\end{array}$ \\
\hline $\begin{array}{l}\text { No - formal diagnosis is not part of the assessment and } \\
\text { treatment process because of the concern about the impact of } \\
\text { the diagnostic label on student future career or insurance } \\
\text { options etc. }\end{array}$ & $25 \%$ & 8 \\
\hline $\begin{array}{l}\text { No firm practice of giving formal diagnosis - left to the } \\
\text { discretion of the professional and the context of the } \\
\text { professional relationship and treatment needs or disability } \\
\text { provision needs. }\end{array}$ & & \\
\hline
\end{tabular}

Six (6) respondents skipped this question.

Q133 During 2017, what percentage of service clients had mental health presentations that would have warranted a diagnosis using DSM 5 or ICD-10 criteria?

Only four (4) institutions provided a percentage response. Specific responses provided were: $25 \%$, $60 \%$ (x 2) and another stated that anecdotally $75 \%$ would have warranted a diagnosis using DSM 5 or ICD-10 criteria.

Q134 During 2017 what percentage of students presenting to the service had impairment so severe that your professional staff would have encouraged enrolment withdrawal for a medical leave period?

Only six (6) institutions were able to draw on data for this question. Responses ranged between less than $1 \%$ to $23 \%$. Specific responses provided were: less than $1 \%, 2 \%, 3 \%, 5 \%, 10 \%$ and $23 \%$.

Q135 During 2017, what percentage of students presenting to the service were so impaired that they could only continue with their enrolment with regular (at least fortnightly) on-going support/treatment?

Only five (5) institutions were able to draw on data. All responses were 5\% or less. Specific responses provided were: $<1 \%, 3 \%, 4 \%$ and $5 \%(\mathrm{x} 2)$.

Q136 In 2017, what percentage of the clients attending the service were hospitalised for treatment of mental illness episodes or for containment of self harm or suicide intent?

Only six (6) institutions were able to draw on data. All responses were less than 10\%. The percentages provided were: $0.5 \%, 2 \%, 4 \%, 5 \%$ (x 2), and $6 \%$.

Q137 In 2017, what percentage of clients attending the service had mental health concerns and severe stress due to academic progress concerns (academic suspension/exclusion, excessive academic performance expectations, academic failure, fear of failure, negative impact of perfectionism, performance anxiety etc?

Only five (5) institutions were able to draw on data. The responses were $9.5 \%, 15 \%, 20 \%, 34 \%$ and $60 \%$.

Q138 Was there an increase in demand for counselling in 2017 compared to 2016.

More than $60 \%$ of institutions reported an increase in demand for counselling in 2017 compared to 2016. The percentage increase varied from less than or equal to $5 \%$ to greater than $20 \%$. 


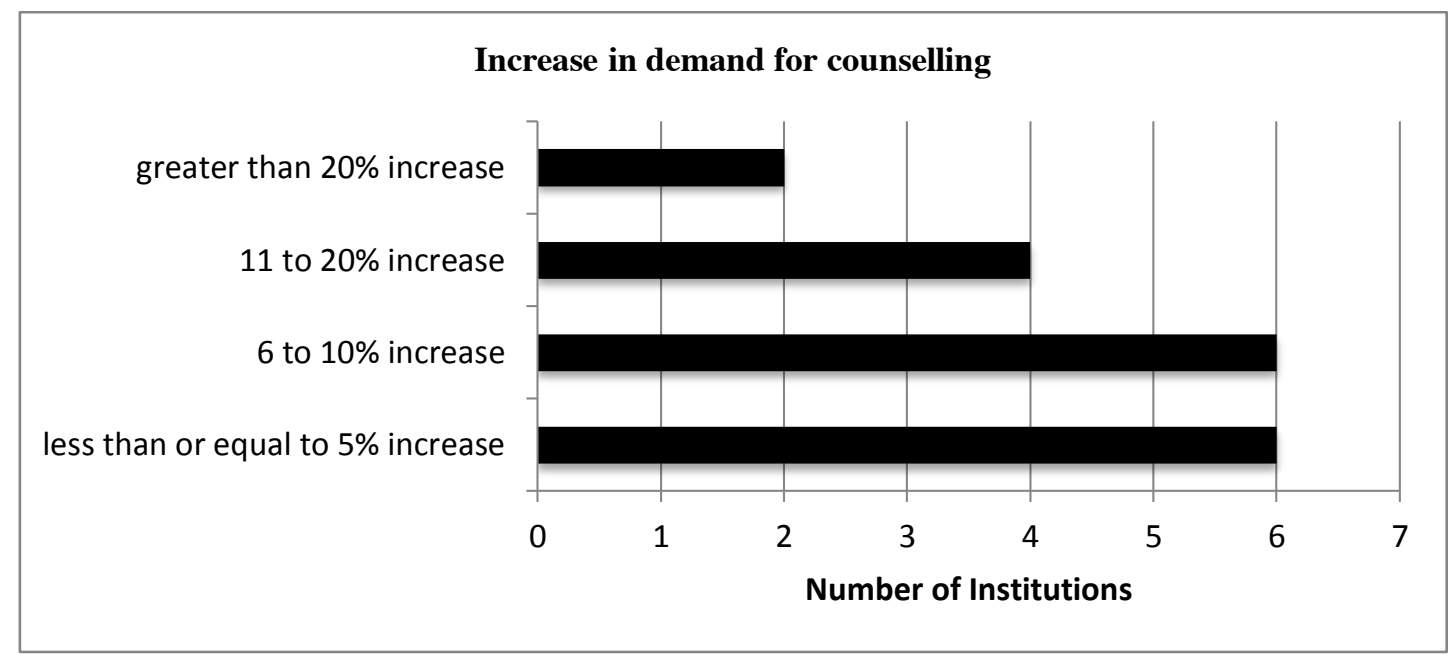

Figure 30

\section{Q139 Students No Show to workshops}

Responses reported variation from more than $50 \%$ to approximately $5 \%$ of 'student no shows' following registration for a workshop.

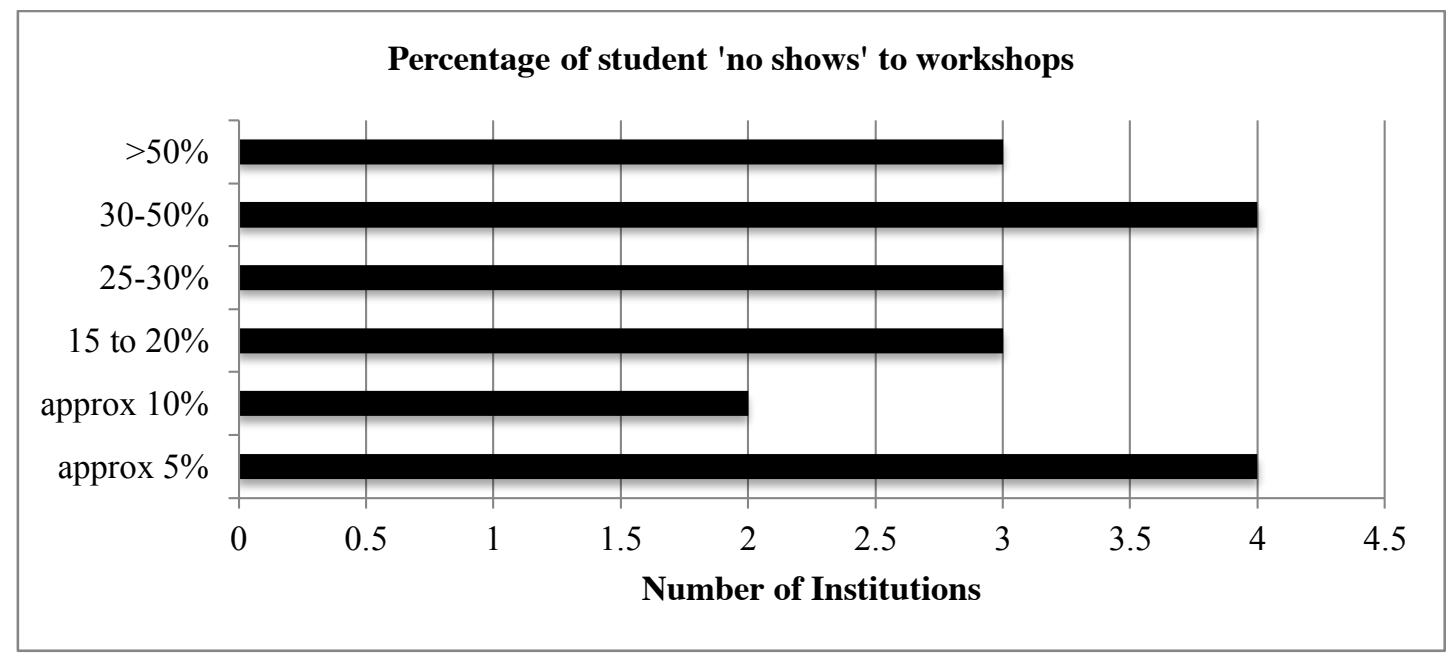

Figure 31

Q140 In the USA, Canada, Australia and New Zealand heads of counselling services have been reporting over the last decade a steady increase in the complexity and severity of student mental health presentations along with an increase in the proportion of students affected. Indicate level of agreement with this statement.

All of the thirty-two (32) responses agreed or strongly agreed with the statement. Eighteen managers $(56.25 \%)$ agreed and indicated that service data provided some support for the statement: 'In the USA, Canada, Australia and New Zealand heads of counselling services have been reporting over the last decade a steady increase in the complexity and severity of student mental health presentations along with an increase in the proportion of students affected.' Six (6) managers, $(18.75 \%)$, 'strongly agreed' and indicated that 'multiple sources of hard data' supported the statement. Others $(6.25 \%)$ agreed and indicated that there was 'considerable hard data sampled across a number of services'. A further six (6) managers (18.75\%) agreed but had no hard data to support the claim. 
Table 142

\begin{tabular}{|l|c|c|}
\hline \multicolumn{1}{|c|}{ Answer choices } & $\begin{array}{c}\text { Percentage } \\
\text { of responses }\end{array}$ & $\begin{array}{c}\text { Number of } \\
\text { responses }\end{array}$ \\
\hline $\begin{array}{l}\text { Do not agree - students have always presented with serious and complex issues. } \\
\text { Reported changes are due to other factors such as societal awareness of mental } \\
\text { health/illness and increased staff expertise leading to more appropriate } \\
\text { diagnosis and treatment plans. }\end{array}$ & & \\
\hline $\begin{array}{l}\text { Agree but no hard data- Professional staff report a greater proportion of } \\
\text { caseload with complex presentation and significant mental health issues. NO } \\
\text { hard data for this beyond professional staff reports. }\end{array}$ & 18.75\% & 6 \\
\hline $\begin{array}{l}\text { Agree - some support provided by service data - Our service data reflects } \\
\text { increasing severity and complexity of presentation by students attending the } \\
\text { service. Increases in the proportion of students with more severe and complex } \\
\text { mental health issues cannot be validated due to limits imposed by service } \\
\text { resources. }\end{array}$ & & \\
\hline $\begin{array}{l}\text { Agree - considerable hard data sampled across a number of services - Our } \\
\text { service data reflects increasing severity and complexity of presentation by } \\
\text { students attending the service. Increases in the proportion of students with more } \\
\text { severe and complex mental health issues is also indicated based on data from } \\
\text { counselling services, disability services and health services available to our } \\
\text { students. }\end{array}$ & $56.25 \%$ & 18 \\
\hline $\begin{array}{l}\text { Strongly agree - multiple sources of hard data - Institution wide student 'well- } \\
\text { being' surveys and mental health awareness programs indicate that mental } \\
\text { health concerns within the student population is significant and possibly at } \\
\text { proportions higher than expected in the general population. Our service data } \\
\text { reflects increasing severity and complexity in student presentations. Increases } \\
\text { in the proportion of students with more severe and complex mental health } \\
\text { issues is also indicated based on presentations to counselling services, disability } \\
\text { services and health services available to students. }\end{array}$ & & \\
\hline
\end{tabular}

Increase in complexity and severity student mental health presentations

Strongly agree - multiple data sources

Agree - considerable data

Agree - some service data

Agree - no hard data

Do not agree

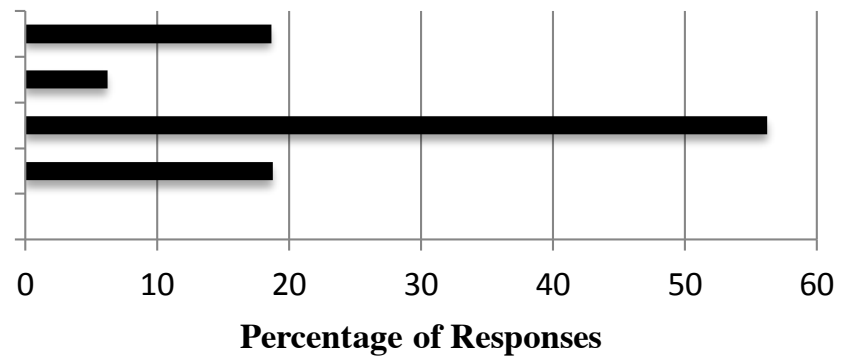

Figure 32

Q141 Cohort characteristics of those attending counselling services

Very scant data for cohort characteristics other than gender.

More females than males utilise counselling services. The only exception was for an institution reporting a $65 \%$ male enrolment. Figure 32 shows the reported gender differences for nineteen (19) institutions linked to managers who were able to provide this data for the survey. 


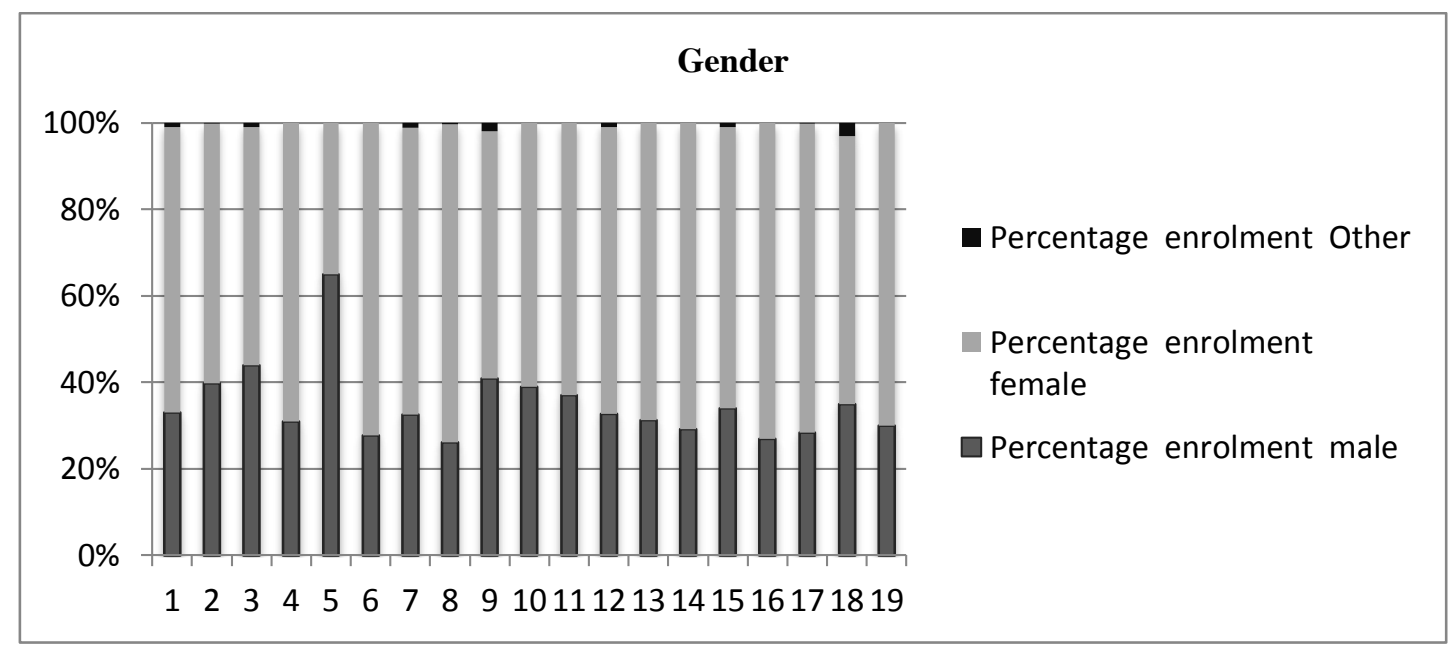

Figure 33

$Q 142$ Value of completing the survey to heads of service

The heads of service valued the benchmarking survey. The survey is considered useful as: a benchmarking tool; assisting with preparation for the annual report; and for identifying data to be gathered in the coming year. Feedback identified an omission in the survey questions concerning the gathering of cohort enrolment data. Feedback also indicated that the survey could be shortened and it was suggested that any questions that gathered similar be identified and eliminated. Some of the requested data was difficult or impossible for the manager to access.

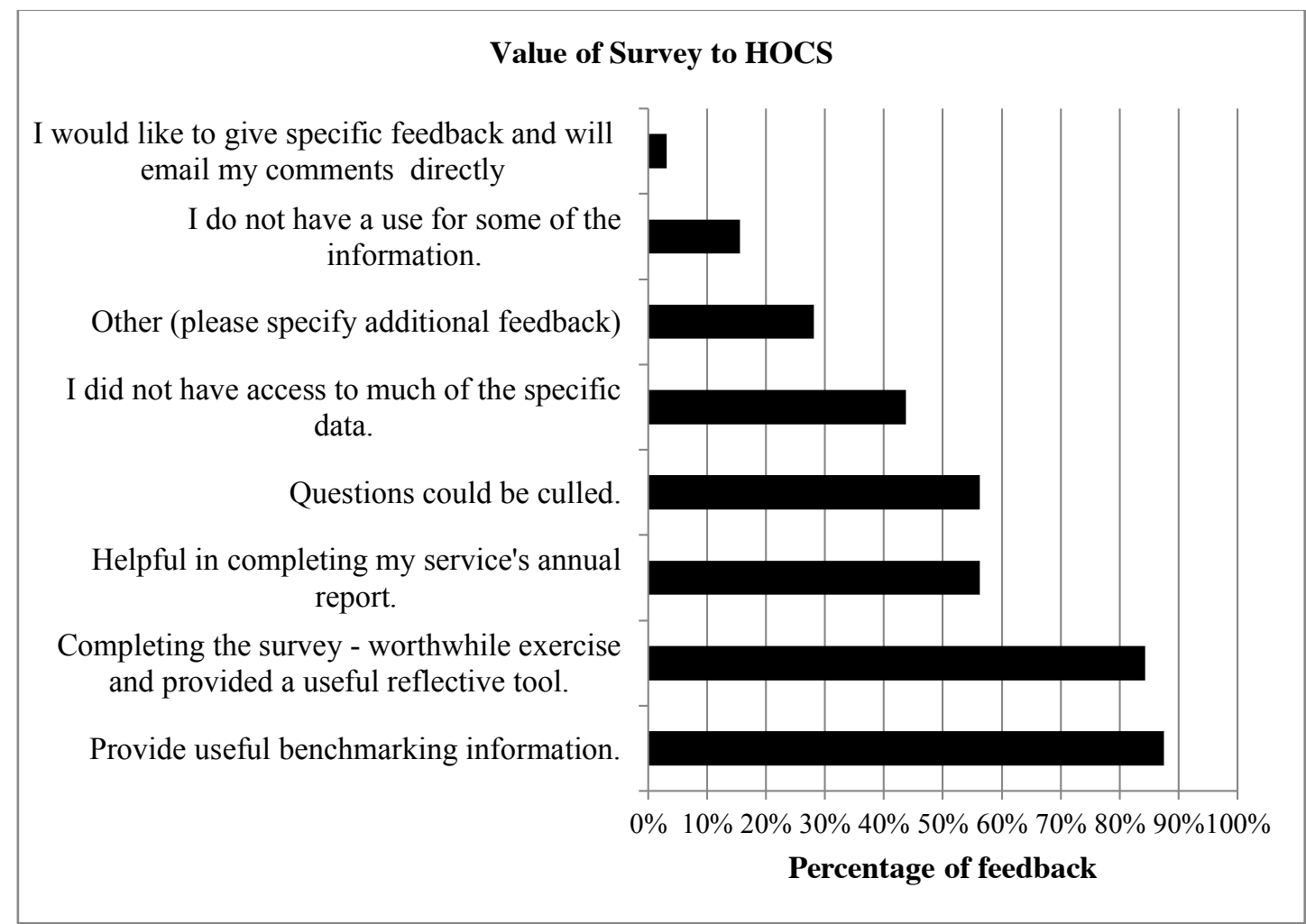

Figure 34

Specific feedback is copied below. Only detail (e.g. campus locations) that could be used to identify the respondent has been removed.

- Thanks. Be good to get the report :) 
- Yes too long, but pointed out gaps in our data collection, as the questions are in much greater depth than we collect. Often our data was aggregated - so not able to answer without an accompanying comment - therefore N/A was a frequent response.

- Also, requesting stats from the university as a whole, meant that it was aggregated across the 3 campuses, but if compared to our staffing - it would look woefully inadequate. That's why!

- Finally, an ability to go to a particular page number would be helpful, when going back later to provide some data!

- The survey was very long and time consuming trying to chase up information that we do not have access to within the counselling service.

- Some of the instructions did not seem to work, e.g. 'N/A' was rejected sometimes despite the instruction so I had to go back and re-do the answers with zeros.

- Also question on telephone or VOIP service did not offer an option to tick both.

- This has been a valuable exercise and provided me with insights and ideas about additional data our service can be collecting.

- Thank you for putting together - it has made me look at some of the current date we are collecting.

- Also in terms of headcount enrolments there was not space for including enabling programs (we have a headcount of 379 in preparatory programs) or non-award (we have a headcount of 1194) mainly in study abroad programs.

- Very helpful in identifying areas we need to work on.

- Some questions were repeated. The survey did not ask for Part-time undergraduate coursework total FTE. It also did not ask for Part-time postgraduate coursework.

- We offer Skype and phone counselling, but I could only click one or the other.

- $\quad$ Some sections would not proceed if N/A was typed into the box, so I had to leave it blank (i.e. did not want non-numerical data).

- The survey made me realise how much we do not get to do in our Service.

- Some issues with submission - stopped at question 115 , would only allow me to submit 0 Value, not N/A so this slowed me down considerably. 


\section{References:}

Andrews, A., (2016) ANZSSA Heads of Counselling Services Benchmarking Survey 2013 Summary Report. Journal of the Australian and New Zealand Student Services Association: Number 47, April 2016, 96-104.

International Association of Counselling Services Inc. (IACS) (n.d.) IACS Statement regarding recommended staff to student ratios. Retrieved February 2019 from http://www.iacsinc.org/staff-to-student-ratios.html

International Association of Counseling Services, Inc. (IACS) (n.d.) Standards for University and College Counseling Services. Revised October 10, 2010; Amended November 8, 2014; Sec. IV. Amended October 22, 2016. Retrieved February 2019 from https://0201.nccdn.net/4_2/000/000/053/0e8/2017-STANDARDS-10-5-17.pdf

The Association for University and College Counseling Centre Directors (AUCCCD) (n.d.) The Association for University and College Counseling Center Directors Annual Survey: Reporting period: September 1, 2015 through August 31, 2016. Retrieved from

https://www.aucccd.org/assets/documents/aucccd\%202016\%20monograph\%20-\%20public.pdf

The author may be contacted:

Annie Andrews (previously Director, UNSW Sydney Counselling and Psychological Services) is a JANZSSA editor and can be contacted at janzssa@gmail.com

\section{Please cite this paper as:}

Andrews, A. (2019) ANZSSA Heads of Counselling Services HOCS Benchmarking Survey: 2018 Summary Report Journal of the Australian and New Zealand Student Services Association, 27(1), 67-171.

doi.org.10.30688/janzssa.2019.06 